\title{
Predicting the sparticle spectrum from GUTs via SUSY threshold corrections with SusyTC
}

\author{
Stefan Antusch ${ }^{a, b}$ and Constantin Sluka ${ }^{a}$ \\ ${ }^{a}$ Department of Physics, University of Basel, \\ Klingelbergstr. 82, CH-4056 Basel, Switzerland \\ ${ }^{b}$ Max-Planck-Institut für Physik (Werner-Heisenberg-Institut), \\ Föhringer Ring 6, D-80805 München, Germany \\ E-mail: Stefan.Antusch@unibas.ch, Constantin.Sluka@unibas.ch
}

ABstract: Grand Unified Theories (GUTs) can feature predictions for the ratios of quark and lepton Yukawa couplings at high energy, which can be tested with the increasingly precise results for the fermion masses, given at low energies. To perform such tests, the renormalization group (RG) running has to be performed with sufficient accuracy. In supersymmetric (SUSY) theories, the one-loop threshold corrections (TC) are of particular importance and, since they affect the quark-lepton mass relations, link a given GUT flavour model to the sparticle spectrum. To accurately study such predictions, we extend and generalize various formulas in the literature which are needed for a precision analysis of SUSY flavour GUT models. We introduce the new software tool SusyTC, a major extension to the Mathematica package REAP [1], where these formulas are implemented. SusyTC extends the functionality of REAP by a full inclusion of the (complex) MSSM SUSY sector and a careful calculation of the one-loop SUSY threshold corrections for the full down-type quark, up-type quark and charged lepton Yukawa coupling matrices in the electroweak-unbroken phase. Among other useful features, SusyTC calculates the one-loop corrected pole mass of the charged (or the CP-odd) Higgs boson as well as provides output in SLHA conventions, i.e. the necessary input for external software, e.g. for performing a two-loop Higgs mass calculation. We apply SusyTC to study the predictions for the parameters of the CMSSM (mSUGRA) SUSY scenario from the set of GUT scale Yukawa relations $\frac{y_{e}}{y_{d}}=-\frac{1}{2}, \frac{y_{\mu}}{y_{s}}=6$, and $\frac{y_{\tau}}{y_{b}}=-\frac{3}{2}$, which has been proposed recently in the context of SUSY GUT flavour models.

Keywords: Beyond Standard Model, GUT, Renormalization Group, Supersymmetric Standard Model

ARXIV EPRINT: 1512.06727 


\section{Contents}

1 Introduction $\quad 1$

2 Predictions for Yukawa coupling ratios from GUTs 3

3 SUSY threshold corrections \& numerical procedure $\quad 5$

4 The REAP extension SusyTC 16

5 The sparticle spectrum predicted from CG factors $\quad 17$

$\begin{array}{ll}5.1 \text { Comments and discussion } & 20\end{array}$

$6 \quad$ Summary and conclusion $\quad 22$

A The $\beta$-functions in the seesaw type-I extension of the MSSM 23

A.1 One-loop $\beta$-functions 23

$\begin{array}{lll}\text { A.2 } & \text { Two-loop } \beta \text {-functions } & 28\end{array}$

B Self-energies and one-loop tadpoles including inter-generational mixing 36

C SusyTC documentation $\quad 42$

\section{Introduction}

Supersymmetric (SUSY) Grand Unified Theory (GUT) models of flavour are promising candidates towards solving the open questions of the Standard Model (SM) of Particle Physics. They embrace the unification of the SM gauge couplings, a dark matter candidate, a solution to the gauge hierarchy problem and an explanation of the hierarchies of masses and mixing angles in the flavour sector. Whether a flavour GUT model can successfully explain the observations in the flavour sector, depends on the renormalization group (RG) evolution of the Yukawa matrices from the GUT scale to lower energies. Furthermore, it is known that $\tan \beta$ enhanced supersymmetric threshold corrections (see e.g. [2-6]) are essential in the investigation of mass (or Yukawa coupling) ratios predicted at the GUT scale. Interesting well-known GUT predictions in this context are $b-\tau$ and $b-\tau-t$ unification (for early work see e.g. [7-10]) and $y_{\mu}=3 y_{s}[11] .{ }^{1}$ Other promising quarklepton mass relations at the GUT scale have been discussed in $[12,13]$, e.g. $y_{\tau}= \pm \frac{3}{2} y_{b}$, $y_{\mu}=6 y_{s}$ or $y_{\mu}=\frac{9}{2} y_{s}$. Various aspects regarding the impact of such GUT relations for phenomenology have been studied in the literature, see e.g. [14-31] for recent works.

\footnotetext{
${ }^{1} y_{i}$ are the Yukawa couplings in the diagonal basis.
} 
With the discovery of the Higgs boson at the LHC $[32,33]$ and the possible discovery of sparticles in the near future, the question whether a set of SUSY soft-breaking parameters can be in agreement with both, specific SUSY threshold corrections as required for realizing the flavour structure of a GUT model, and constraints from the Higgs boson mass and results on the sparticle spectrum, gains importance. To accurately study this question, we introduce the new software tool SusyTC, a major extension to the Mathematica package REAP [1].

REAP, which is designed to run the SM and neutrino parameters in seesaw scenarios with a proper treatment of the right-handed neutrino thresholds, is a convenient tool for top-down analyses of flavour (GUT) models, with the advantage of a user-friendly Wolfram Mathematica front-end. However, the SUSY sector is not included. In order to take supersymmetric threshold corrections into account in the analyses, for example of the $A_{4}$ flavour GUT models in [34,35], the following procedure was undertaken: first REAP was used to run the Yukawa matrices in the MSSM from the GUT-scale to a user-defined "SUSY"-scale. At this scale, the SUSY threshold corrections were incorporated as mere model parameters, in a simplified treatment assuming, e.g., degenerate first and second generation sparticle masses (cf. [36]), without specializing any details on the SUSY sector. Finally, the Yukawa matrices, corrected by these $\tan \beta$-enhanced thresholds, were taken as input for a second run of REAP, evolving the parameters in the SM from the "SUSY"scale to the top-mass scale. ${ }^{2}$ Although this procedure is quite SUSY model-independent, it only allows to study the constraints on the SUSY sector indirectly (i.e. via the introduced additional parameters and with simplifying assumptions), and it is unclear whether an explicit SUSY scenario with these assumptions and requirements can be realised.

The aim of this work is to make full use of the SUSY threshold corrections to gain information on the SUSY model parameters from GUTs. Towards this goal we extend and generalize various formulas in the literature which are needed for a precision analysis of SUSY flavour GUT models and implement them in SusyTC. For example, SusyTC includes the full (CP violating) MSSM SUSY sector, sparticle spectrum calculation, a careful calculation of the one-loop SUSY threshold corrections for the full down-type quark, up-type quark and charged lepton Yukawa coupling matrices in the electroweak-unbroken phase, and automatically performs the matching of the MSSM to the SM, including $\overline{\mathrm{DR}}$ to $\overline{\mathrm{MS}}$ conversion. Among other useful features, SusyTC calculates the one-loop corrected pole mass of the charged (or the CP-odd) Higgs boson as well as provides output in SLHA conventions, i.e. the necessary input for external software, e.g. for performing a two-loop Higgs mass calculation.

SusyTC is specifically developed to perform top-down analyses of SUSY flavour GUT models. This is a major difference to other well-known SUSY spectrum generators (e.g. [3741], see e.g. [42] for a comparison), which run experimental constraints from low energies to high energies, apply GUT-scale boundary conditions, run back to low energies and repeat this procedure iteratively. SusyTC instead starts directly from the GUT-scale, allowing the

\footnotetext{
${ }^{2}$ Such a treatment of threshold corrections as additional model parameters is now implemented in the latest version of RGEMSSMON.m of REAP 11.1.2.
} 
user to define general (complex) Yukawa, trilinear, and soft-breaking matrices, as well as non-universal gaugino masses, as input. These parameters are then run to low energies, thereby enabling an investigation whether the GUT-scale Yukawa matrix structures of a given SUSY flavour GUT model are in agreement with experimental data.

We apply SusyTC to study the predictions for the parameters of the Constrained MSSM (mSUGRA) SUSY scenario from the GUT-scale Yukawa relations $\frac{y_{e}}{y_{d}}=-\frac{1}{2}, \frac{y_{\mu}}{y_{s}}=6$, and $\frac{y_{\tau}}{y_{b}}=-\frac{3}{2}$, which have been proposed recently in the context of SUSY GUT flavour models. With a Markov Chain Monte Carlo analysis we find a "best-fit" benchmark point as well as the $1 \sigma$ ranges for the sparticle masses and the correlations between the SUSY parameters. Without applying any constraints from LHC SUSY searches or dark matter, we find that the considered GUT scenario predicts a CMSSM sparticle spectrum above past LHC sensitivities, but within reach of the current LHC run or a future high-luminosity upgrade. Furthermore, the scenario generically features a bino-like neutralino LSP and a stop NLSP with a mass that can be close to the present bounds.

This paper is organized as follows: in section 2 we review GUT predictions for Yukawa coupling ratios. In section 3, we describe the numerical procedure in SusyTC and present the main used formulas. We give a short introduction to the new features SusyTC adds to REAP in section 4 . In section 5 we study the predictions for the parameters of the CMSSM SUSY scenario from the above mentioned GUT-scale Yukawa relations with SusyTC. In the appendices we present other relevant formulas and a detailed documentation of SusyTC.

\section{Predictions for Yukawa coupling ratios from GUTs}

GUTs not only contain a unification of the SM forces, they also unify fermions into joint representations. After the GUT gauge group is broken to the SM gauge group, this can lead to predictions for the ratios of down-type quark and charged lepton Yukawa couplings which result from group theoretical Clebsch Gordan (CG) factors. To confront such predictions of GUT models with the experimental data, the RG evolution of the Yukawa couplings from high to low energies has to be performed, including (SUSY) threshold corrections.

In SU(5) GUTs, for example, the right-handed down-type quarks and the lepton doublets are unified in five-dimensional representations of $\mathrm{SU}(5)$ and the quark doublets plus right-handed up-type quarks and right-handed charged leptons are unified in a tendimensional SU(5) representation. The Higgs doublets are supplemented by $\mathrm{SU}(3)_{c}$ triplets and embedded into five-dimensional representations of SU(5). Using only these fields and a single renormalizable operator to generate the Yukawa couplings for the down-type quarks and charged leptons, so-called minimal SU(5) predicts $Y_{e}=Y_{d}^{T}$ for the Yukawa matrices at the GUT scale. To correct this experimentally challenged scenario, SU(5) GUT flavour models often introduce a 45-dimensional Higgs representation, which can lead to the Georgi-Jarlskog relations $y_{\mu}=-3 y_{s}$ and $y_{e}=\frac{1}{3} y_{d}[11]$.

It was pointed out in [12] (see also [13]) that other promising Yukawa coupling GUT ratios can emerge in $\mathrm{SU}(5)$, e.g. $y_{\tau}= \pm \frac{3}{2} y_{b}, y_{\mu}=6 y_{s}$ or $y_{\mu}=\frac{9}{2} y_{s}$, and $y_{e}=-\frac{1}{2} y_{d}$ from higher dimensional GUT operators containing for instance a GUT breaking 24-dimensional Higgs representation. A convenient test whether GUT predictions for the first two families 
can be consistent with the experimental data is provided by the - RG invariant and SUSY threshold correction invariant ${ }^{3}$ - double ratio [36]

$$
\left|\frac{y_{\mu}}{y_{s}} \frac{y_{d}}{y_{e}}\right|=10.7_{-0.8}^{+1.8}
$$

While the Georgi-Jarlskog relations [11] imply a double ratio of 9, disfavoured by more than $2 \sigma$, other combinations of CG factors $[12,13]$, e.g. $y_{\mu}=6 y_{s}$ and $y_{e}=-\frac{1}{2} y_{d}$ can be in better agreement (here with a double ratio of 12 , within the $1 \sigma$ experimental range).

The combination of GUT-scale Yukawa relations $y_{e}=-\frac{1}{2} y_{d}, y_{\mu}=6 y_{s}$, and $y_{\tau}=-\frac{3}{2} y_{b}$ (as direct result of CG factors, cf. table 1 and figure 1, or as approximate relation after diagonalization of the GUT-scale Yukawa matrices $Y_{d}$ and $Y_{e}$ ) has been used to construct $\mathrm{SU}(5)$ SUSY GUT flavour models in refs. [34, 35, 43, 44]. A subset of these relation, $y_{\mu}=6 y_{s}$, and $y_{\tau}=-\frac{3}{2} y_{b}$, has been used in [45]. In addition to providing viable quark and charged lepton masses, the GUT CG factors $\left(Y_{e}\right)_{j i} /\left(Y_{d}\right)_{i j}=-\frac{1}{2}$ and 6 can also be applied to realize the promising relation between the lepton mixing angle $\theta_{13}^{\mathrm{PMNS}}$ and the Cabibbo angle, $\theta_{13}^{\text {PMNS }} \simeq \theta_{C} \sin \theta_{23}^{\text {PMNS }}$, in flavour models, as discussed in [46].

As mentioned above, in supersymmetric GUT models the SUSY threshold corrections can have an important influence on the Yukawa coupling ratios. When the MSSM is matched to the SM, integrating out the sparticles at loop-level leads to the emergence of effective operators, which can contribute sizeably to the Yukawa couplings, depending on the values of the sparticle masses, $\tan \beta$, and the soft-breaking trilinear couplings. Thereby, via the SUSY threshold corrections, a given set of GUT predictions for the ratios $\frac{y_{\tau}}{y_{b}}, \frac{y_{\mu}}{y_{s}}$ and $\frac{y_{e}}{y_{d}}$ imposes important constraints on the SUSY spectrum.

The SUSY threshold corrections can be subdivided into three classes: while at treelevel the down-type quarks only couple to the Higgs field $H_{d}$, via exchange of sparticles at one-loop level they can also couple to $H_{u}$, as shown in figure 2 in section 3 . When the sparticles are integrated out the emerging effective operator is enhanced for large $\tan \beta$ (i.e. "tan $\beta$-enhanced"). Analogously, there are also $\tan \beta$-enhanced threshold corrections to the charged lepton Yukawa couplings. For $Y_{u}$, however, the threshold effects emerging from effective couplings to $H_{d}$ are tan $\beta$-suppressed. The second class of threshold corrections emerges from the supersymmetric loops shown in figure 3. While some of them are strongly suppressed, others lead to the emergence of effective operators proportional to the soft SUSY-breaking trilinear couplings. For large trilinear couplings, they too can become important. Finally there are threshold corrections from exchange of heavy Higgs doublets and canonical normalization of external fields (i.e. from wavefunction renormalization diagrams), as shown in figures 4 and 5-7, respectively. Given the importance of the SUSY threshold corrections, we will discuss them and their implementation in SusyTC in detail in the next section.

\footnotetext{
${ }^{3}$ The invariance under SUSY threhold corrections holds under some generic conditions, cf. [36].
} 


\begin{tabular}{|cccc|}
\hline$A B$ & $C D$ & $R$ & $\left(Y_{e}\right)_{j i} /\left(Y_{d}\right)_{i j}$ \\
\hline$H_{24} F$ & $T \bar{H}_{45}$ & $\overline{\mathbf{4 5}}$ & $-\frac{1}{2}$ \\
$H_{24} F$ & $T \bar{H}_{5}$ & $\overline{\mathbf{5}}$ & $-\frac{3}{2}$ \\
$H_{24} T$ & $F \bar{H}_{5}$ & $\mathbf{1 0}$ & 6 \\
\hline
\end{tabular}

Table 1. Examples for dimension 5 effective operators $(A B)_{R}(C D)_{\bar{R}}$ and CG factors emerging from the supergraphs of figure 1 when $R$ and $\bar{R}$ are integrated out [12].

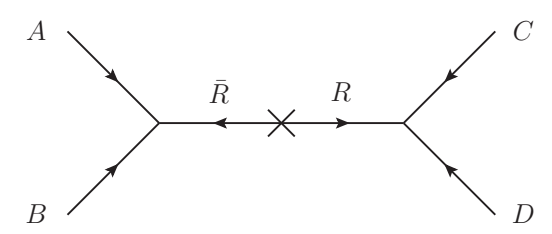

Figure 1. Supergraphs corresponding to the operators of table 1 generating effectively Yukawa couplings when the pair of messengers fields $R$ and $\bar{R}$ is integrated out.

\section{SUSY threshold corrections \& numerical procedure}

We follow the notation of REAP [1] (see also [47]) and use a RL convention for the Yukawa matrices. The MSSM superpotential extended by a type-I seesaw mechanism [48-54] is thus given by

$$
\begin{aligned}
W_{\mathrm{MSSM}}= & Y_{e_{i j}} E_{i}^{c} H_{d} \cdot L_{j}+Y_{\nu_{i j}} N_{i}^{c} H_{u} \cdot L_{j} \\
& +Y_{d_{i j}} D_{i}^{c} H_{d} \cdot Q_{j}-Y_{u_{i j}} U_{i}^{c} H_{u} \cdot Q_{j}+\frac{1}{2} M_{n_{i j}} N_{i}^{c} N_{j}^{c}+\mu H_{u} \cdot H_{d},
\end{aligned}
$$

where the left-chiral superfields $\Phi^{c}$ contain the charge conjugated fields $\psi^{\dagger}$ and $\tilde{\phi}_{R}^{*}$. We use the totally antisymmetric $\mathrm{SU}(2)$ tensor $\epsilon_{12}=-\epsilon_{21}=1$ for the product $\Phi \cdot \Psi \equiv \epsilon_{a b} \Phi^{a} \Psi^{b}$. The soft-breaking Lagrangian is given by

$$
\begin{aligned}
-\mathcal{L}_{\text {soft }}= & -\frac{1}{2} M_{a} \lambda^{a} \lambda^{a}+\text { h.c. } \\
& +T_{e_{i j}} \tilde{e}_{R_{i}}^{*} H_{d} \cdot \tilde{L}_{j}+T_{\nu_{i j}} \tilde{\nu}_{R_{i}}^{*} H_{u} \cdot \tilde{L}_{j}+T_{d_{i j}} \tilde{d}_{R_{i}}^{*} H_{d} \cdot \tilde{Q}_{j}-T_{u_{i j}} \tilde{u}_{R_{i}}^{*} H_{u} \cdot \tilde{Q}_{j}+h . c . \\
& +\tilde{Q}_{i}^{\dagger} m_{\tilde{Q}_{i j}}^{2} \tilde{Q}_{j}+\tilde{L}_{i}^{\dagger} m_{\tilde{L}_{i j}}^{2} \tilde{L}_{j}+\tilde{u}_{R_{i}}^{*} m_{\tilde{u}_{i j}}^{2} \tilde{u}_{R_{j}}+\tilde{d}_{R_{i}}^{*} m_{\tilde{d}_{i j}}^{2} \tilde{d}_{R_{j}}+\tilde{e}_{R_{i}}^{*} m_{\tilde{e}_{i j}}^{2} \tilde{e}_{R_{j}}+\tilde{\nu}_{R_{i}}^{*} m_{\tilde{\nu}_{i j}}^{2} \tilde{\nu}_{R_{j}} \\
& +m_{H_{u}}^{2}\left|H_{u}\right|^{2}+m_{H_{d}}^{2}\left|H_{d}\right|^{2}+\left(m_{3}^{2} H_{u} \cdot H_{d}+h . c .\right) .
\end{aligned}
$$

Note that these conventions differ from SUSY Les Houches Accord 2 [55]. They can easily be translated by

\begin{tabular}{|c|ccccccccc|}
\hline REAP \& SusyTC & $Y_{u}$ & $Y_{d}$ & $Y_{e}$ & $T_{u}$ & $T_{d}$ & $T_{e}$ & $m_{\tilde{u}}^{2}$ & $m_{\tilde{d}}^{2}$ & $m_{\tilde{e}}^{2}$ \\
SLHA 2 & $Y_{u}^{T}$ & $Y_{d}^{T}$ & $Y_{e}^{T}$ & $T_{u}^{T}$ & $T_{d}^{T}$ & $T_{e}^{T}$ & $\left(m_{\tilde{u}}^{2}\right)^{T}$ & $\left(m_{\tilde{d}}^{2}\right)^{T}$ & $\left(m_{\tilde{e}}^{2}\right)^{T}$ \\
\hline
\end{tabular}

Since REAP includes the RG running in the type-I seesaw extension of the MSSM (with the $\overline{\mathrm{DR}}$ two-loop $\beta$-functions for the MSSM parameters and the neutrino mass operator given in [47]), we have calculated the $\overline{\mathrm{DR}}$ two-loop $\beta$-functions of the gaugino mass 
parameters $M_{a}$, the trilinear couplings $T_{f}$, the sfermion squared mass matrices $m_{\tilde{f}}^{2}$, and soft-breaking Higgs mass parameters $m_{H_{u}}^{2}$ and $m_{H_{d}}^{2}$ in the presence of $Y_{\nu}, M_{n}$ and $m_{\tilde{\nu}}^{2}$ (using the general formulas of [56]). We list these $\beta$-functions in appendix A.

The Yukawa matrices and soft-breaking parameters are evolved to the SUSY scale

$$
Q=\sqrt{m_{\tilde{t}_{1}} m_{\tilde{t}_{2}}},
$$

where the stop masses are defined by the up-type squark mass eigenstates $\tilde{u}_{i}$ with the largest mixing to $\tilde{t}_{1}$ and $\tilde{t}_{2} \cdot{ }^{4}$ REAP automatically integrates out the right-handed neutrinos, as described in [1]. We assume that $M_{n}$ is much larger than the SUSY scale $Q$. REAP also features the possibility to add one-loop right-handed neutrino thresholds for the SM parameters, following [57].

At the SUSY scale $Q$ the tree-level sparticle masses and mixings are calculated. Considering heavy sparticles and large $Q \gtrsim \mathrm{TeV}$ the SUSY threshold corrections are calculated in the electroweak (EW) unbroken phase. In the EW unbroken phase there are in total twelve types of loop diagrams contributing to the SUSY threshold corrections for $Y_{d}$ (cf. figures 2 and 3 ).

The SUSY threshold corrections to $Y_{d}$ are calculated in the basis of diagonal squark masses and are given by (in terms of $\overline{\mathrm{DR}}$ quantities)

$$
\tilde{Y}_{d}^{\mathrm{SM}}=P_{d} \tilde{Y}_{d}^{\prime} P_{Q}^{T} P_{h},
$$

where $P_{d}, P_{Q}$, and $P_{h}$ are the threshold corrections (3.12), (3.19), (3.20), and (3.22) due to canonical normalization. $\tilde{Y}_{d_{i j}}^{\prime}$ is given by

$$
\begin{aligned}
\tilde{Y}_{d_{i j}}^{\prime}= & \tilde{Y}_{d_{i j}}^{\mathrm{MSSM}} \cos \beta\left(1+\frac{1}{16 \pi^{2}} \tan \beta\left(\eta_{i j}^{G}+\eta_{i j}^{W}+\eta_{i j}^{B}+\eta_{i j}^{T}\right)+\frac{1}{16 \pi^{2}}\left(\epsilon_{i j}^{W}+\epsilon_{i j}^{B}+\epsilon_{i j}^{T}+\epsilon_{i j}^{H}\right)\right) \\
& +\tilde{T}_{d_{i j}}^{\mathrm{MSSM}} \cos \beta \frac{1}{16 \pi^{2}}\left(\zeta_{i j}^{G}+\zeta_{i j}^{B}\right),
\end{aligned}
$$

where

$$
\begin{aligned}
\eta_{i j}^{G} & =-\frac{8}{3} g_{3}^{2} \frac{\mu^{*}}{M_{3}} H_{2}\left(\frac{m_{\tilde{d}_{i}}^{2}}{\left|M_{3}\right|^{2}}, \frac{m_{\tilde{Q}_{j}}^{2}}{\left|M_{3}\right|^{2}}\right), \\
\eta_{i j}^{W} & =\frac{3}{2} g_{2}^{2} \frac{M_{2}^{*}}{\mu} H_{2}\left(\frac{M_{2}^{2}}{|\mu|^{2}}, \frac{m_{\tilde{Q}_{j}}^{2}}{|\mu|^{2}}\right), \\
\eta_{i j}^{B} & =\frac{3}{5} g_{1}^{2}\left(\frac{1}{9} \frac{\mu^{*}}{M_{1}} H_{2}\left(\frac{m_{\tilde{d}_{i}}^{2}}{\left|M_{1}\right|^{2}}, \frac{m_{\tilde{Q}_{j}}^{2}}{\left|M_{1}\right|^{2}}\right)+\frac{1}{3} \frac{M_{1}^{*}}{\mu} H_{2}\left(\frac{m_{\tilde{d}_{i}}^{2}}{|\mu|^{2}} \frac{\left|M_{1}\right|^{2}}{|\mu|^{2}}\right)+\frac{1}{6} \frac{M_{1}^{*}}{\mu} H_{2}\left(\frac{\left|M_{1}\right|^{2}}{|\mu|^{2}}, \frac{m_{\tilde{Q}_{j}}^{2}}{|\mu|^{2}}\right)\right), \\
\eta_{i j}^{T} & =-\frac{1}{\mu \tilde{Y}_{d_{i j}}} \sum_{n, m} \tilde{Y}_{d_{i m}} \tilde{T}_{u_{m n}}^{\dagger} \tilde{Y}_{u_{n j}} H_{2}\left(\frac{m_{\tilde{u}_{n}}^{2}}{|\mu|^{2}}, \frac{m_{\tilde{Q}_{m}}^{2}}{|\mu|^{2}}\right),
\end{aligned}
$$

\footnotetext{
${ }^{4}$ SusyTC can also be set to use the convention $Q=\sqrt{m_{\tilde{u}_{1}} m_{\tilde{u}_{6}}}$ or a user-defined SUSY scale, as described in appendix $\mathrm{C}$.
} 
correspond to the $\tan \beta$-enhanced loops of figure 2 , and

$$
\begin{aligned}
\epsilon_{i j}^{W} & =12 g_{2}^{2} C_{00}\left(\frac{|\mu|^{2}}{Q^{2}}, \frac{\left|M_{2}\right|^{2}}{|\mu|^{2}}, \frac{m_{\tilde{Q}_{j}}^{2}}{|\mu|^{2}}\right), \\
\epsilon_{i j}^{B} & =\frac{3}{5} g_{1}^{2}\left(\frac{4}{3} C_{00}\left(\frac{|\mu|^{2}}{Q^{2}}, \frac{\left|M_{1}\right|^{2}}{|\mu|^{2}}, \frac{m_{\tilde{d}_{i}}^{2}}{|\mu|^{2}}\right)+\frac{2}{3} C_{00}\left(\frac{|\mu|^{2}}{Q^{2}}, \frac{\left|M_{1}\right|^{2}}{|\mu|^{2}}, \frac{m_{\tilde{Q}_{j}}^{2}}{|\mu|^{2}}\right)\right), \\
\epsilon_{i j}^{T} & =\frac{1}{\tilde{Y}_{d_{i j}}} \sum_{n, m} \tilde{Y}_{d_{i m}} \tilde{Y}_{u_{m n}}^{\dagger} \tilde{Y}_{u_{n j}} H_{2}\left(\frac{m_{\tilde{u}_{n}}^{2}}{|\mu|^{2}}, \frac{m_{\tilde{Q}_{m}}^{2}}{|\mu|^{2}}\right), \\
\epsilon_{i j}^{H} & =\frac{2 \sin ^{2} \beta}{\tilde{Y}_{d_{i j}}} \sum_{n, m} \tilde{Y}_{d_{i m}} \tilde{Y}_{u_{m n}}^{\dagger} \tilde{Y}_{u_{n j}} B_{1}\left(\frac{m_{H}^{2}}{Q^{2}}\right), \\
\zeta_{i j}^{G} & =\frac{8}{3} g_{3}^{2} \frac{1}{M_{3}} H_{2}\left(\frac{m_{\tilde{d}_{i}}^{2}}{\left|M_{3}\right|^{2}}, \frac{m_{\tilde{Q}_{j}}^{2}}{\left|M_{3}\right|^{2}}\right), \\
\zeta_{i j}^{B} & =-\frac{3}{5} g_{1}^{2} \frac{1}{9} \frac{1}{M_{1}} H_{2}\left(\frac{m_{\tilde{d}_{i}}^{2}}{\left|M_{1}\right|^{2}}, \frac{m_{\tilde{Q}_{j}}^{2}}{\left|M_{1}\right|^{2}}\right),
\end{aligned}
$$

correspond to the loops in figures 3 and 4 , respectively, where the contributions $\zeta_{i j}^{G}$ and $\zeta_{i j}^{B}$ can become important in cases of small $\tan \beta$ and large trilinear couplings. The loop functions $H_{2}, C_{00}$, and $B_{1}$ are defined as

$$
\begin{aligned}
H_{2}(x, y) & \equiv \frac{x \log (x)}{(1-x)(x-y)}+\frac{y \log (y)}{(1-y)(y-x)}, \\
C_{00}(q, x, y) & \equiv \frac{1}{4}\left(\frac{3}{2}-\log (q)+\frac{x^{2} \log (x)}{(1-x)(x-y)}+\frac{y^{2} \log (y)}{(1-y)(y-x)}\right), \\
B_{1}(q) & \equiv-\frac{3}{4}+\frac{1}{2} \log q .
\end{aligned}
$$

$\tilde{Y}, \tilde{T}$ are the Yukawa- and trilinear coupling matrices rotated into the basis where the squark mass matrices are diagonal, using the transformations

$$
\begin{aligned}
Y_{u} & =\tilde{W}_{\tilde{u}} \tilde{Y}_{u} \tilde{W}_{\tilde{Q}}^{\dagger}, \\
T_{u} & =\tilde{W}_{\tilde{u}} \tilde{T}_{u} \tilde{W}_{\tilde{Q}}^{\dagger}, \\
m_{\tilde{Q}}^{2} & =\tilde{W}_{\tilde{Q}} m_{\tilde{Q}}^{2}{ }^{\operatorname{diag}} \tilde{W}_{\tilde{Q}}^{\dagger}, \\
m_{\tilde{u}}^{2} & =\tilde{W}_{\tilde{u}} m_{\tilde{u}}^{2 \operatorname{diag}} \tilde{W}_{\tilde{u}}^{\dagger},
\end{aligned}
$$

and analogously for down-type (s)quarks and charged (s)leptons.

The threshold correction $P_{h}$ due to canonical normalization of external Higgs doublets is given by

$$
P_{h}=\sqrt{\left(U \mathcal{K}_{h} U^{\dagger}\right)_{11}^{-1}}
$$

with $U$ defined by

$$
\left(\begin{array}{c}
h^{a} \\
H^{a}
\end{array}\right) \equiv U\left(\begin{array}{c}
-\epsilon_{a b} H_{d}^{a *} \\
H_{u}^{a}
\end{array}\right)=\left(\begin{array}{cc}
\cos \beta & \sin \beta \\
-\sin \beta & \cos \beta
\end{array}\right)\left(\begin{array}{c}
-\epsilon_{a b} H_{d}^{a *} \\
H_{u}^{a}
\end{array}\right),
$$



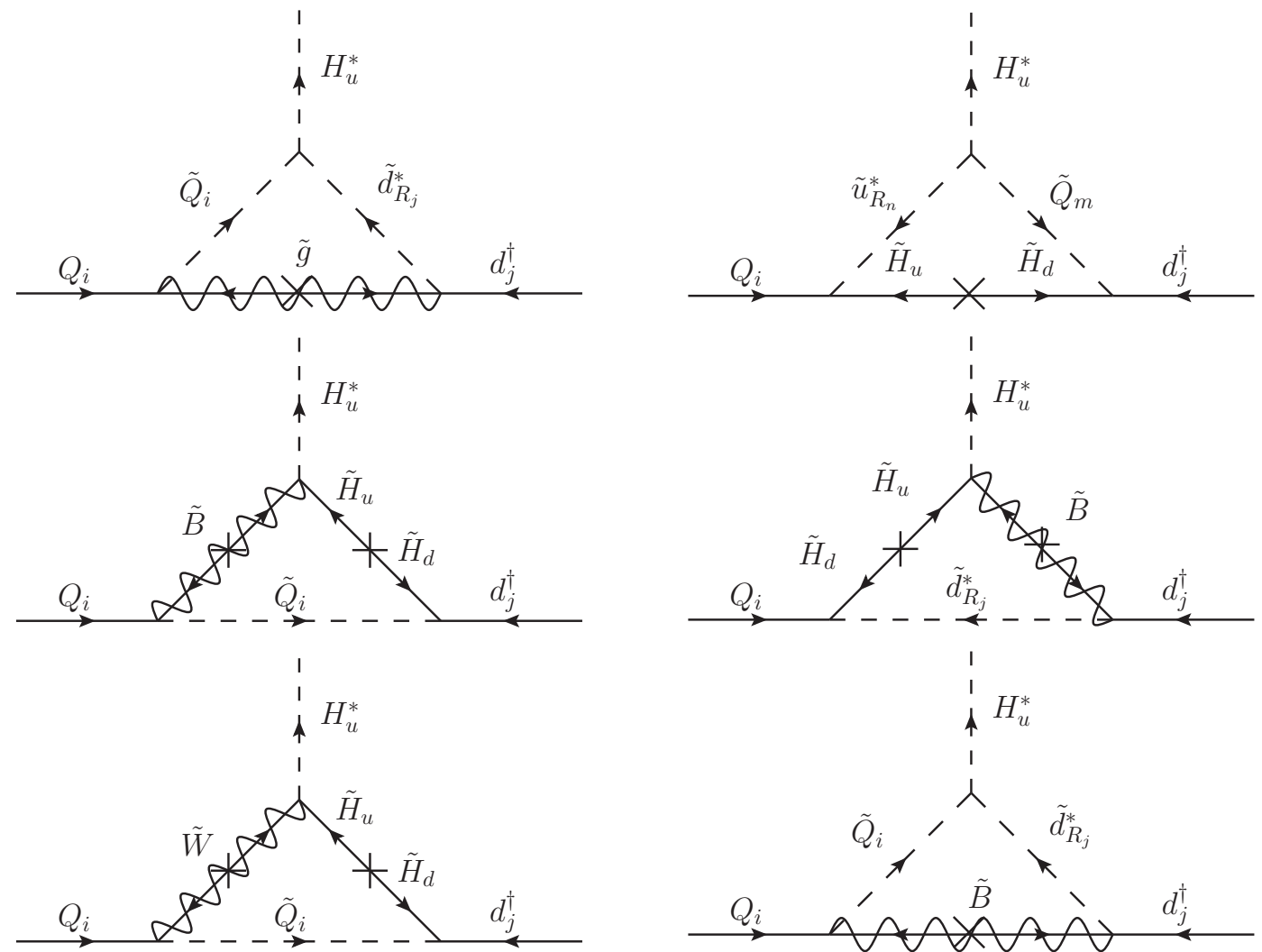

Figure 2. $\tan \beta$-enhanced SUSY threshold corrections to $Y_{d}$.

where $h$ and $H$ are the mass eigenstates doublets of the MSSM Higgs scalar fields and

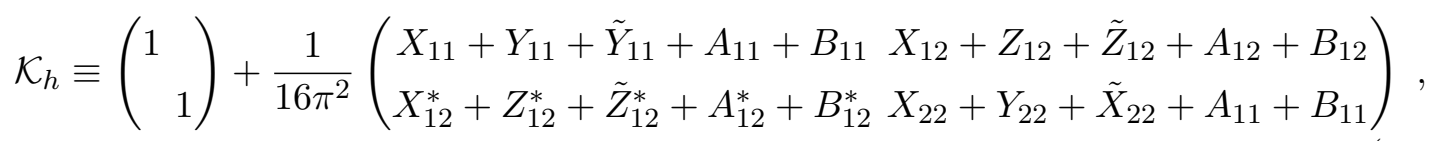

with

$$
\begin{aligned}
& X_{11}=\sum_{i, j}|\mu|^{2}\left|\tilde{Y}_{u_{i j}}\right|^{2} B_{2}\left(m_{\tilde{u}_{i}}^{2}, m_{\tilde{Q}_{j}}^{2}\right), \\
& Y_{11}=\sum_{i, j}\left|\tilde{T}_{d_{i j}}\right|^{2} B_{2}\left(m_{\tilde{d}_{i}}^{2}, m_{\tilde{Q}_{j}}^{2}\right), \\
& \tilde{Y}_{11}=\sum_{i, j}\left|\tilde{T}_{e_{i j}}\right|^{2} B_{2}\left(m_{\tilde{e}_{i}}^{2}, m_{\tilde{L}_{j}}^{2}\right), \\
& X_{12}=\sum_{i, j}-\mu \tilde{T}_{u_{i j}} \tilde{Y}_{u_{i j}}^{*} B_{2}\left(m_{\tilde{u}_{i}}^{2}, m_{\tilde{Q}_{j}}^{2}\right), \\
& \tilde{Z}_{12}=\sum_{i, j}-\mu \tilde{T}_{d_{i j}} \tilde{Y}_{d_{i j}}^{*} B_{2}\left(m_{\tilde{d}_{i}}^{2}, m_{\tilde{Q}_{j}}^{2}\right), \mu \tilde{T}_{e_{i j}} \tilde{Y}_{e_{i j}}^{*} B_{2}\left(m_{\tilde{e}_{i}}^{2}, m_{\tilde{L}_{j}}^{2}\right),
\end{aligned}
$$



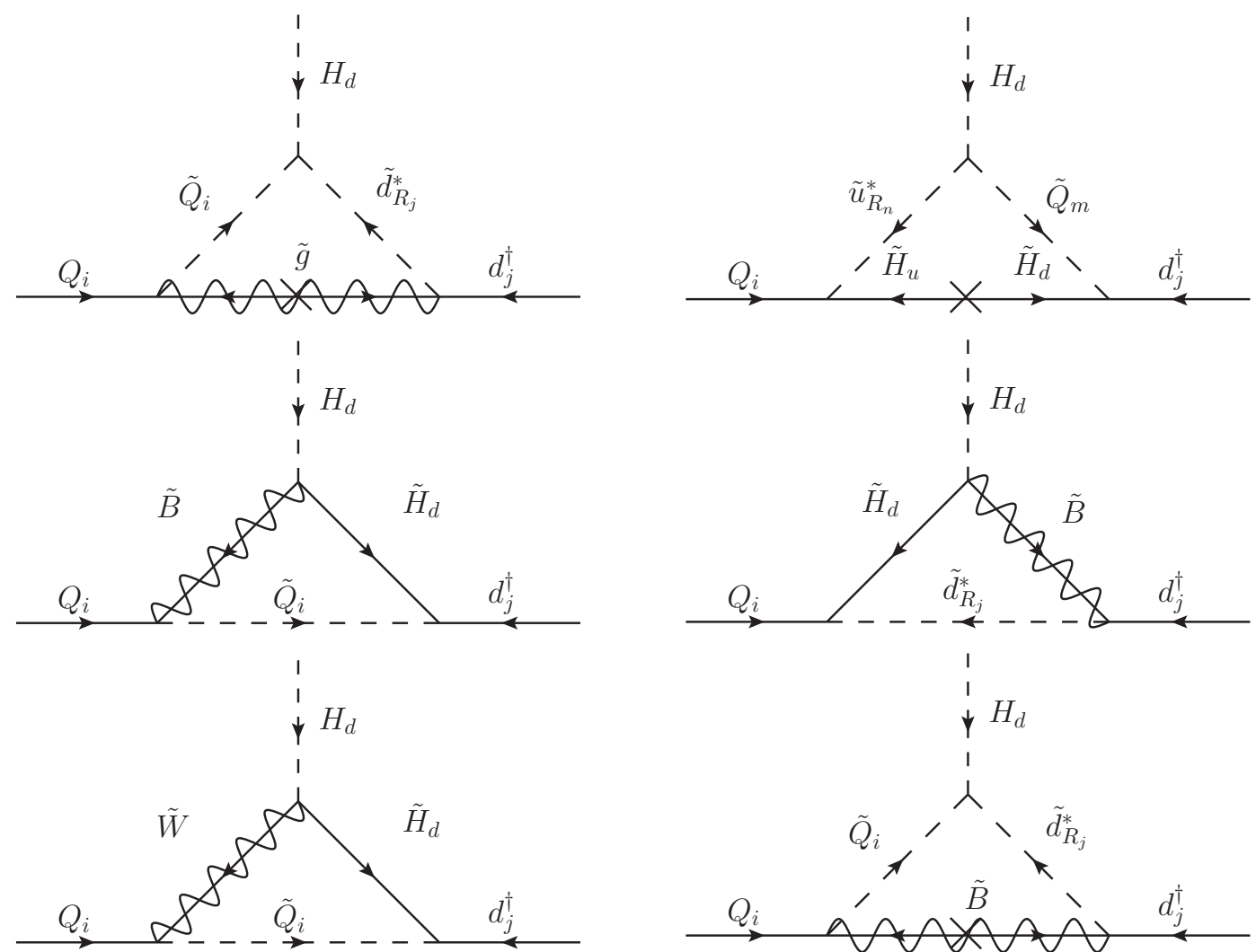

Figure 3. None tan $\beta$-enhanced SUSY threshold corrections to $Y_{d}$.

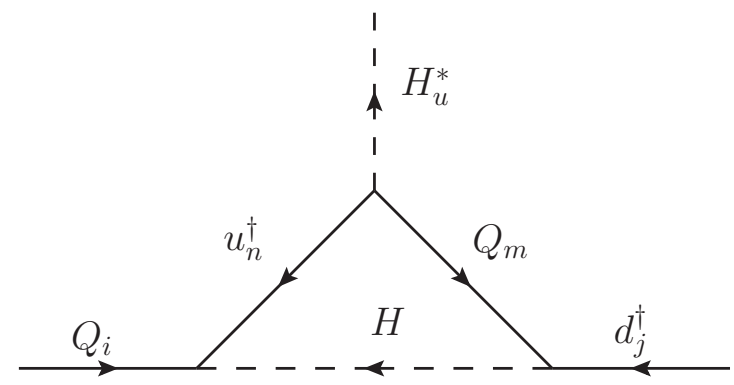

Figure 4. Additional diagram with heavy Higgs doublet exchanged.

$$
\begin{aligned}
X_{22} & =\sum_{i, j}|\mu|^{2}\left|\tilde{Y}_{d_{i j}}\right|^{2} B_{2}\left(m_{\tilde{d}_{i}}^{2}, m_{\tilde{Q}_{j}}^{2}\right), \\
\tilde{X}_{22} & =\sum_{i, j}|\mu|^{2}\left|\tilde{Y}_{e_{i j}}\right|^{2} B_{2}\left(m_{\tilde{e}_{i}}^{2}, m_{\tilde{L}_{j}}^{2}\right), \\
Y_{22} & =\sum_{i, j}\left|\tilde{T}_{u_{i j}}\right|^{2} B_{2}\left(m_{\tilde{u}_{i}}^{2}, m_{\tilde{Q}_{j}}^{2}\right), \\
A_{11} & =\frac{3}{5} g_{1}^{2} \frac{1}{12\left(|\mu|^{2}-\left|M_{1}\right|^{2}\right)^{3}} \tilde{B}_{2}\left(Q^{2},\left|M_{1}\right|^{2},|\mu|^{2}\right),
\end{aligned}
$$




$$
\begin{aligned}
& B_{11}=g_{2}^{2} \frac{1}{4\left(|\mu|^{2}-\left|M_{2}\right|^{2}\right)^{3}} \tilde{B}_{2}\left(Q^{2},\left|M_{2}\right|^{2},|\mu|^{2}\right), \\
& A_{12}=\frac{3}{5} g_{1}^{2} \mu^{*} M_{1}^{*} B_{2}\left(|\mu|^{2},\left|M_{1}\right|^{2}\right) \\
& B_{12}=g_{2}^{2} 3 \mu^{*} M_{2}^{*} B_{2}\left(|\mu|^{2},\left|M_{2}\right|^{2}\right),
\end{aligned}
$$

which correspond to the loops shown in figures 5 and 6 . The loop functions $B_{2}$ and $\tilde{B}_{2}$ are given by

$$
\begin{aligned}
B_{2}(x, y) & \equiv \frac{x^{2}-y^{2}}{2(x-y)^{3}}+\frac{x y}{(x-y)^{3}} \log \left(\frac{x}{y}\right) \\
\tilde{B}_{2}(q, x, y) & \equiv 5\left(x^{3}-y^{3}\right)+27 x y(y-x)+6 y^{2}(y-3 x) \log \left(\frac{y}{x}\right)+6(y-x)^{3} \log \left(\frac{x}{q}\right)
\end{aligned}
$$

The threshold corrections $P_{f}$ due to canonical normalization of the external fermion fields are given by

$$
P_{f} \equiv \sqrt{\left(\mathcal{K}_{f}^{\text {diag }}\right)^{-1}} U_{f} \quad \text { with } \quad \mathcal{K}_{f}^{\text {diag }}=U_{f} \mathcal{K}_{f} U_{f}^{\dagger}
$$

For the threshold corrections of the down-type Yukawa matrix the $\mathcal{K}_{f}$ are given by

$$
\mathcal{K}_{Q_{i j}}=\delta_{i j}+\frac{1}{16 \pi^{2}}\left(\left(\rho_{3}+\rho_{2}+\rho_{1}\right) \delta_{i j}+\rho_{i j}^{d}+\tilde{\rho}_{i j}^{d}+\rho_{i j}^{u}+\tilde{\rho}_{i j}^{u}\right)
$$

with

$$
\begin{aligned}
\rho_{3} & =\frac{8}{3} g_{3}^{2} B_{3}\left(Q^{2},\left|M_{3}\right|^{2}, m_{\tilde{Q}_{j}}^{2}\right) \\
\rho_{2} & =\frac{3}{2} g_{2}^{2} B_{3}\left(Q^{2},\left|M_{2}\right|^{2}, m_{\tilde{Q}_{j}}^{2}\right), \\
\rho_{1} & =\frac{1}{18} \frac{3}{5} g_{1}^{2} B_{3}\left(Q^{2},\left|M_{1}\right|^{2}, m_{\tilde{Q}_{j}}^{2}\right), \\
\rho_{i j}^{d} & =\sum_{k} \tilde{Y}_{d_{j k}}^{\dagger} \tilde{Y}_{d_{k i}} B_{3}\left(Q^{2},|\mu|^{2}, m_{\tilde{d}_{k}}^{2}\right), \\
\tilde{\rho}_{i j}^{d} & =\sum_{k} \tilde{Y}_{d_{j k}}^{\dagger} \tilde{Y}_{d_{k i}} \tilde{B}_{1}\left(\frac{m_{H}^{2}}{Q^{2}}\right) \sin ^{2} \beta, \\
\rho_{i j}^{u} & =\sum_{k} \tilde{Y}_{u_{j k}}^{\dagger} \tilde{Y}_{u_{k i}} B_{3}\left(Q^{2},|\mu|^{2}, m_{\tilde{u}_{k}}^{2}\right), \\
\tilde{\rho}_{i j}^{u} & =\sum_{k} \tilde{Y}_{u_{j k}}^{\dagger} \tilde{Y}_{u_{k i}} \tilde{B}_{1}\left(\frac{m_{H}^{2}}{Q^{2}}\right) \cos ^{2} \beta,
\end{aligned}
$$

corresponding to the loops in figure 7 , and

$$
\mathcal{K}_{d_{i j}}=\delta_{i j}+\frac{1}{16 \pi^{2}}\left(\left(\chi_{3}+\chi_{1}\right) \delta_{i j}+\chi_{i j}^{d}+\tilde{\chi}_{i j}^{d}\right)
$$




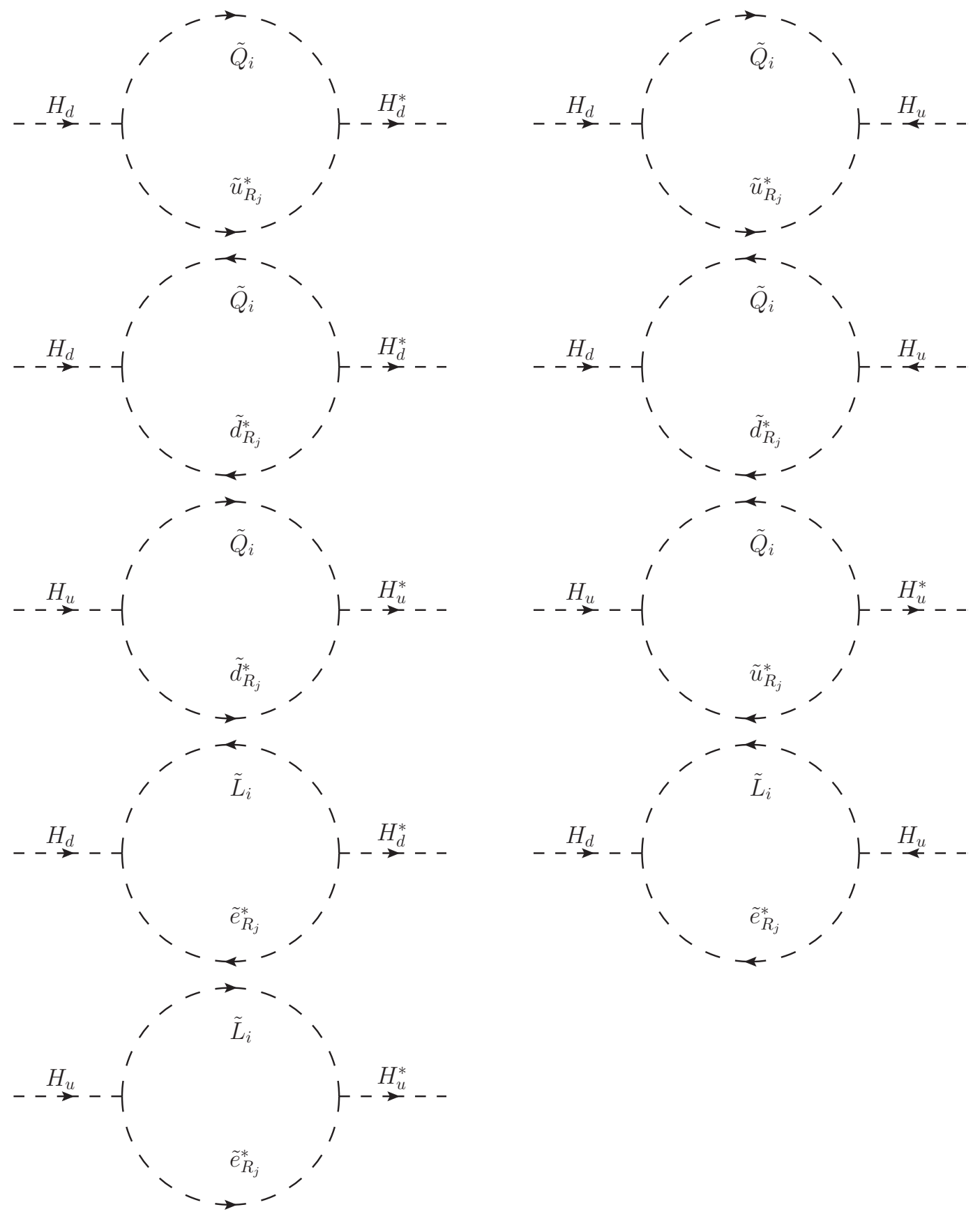

Figure 5. Scalar loops contributing to threshold corrections from canonical normalization of external Higgs fields. 

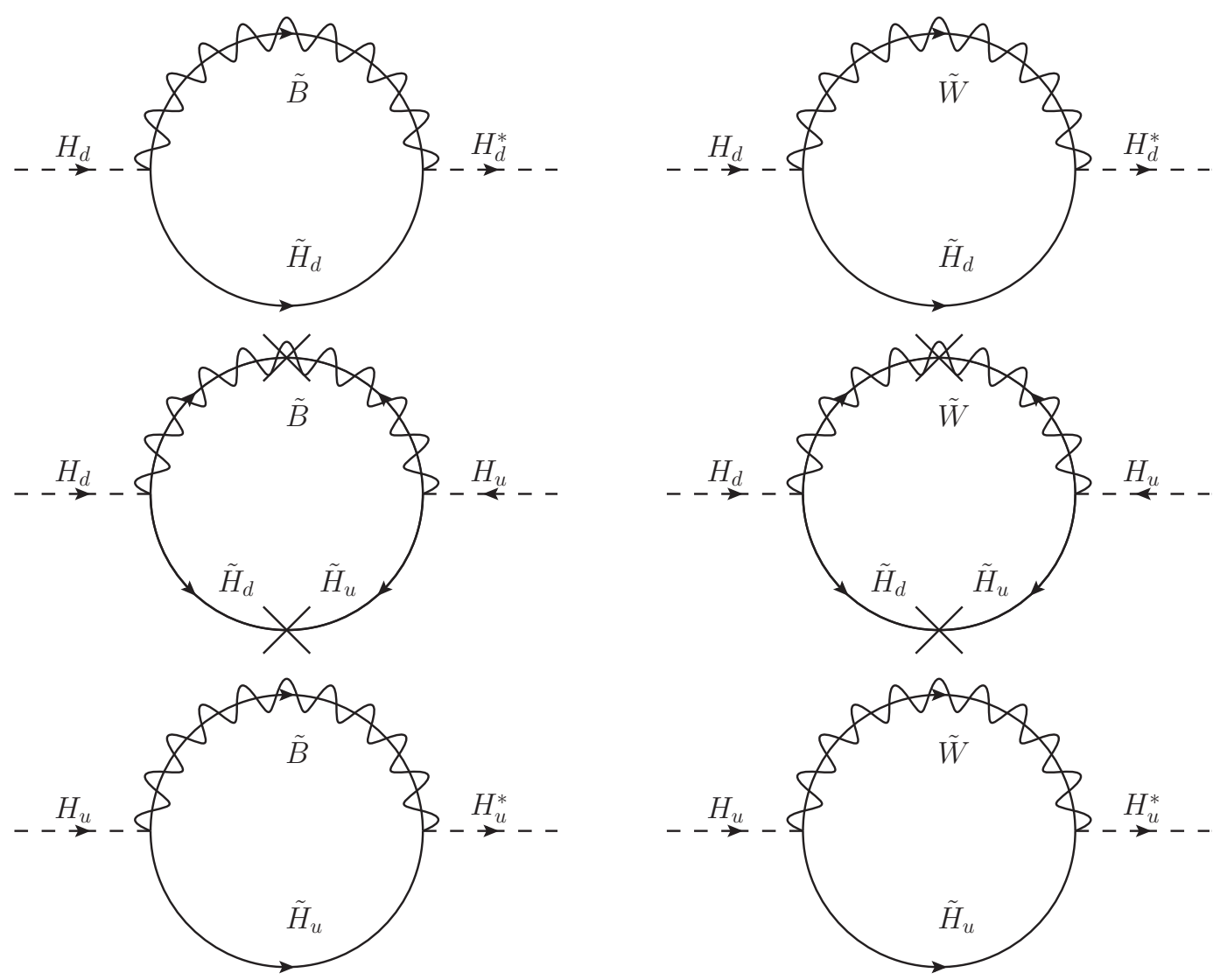

Figure 6. Fermion loops contributing to threshold corrections from canonical normalization of external Higgs fields.

with

$$
\begin{aligned}
\chi_{3} & =\frac{8}{3} g_{3}^{2}\left(Q^{2},\left|M_{3}\right|^{2}, m_{\tilde{d}_{j}}^{2}\right), \\
\chi_{1} & =\frac{2}{9} \frac{3}{5} g_{1}^{2}\left(Q^{2},\left|M_{1}\right|^{2}, m_{\tilde{d}_{j}}^{2}\right), \\
\chi_{i j}^{d} & =\sum_{k} 2 \tilde{Y}_{d_{i k}} \tilde{Y}_{d_{k j}}^{\dagger} B_{3}\left(Q^{2},|\mu|^{2}, m_{\tilde{Q}_{k}}^{2}\right), \\
\tilde{\chi}_{i j}^{d} & =\sum_{k} 2 \tilde{Y}_{d_{i k}} \tilde{Y}_{d_{k j}}^{\dagger} \tilde{B}_{1}\left(\frac{m_{H}^{2}}{Q^{2}}\right) \sin ^{2} \beta .
\end{aligned}
$$

The diagrams for $\mathcal{K}_{d}$ are similar to the ones for $\mathcal{K}_{Q}$, when $Q$ is replaced with $d$, with the exception of the wino loop, which does not exist for external right-handed fermions. The loop-functions $\tilde{B}_{1}$ and $B_{3}$ are given by

$$
\begin{aligned}
\tilde{B}_{1}(q) & \equiv-\frac{1}{4}+\frac{1}{2} \log (q), \\
B_{3}(q, x, y) & \equiv-\frac{1}{4}-\frac{x}{2(x-y)}+\frac{x^{2}}{2(x-y)^{2}} \log \left(\frac{x}{y}\right)+\frac{1}{2} \log \left(\frac{y}{q}\right) .
\end{aligned}
$$




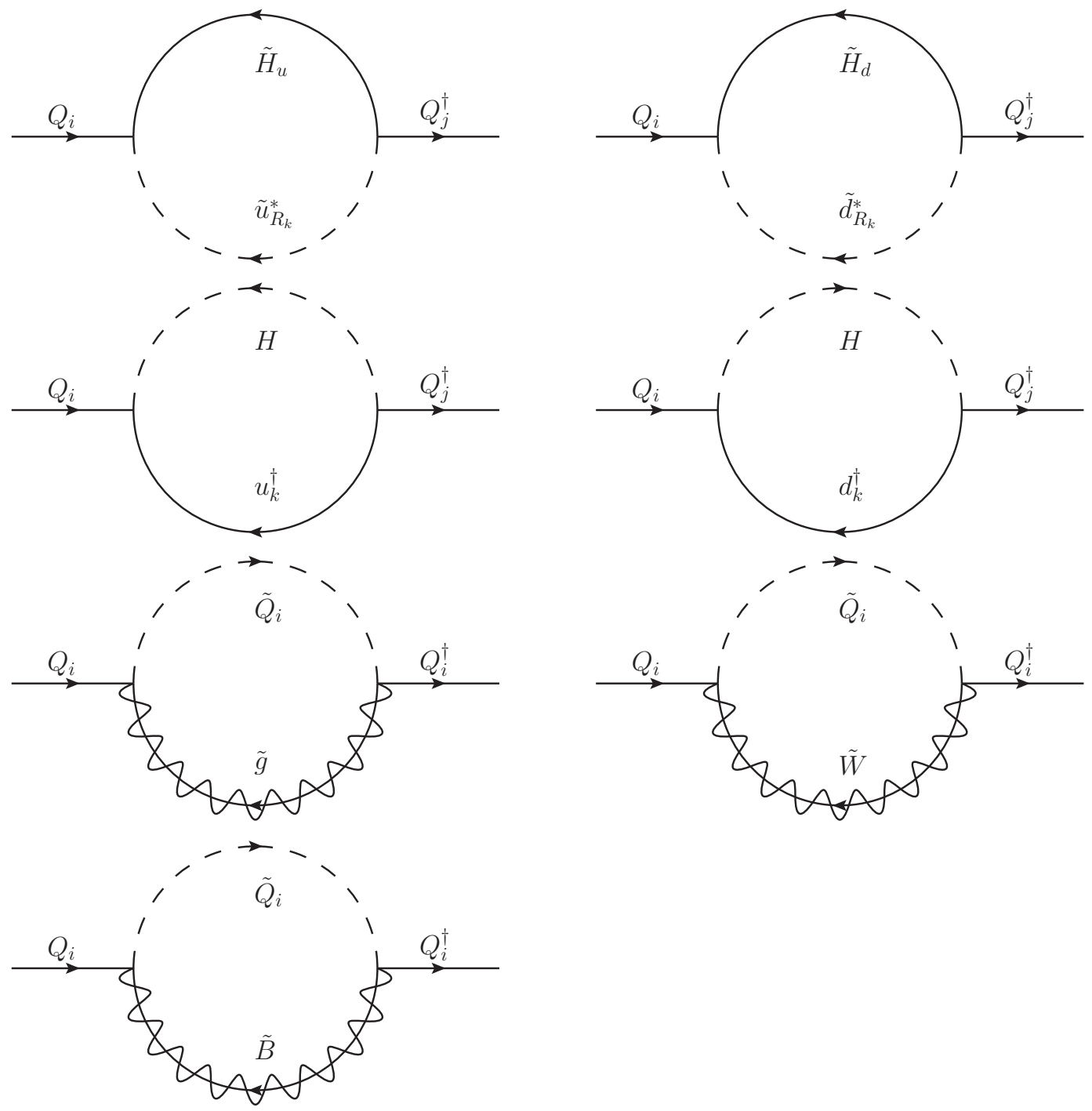

Figure 7. Loops contributing to threshold corrections from canonical normalization of external quark doublets.

For the charged leptons, $Y_{e}^{\mathrm{SM}}$ is given by

$$
\tilde{Y}_{e}^{\mathrm{SM}}=P_{e} \tilde{Y}_{e}^{\prime} P_{L}^{T} P_{h},
$$

where $P_{e}$ and $P_{L}$ are threshold corrections due to canonical normalization of external charged lepton fields and

$$
\begin{aligned}
\tilde{Y}_{e_{i j}}^{\prime}= & \tilde{Y}_{e_{i j}}^{\mathrm{MSSM}} \cos \beta\left(1+\frac{1}{16 \pi^{2}} \tan \beta\left(\tau_{i j}^{W}+\tau_{i j}^{B}\right)+\frac{1}{16 \pi^{2}}\left(\delta_{i j}^{W}+\delta_{i j}^{B}\right)\right) \\
& +\tilde{T}_{e_{i j}}^{\mathrm{MSSM}} \cos \beta \frac{1}{16 \pi^{2}} \xi_{i j}^{B}
\end{aligned}
$$


with the $\tan \beta$-enhanced contributions

$$
\begin{aligned}
\tau_{i j}^{W} & =\frac{3}{2} g_{2}^{2} \frac{M_{2}^{*}}{\mu} H_{2}\left(\frac{\left|M_{2}\right|^{2}}{|\mu|^{2}}, \frac{m_{\tilde{L}_{j}}^{2}}{|\mu|^{2}}\right), \\
\tau_{i j}^{B} & =\frac{3}{5} g_{1}^{2}\left(-\frac{\mu^{*}}{M_{1}} H_{2}\left(\frac{m_{\tilde{e}_{i}}^{2}}{\left|M_{1}\right|^{2}}, \frac{m_{\tilde{L}_{j}}^{2}}{\left|M_{1}\right|^{2}}\right)+\frac{M_{1}^{*}}{\mu} H_{2}\left(\frac{m_{\tilde{e}_{i}}^{2}}{|\mu|^{2}}, \frac{\left|M_{1}\right|^{2}}{|\mu|^{2}}\right)-\frac{1}{2} \frac{M_{1}^{*}}{\mu} H_{2}\left(\frac{\left|M_{1}\right|^{2}}{|\mu|^{2}}, \frac{m_{\tilde{L}_{j}}^{2}}{|\mu|^{2}}\right)\right),
\end{aligned}
$$

and

$$
\begin{aligned}
\delta_{i j}^{W} & =12 g_{2}^{2} C_{00}\left(\frac{|\mu|^{2}}{Q^{2}}, \frac{\left|M_{2}\right|^{2}}{|\mu|^{2}}, \frac{m_{\tilde{L}_{j}}^{2}}{|\mu|^{2}}\right), \\
\delta_{i j}^{B} & =\frac{3}{5} g_{1}^{2}\left(-2 C_{00}\left(\frac{|\mu|^{2}}{Q^{2}}, \frac{\left|M_{1}\right|^{2}}{|\mu|^{2}}, \frac{m_{\tilde{L}_{j}}^{2}}{|\mu|^{2}}\right)+4 C_{00}\left(\frac{|\mu|^{2}}{Q^{2}}, \frac{\left|M_{1}\right|^{2}}{|\mu|^{2}}, \frac{m_{\tilde{e}_{i}}^{2}}{|\mu|^{2}}\right)\right), \\
\xi_{i j}^{B} & =\frac{3}{5} g_{1}^{2} \frac{1}{M_{1}} H_{2}\left(\frac{m_{\tilde{e}_{i}}^{2}}{\left|M_{1}\right|^{2}}, \frac{m_{\tilde{L}_{j}}^{2}}{\left|M_{1}\right|^{2}}\right) .
\end{aligned}
$$

The diagrams for the $Y_{e}$ SUSY threshold corrections are analogous to the ones in figures 2 and 3 , with the exception that the loop diagrams shown in the top rows do not exist. The diagram of figure 4 also doesn't have an analogue for $Y_{e} . P_{e}$ and $P_{L}$ are calculated from (3.19) with

$$
\mathcal{K}_{L}=\delta_{i j}+\frac{1}{16 \pi^{2}}\left(\left(\tilde{\rho}_{2}+\tilde{\rho}_{1}\right) \delta_{i j}+\rho_{i j}^{e}+\tilde{\rho}_{i j}^{e}\right),
$$

where

$$
\begin{aligned}
\tilde{\rho}_{2} & =\frac{3}{2} g_{2}^{2} B_{3}\left(Q^{2},\left|M_{2}\right|^{2}, m_{\tilde{L}_{j}}^{2}\right), \\
\tilde{\rho}_{1} & =\frac{1}{2} \frac{3}{5} g_{1}^{2} B_{3}\left(Q^{2},\left|M_{1}\right|^{2}, m_{\tilde{L}_{j}}^{2}\right), \\
\rho_{i j}^{e} & =\sum_{k} \tilde{Y}_{e_{j k}}^{\dagger} \tilde{Y}_{e_{k i}} B_{3}\left(Q^{2},|\mu|^{2}, m_{\tilde{e}_{k}}^{2}\right), \\
\tilde{\rho}_{i j}^{e} & =\sum_{k} \tilde{Y}_{e_{j k}}^{\dagger} \tilde{Y}_{e_{k i}} \tilde{B}_{1}\left(\frac{m_{H}^{2}}{Q^{2}}\right) \sin ^{2} \beta,
\end{aligned}
$$

and

$$
\mathcal{K}_{e}=\delta_{i j}+\frac{1}{16 \pi^{2}}\left(\tilde{\chi}_{1} \delta_{i j}+\chi_{i j}^{e}+\tilde{\chi}_{i j}^{e}\right)
$$

with

$$
\begin{aligned}
\tilde{\chi}_{1} & =2 \frac{3}{5} g_{1}^{2} B_{3}\left(Q^{2},\left|M_{1}\right|^{2}, m_{\tilde{e}_{i}}^{2}\right), \\
\chi_{i j}^{e} & =\sum_{k} 2 \tilde{Y}_{e_{i k}} \tilde{Y}_{e_{k j}}^{\dagger} B_{3}\left(Q^{2},|\mu|^{2}, m_{\tilde{L}_{k}}^{2}\right), \\
\tilde{\chi}_{i j}^{e} & =\sum_{k} \tilde{2} Y_{e_{i k}} \tilde{Y}_{e_{k j}}^{\dagger} \tilde{B}_{1}\left(\frac{m_{H}^{2}}{Q^{2}}\right) \sin ^{2} \beta .
\end{aligned}
$$


Again, the loop diagrams for $\mathcal{K}_{L}$ and $\mathcal{K}_{e}$ can be easily obtained from the diagrams for $\mathcal{K}_{Q}$ by suitable exchange of labels and indices and dropping non-existent gaugino loops.

Turning to $Y_{u}$, the types of diagrams which were $\tan \beta$-enhanced for $Y_{d}$ and $Y_{e}$ are now $\tan \beta$-suppressed. However, there also exist SUSY threshold corrections which are independent of $\tan \beta$ and enhanced by large trilinear couplings. These SUSY threshold corrections to $Y_{u}$ can have important effects. For example the SUSY threshold corrections to the top Yukawa coupling $y_{t}$ can be of significance in analyses of the Higgs mass and vacuum stability. The expression for the $Y_{u}$ SUSY threshold corrections can be readily obtained from the SUSY threshold corrections to $Y_{d}(3.4)-(3.7)$ and (3.19)-(3.23) by the replacement ${ }^{5}$

$$
\begin{aligned}
d & \rightarrow u, \\
\cos \beta & \rightarrow \sin \beta,
\end{aligned}
$$

with the exception of the bino-loops, whose contribution become

$$
\begin{aligned}
\eta_{i j}^{B} & =\frac{3}{5} g_{1}^{2}\left(-\frac{2}{9} \frac{\mu^{*}}{M_{1}} H_{2}\left(\frac{m_{\tilde{d}_{i}}^{2}}{\left|M_{1}\right|^{2}}, \frac{m_{\tilde{Q}_{j}}^{2}}{\left|M_{1}\right|^{2}}\right)+\frac{2}{3} \frac{M_{1}^{*}}{\mu} H_{2}\left(\frac{m_{\tilde{\tilde{u}}_{i}}^{2}}{|\mu|^{2}}, \frac{\left|M_{1}\right|^{2}}{|\mu|^{2}}\right)-\frac{1}{6} \frac{M_{1}^{*}}{\mu} H_{2}\left(\frac{\left|M_{1}\right|^{2}}{|\mu|^{2}}, \frac{m_{\tilde{Q}_{j}}^{2}}{|\mu|^{2}}\right)\right), \\
\epsilon_{i j}^{B} & =\frac{3}{5} g_{1}^{2}\left(\frac{8}{3} C_{00}\left(\frac{|\mu|^{2}}{Q^{2}}, \frac{\left|M_{1}\right|^{2}}{|\mu|^{2}}, \frac{m_{\tilde{u}_{i}}^{2}}{|\mu|^{2}}\right)-\frac{2}{3} C_{00}\left(\frac{|\mu|^{2}}{Q^{2}}, \frac{\left|M_{1}\right|^{2}}{|\mu|^{2}}, \frac{m_{\tilde{Q}_{j}}^{2}}{|\mu|^{2}}\right)\right), \\
\zeta_{i j}^{B} & =\frac{3}{5} g_{1}^{2} \frac{2}{9} \frac{1}{M_{1}} H_{2}\left(\frac{m_{\tilde{u}_{i}}^{2}}{\left|M_{1}\right|^{2}}, \frac{m_{\tilde{Q}_{j}}^{2}}{\left|M_{1}\right|^{2}}\right), \\
\chi_{1} & =\frac{8}{9} \frac{3}{5} g_{1}^{2}\left(Q^{2},\left|M_{1}\right|^{2}, m_{\tilde{u}_{i}}^{2}\right),
\end{aligned}
$$

due to the different $\mathrm{U}(1)$ hypercharges of the (s)particles in the loop. The loop diagrams are identical to the ones of figures 2,3 , and 4 with $u$ and $d$ exchanged.

After the SUSY threshold corrections are incorporated in the $\overline{\mathrm{DR}}$ scheme, REAP converts the Yukawa and gauge couplings to the $\overline{\mathrm{MS}}$ scheme following [58].

Finally SusyTC calculates the value of $|\mu|$ and $m_{3}$ from $m_{H_{u}}^{2}, m_{H_{d}}^{2}, \tan \beta$ and $M_{Z}$ by requiring the existence of spontaneously broken EW vacuum, which is equivalent to vanishing one-loop corrected tad-pole equations of $H_{u}$ and $H_{d}$

$$
\mu=\mathrm{e}^{i \phi_{\mu}} \sqrt{\frac{1}{2}\left(\tan (2 \beta)\left(\bar{m}_{H_{u}}^{2} \tan \beta-\bar{m}_{H_{d}}^{2} \cot \beta\right)-M_{Z}^{2}-\operatorname{Re}\left(\Pi_{Z Z}^{T}\left(M_{Z}^{2}\right)\right)\right)}
$$

with $\bar{m}_{H_{u}}^{2} \equiv m_{H_{u}}^{2}-t_{u}$ and $\bar{m}_{H_{d}}^{2} \equiv m_{H_{d}}^{2}-t_{d}$. In the real (CP conserving) MSSM the phase $\phi_{\mu}$ is restricted to 0 and $\pi$. The expressions for the one-loop tadpoles $t_{u}, t_{d}$ and the transverse Z-boson self energy $\Pi_{Z Z}^{T}$ are based on [59], but extended to include inter-generational mixing, and are presented in appendix B. Because $\mu$ enters the one-loop formulas for the threshold corrections, treating $t_{u}, t_{d}$ and $\Pi_{Z Z}^{T}$ as functions of tree-level parameters is sufficiently accurate. The one-loop expression of the soft-breaking mass $m_{3}$ is calculated as

$$
m_{3}=\sqrt{\frac{1}{2}\left(\tan (2 \beta)\left(\bar{m}_{H_{u}}^{2}-\bar{m}_{H_{d}}^{2}\right)-\left(M_{Z}^{2}+\operatorname{Re}\left(\Pi_{Z Z}^{T}\left(M_{Z}^{2}\right)\right)\right) \sin (2 \beta)\right)} .
$$

\footnotetext{
${ }^{5}$ Note that $(3.12)-(3.15)$ stay invariant.
} 
If desired, SusyTC allows to outsource a two-loop Higgs mass calculation to external software, e.g. FeynHiggs [60-67], by calculating the pole mass $m_{H^{+}}\left(m_{A}\right)$ as input for the complex (real) MSSM

$$
\begin{aligned}
m_{H^{+}}^{2}=\frac{1}{\cos (2 \beta)} & \left(\bar{m}_{H_{u}}^{2}-\bar{m}_{H_{d}}^{2}-M_{Z}^{2}-\operatorname{Re}\left(\Pi_{Z Z}^{T}\left(M_{Z}^{2}\right)\right)+\hat{M}_{W}^{2}\right. \\
& \left.-\operatorname{Re}\left(\Pi_{H^{+} H^{-}}\left(m_{H^{+}}\right)\right)+t_{d} \sin ^{2} \beta+t_{u} \cos ^{2} \beta\right), \\
m_{A}^{2}=\frac{1}{\cos (2 \beta)} & \left(\bar{m}_{H_{u}}^{2}-\bar{m}_{H_{d}}^{2}-M_{Z}^{2}-\operatorname{Re}\left(\Pi_{Z Z}^{T}\left(M_{Z}^{2}\right)\right)\right. \\
& \left.\times \operatorname{Re}\left(\Pi_{A A}\left(m_{A}\right)\right)+t_{d} \sin ^{2} \beta+t_{u} \cos ^{2} \beta\right),
\end{aligned}
$$

where $\hat{M}_{W}$ is the $\overline{\mathrm{DR}} \mathrm{W}$-boson mass given as

$$
\hat{M}_{W}^{2}(Q)=M_{W}^{2}+\operatorname{Re}\left(\Pi_{W W}^{T}\left(M_{W}^{2}\right)\right)=g_{2} \frac{\hat{v}(Q)}{2},
$$

with $M_{Z}$ and $M_{W}$ pole masses and the $\overline{\mathrm{DR}}$ vacuum expectation value $\hat{v}(Q)$ given by

$$
\hat{v}^{2}(Q)=4 \frac{M_{Z}^{2}+R e\left(\Pi_{Z Z}^{T}\left(M_{Z}^{2}\right)\right)}{\frac{3}{5} g_{1}^{2}(Q)+g_{2}^{2}(Q)} .
$$

As in the previous formulas, the self energies $\Pi_{H^{+} H^{-}}$and $\Pi_{A A}$ are based on [59], but extended to include inter-generational mixing, and are understood as functions of treelevel parameters. They are given in appendix B.

\section{The REAP extension SusytC}

In this section we provide a "Getting Started" calculation for SusyTC. A full documentation of all features is included in appendix C. Since SusyTC is an extension to REAP, an up-todate version of REAP-MPT [1] (available at http://reapmpt.hepforge.org) needs to be installed on your system. SusyTC consists out of the REAP model file RGEMSSMsof tbroken.m, which is based on the model file RGEMSSM.m of REAP 1.11.2 and additionally contains, among other things, the RGEs of the MSSM soft-breaking parameters and the matching to the SM, and the file SusyTC.m, which includes the formulas for the sparticle spectrum and SUSY threshold correction calculations. Both files can be downloaded from http://particlesandcosmology. unibas.ch/pages/SusyTC.htm and have to be copied into the REAP directory.

To begin a calculation with SusyTC, one first needs to import RGEMSSMsoftbroken.m:

$$
\text { Needs["REAP'RGEMSSMsof tbroken'"]; }
$$

The model MSSMsoftbroken is then defined by RGEAdd, including additional options such as RGEtan $\beta$ :

RGEAdd["MSSMsoftbroken",RGEtan $\beta \rightarrow 30] ;$ 
In MSSMsof tbroken all REAP options of the model MSSM are available. The options additionally available in SusyTC are given in appendix C. The input is given by RGESetInitial. Let us illustrate some features of SusyTC: to test for example the GUT scale prediction for the Yukawa coupling ratio $\frac{y_{\mu}}{y_{s}}=6$, considering a given example parameter point in the Constrained MSSM, one can type:

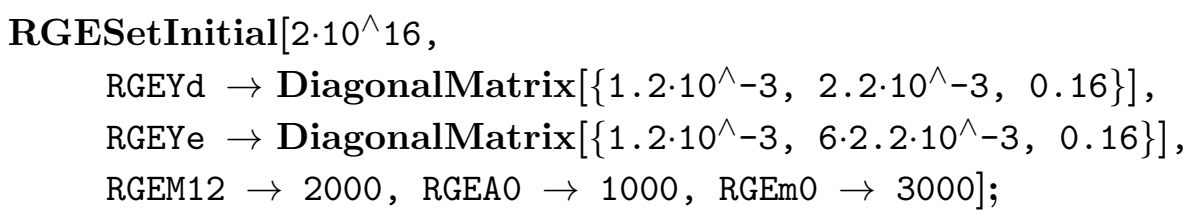

Of course, any general matrices can be used as input for the Yukawa, trilinear, and softbreaking matrices, as given by the specific SUSY flavour GUT model under consideration. Also, non-universal gaugino masses can be specified. As in REAP, dimensional quantities are given in units of GeV. The RGEs are then solved from the GUT scale to the Z-boson mass scale by

$$
\text { RGESsolve[91, 2.10^16]; }
$$

The ratio of the $\mu$ and strange quark Yukawa couplings at the Z-boson mass scale can now be obtained with RGEGetSolution, CKMParameters, and MNSParameters:

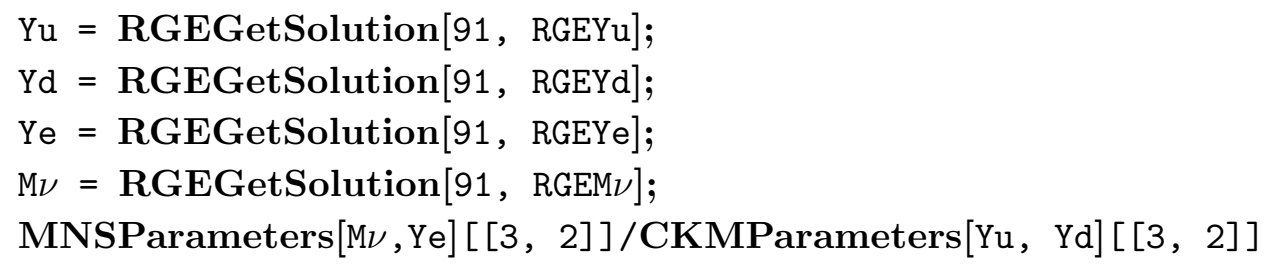

Repeating this calculation with all SU(5) CG factors listed in table 2 of [12], one obtains the results shown in figure 8 .

As described in appendix C, SusyTC can also read and write "Les Houches" files $[55,58]$ as input and output.

\section{The sparticle spectrum predicted from CG factors}

In this section we apply SusyTC to investigate the constraints on the sparticle spectrum which arise from a set of GUT scale predictions for the quark-lepton Yukawa coupling ratios $\frac{y_{e}}{y_{d}}, \frac{y_{\mu}}{y_{s}}$, and $\frac{y_{\tau}}{y_{b}}$. As GUT scale boundary conditions for the SUSY-breaking terms we take the Constrained MSSM. The experimental constraints are given by the Higgs boson mass $m_{h^{0}}=$ $125.09 \pm 0.21 \pm 0.11 \mathrm{GeV}[68]$ as well as the charged fermion masses (and the quark mixing matrix). We use the experimental constraints for the running $\overline{\mathrm{MS}}$ Yukawa couplings at the Z-boson mass scale calculated in [36], where we set the uncertainty of the charged lepton Yukawa couplings to one percent to account for the estimated theoretical uncertainty (which here exceeds the experimental uncertainty). When applying the measured Higgs mass as constraint, we use a $1 \sigma$ interval of $\pm 3 \mathrm{GeV}$, including the estimated theoretical uncertainty. 


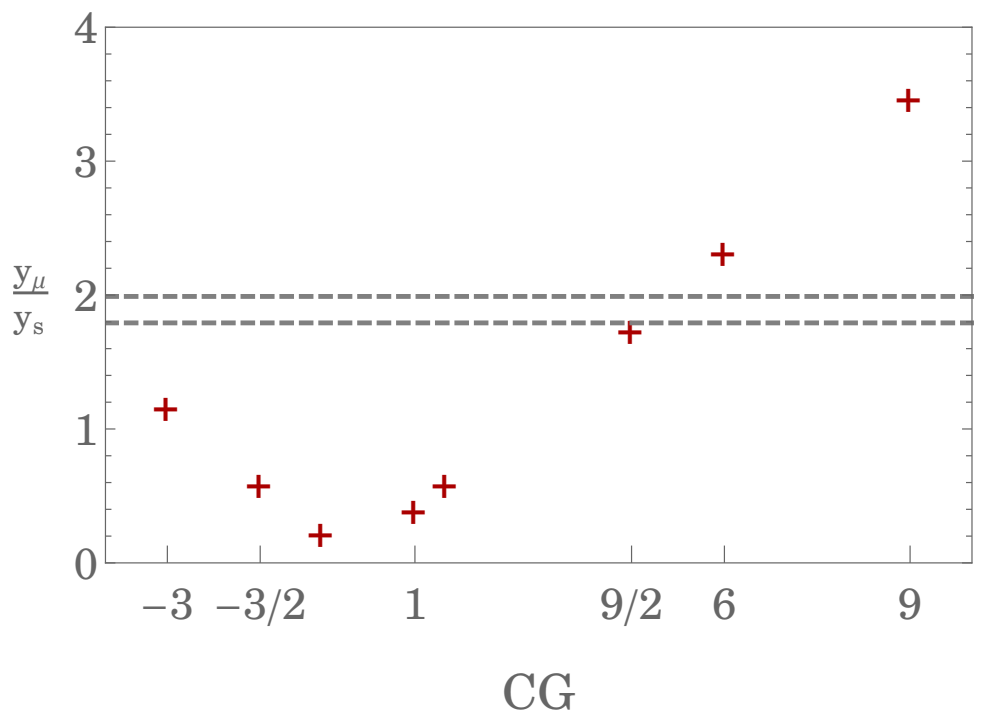

Figure 8. Example results for $\frac{y_{\mu}}{y_{s}}$ at the electroweak scale, considering the SU(5) GUT-scale CG factors from table 2 of [12], i.e. the GUT predictions $\frac{y_{\mu}}{y_{s}}=\mathrm{CG}$, for a given example Constrained MSSM parameter point with $\tan \beta=30, m_{1 / 2}=2000 \mathrm{GeV}, A_{0}=1000 \mathrm{GeV}$, and $m_{0}=3000 \mathrm{GeV}$. The area between the dashed gray lines corresponds to the experimental $1 \sigma$ range [36].

For our study, we consider GUT scale Yukawa coupling matrices which feature the GUT-scale Yukawa relations $\frac{y_{e}}{y_{d}}=-\frac{1}{2}, \frac{y_{\mu}}{y_{s}}=6$, and $\frac{y_{\tau}}{y_{b}}=-\frac{3}{2}$ (cf. [12]):

$$
\begin{aligned}
& Y_{d}=\left(\begin{array}{ccc}
y_{d} & 0 & 0 \\
0 & y_{s} & 0 \\
0 & 0 & y_{b}
\end{array}\right), \quad Y_{e}=\left(\begin{array}{ccc}
-\frac{1}{2} y_{d} & 0 & 0 \\
0 & 6 y_{s} & 0 \\
0 & 0 & -\frac{3}{2} y_{b}
\end{array}\right), \\
& Y_{u}=\left(\begin{array}{ccc}
y_{u} & 0 & 0 \\
0 & y_{c} & 0 \\
0 & 0 & y_{t}
\end{array}\right) U_{\mathrm{CKM}}\left(\theta_{12}, \theta_{13}, \theta_{23}, \delta\right),
\end{aligned}
$$

These GUT relations can emerge as direct result of CG factors in SU(5) GUTs or as approximate relation after diagonalization of the GUT-scale Yukawa matrices $Y_{d}$ and $Y_{e}$ (cf. $[34,35,43,44]$ ). For the soft-breaking parameters we restrict our analysis to the Constrained MSSM parameters $m_{0}, m_{1 / 2}, A_{0}$ and $\tan \beta$, with $\mu$ determined from requiring the breaking of electroweak symmetry as in (3.36) and set $\operatorname{sgn}(\mu)=+1$. We note that in specific models for the GUT Higgs potential, for instance in [43], $\mu$ can be realized as an effective parameter of the superpotential with a fixed phase, including the case that $\mu$ is real.

We note that we have also added a neutrino sector, i.e. a neutrino Yukawa matrix $Y_{\nu}$ and and a mass matrix $M_{n}$ of the right-handed neutrinos, but we have set the entries of $Y_{\nu}$ to very small values below $\mathcal{O}\left(10^{-3}\right)$, such that their effects on the RG evolution can be safely neglected, and the masses of the right-handed neutrinos to values many orders of magnitude higher than the expected SUSY scale. With these parameters, the neutrino 
sector is decoupled from the main analysis. Such small values of the neutrino Yukawa couplings are e.g. expected in the models [34, 35, 44], where they arise as effective operators.

Using one-loop RGEs, REAP 1.11.3 and SusyTC 1.1 we determine the soft-breaking parameters and $\mu$ at the SUSY scale, as well as the pole mass $m_{H^{+}}$. This output is then passed to FeynHiggs 2.11.3 [60-67] in order to calculate the two-loop corrected pole masses of the Higgs bosons in the complex MSSM. The MSSM is automatically matched to the SM and we compare the results for the Yukawa couplings at the Z-boson mass scale with the experimental values reported in [36].

When fitting the GUT-scale parameters to the experimental data, we found that our results for the up-type quark Yukawa couplings and CKM angles and CP-phase could be fitted to agree with observations to at least $10^{-3}$ relative precision, by adjusting the parameters of $Y_{u}$. The remaining six parameters are used to fit the Yukawa couplings of down-type quarks and charged leptons, as well as the mass of the SM-like Higgs boson.

We find a benchmark point with a $\chi^{2}=0.9$ :

\begin{tabular}{|cccc|}
\hline \multicolumn{4}{|c|}{ input GUT scale parameters } \\
\hline$y_{d}$ & $y_{s}$ & $y_{b}$ \\
$8.92 \cdot 10^{-5}$ & $1.57 \cdot 10^{-3}$ & 0.109 \\
$m_{0}$ & $A_{0}$ & $m_{1 / 2}$ & $\tan \beta$ \\
$1629.48 \mathrm{GeV}$ & $-3152.70 \mathrm{GeV}$ & $1840.48 \mathrm{GeV}$ & 21.27 \\
\hline \multicolumn{4}{c}{} \\
\hline \multicolumn{4}{c}{ low energy results } \\
$2.79 \cdot 10^{-6}$ & $5.90 \cdot 10^{-4}$ & $1.00 \cdot 10^{-2}$ & \\
$y_{d}$ & $y_{s}$ & $y_{b}$ & $m_{h^{0}}$ \\
$1.75 \cdot 10^{-5}$ & $3.07 \cdot 10^{-4}$ & $1.64 \cdot 10^{-2}$ & $123.6 \mathrm{GeV}$ \\
\hline
\end{tabular}

Looking at our results for the low-energy Yukawa coupling ratios, $\frac{y_{e}}{y_{d}}=0.16, \frac{y_{\mu}}{y_{s}}=1.92$, and $\frac{y_{\tau}}{y_{b}}=0.61$, the importance of SUSY threshold corrections in evaluating the GUT-scale Yukawa ratios becomes evident. This can also be seen in figure 9. Additionally, as shown in figure 10, SUSY threshold corrections also affect the CKM mixing angles.

The SUSY spectrum obtained by SusyTC is shown in figure 11. The lightest supersymmetric particle (LSP) is a bino-like neutralino of about $827 \mathrm{GeV}$. The SUSY scale is obtained as $Q=3014 \mathrm{GeV}$. The $\mu$ parameter obtained from requiring spontaneous electroweak symmetry breaking is given by $\mu=2634 \mathrm{GeV}$. Note that the only experimental constraints we used were the results for quark and charged lepton masses as well as $m_{h^{0}}$. In particular, no bounds on the sparticle masses were applied as well as no restrictions from the neutralino relic density (which would require further assumptions on the cosmological evolution) ${ }^{6}$

\footnotetext{
${ }^{6}$ For example, the neutralino relic density may be diluted if additional entropy gets produced at late times. Therefore we do not use the neutralino relic density as a constraint here.
} 
Due to the large (absolute) values of the trilinear couplings, we find using the constraints from [69], that the vacuum of our benchmark point is meta-stable. The scalar potential possesses charge and colour breaking (CCB) vacua, as well as one "unbounded from below" (UFB) field direction in parameter space. However, estimating the stability of the vacuum via the Euclidean action of the "bounce" solution [70, 71] (following [72]) shows that the lifetime of the vacuum is many orders larger than the age of the universe.

Confidence intervals for the sparticle masses are obtained as Bayesian "highest posterior density" (HPD) intervals ${ }^{7}$ from a Markov Chain Monte Carlo sample of 1.2 million points, using a Metropolis algorithm. We note that we did not compute the lifetime of the vacuum for each point in the MCMC analysis, which would take far too much computation time. This means that the obtained confidence intervals should be regarded as conservative, in the sense that including the lifetime constraints the upper bounds on the masses could become smaller. For some example points within the $1 \sigma$ HPD regions we have checked that the lifetime constraints are satisfied. We applied the following priors to the MCMC analysis: $m_{0} \in[0,4] \mathrm{TeV}, A_{0} \in[-10,0] \mathrm{TeV}, m_{1 / 2} \in[0,6] \mathrm{TeV}$ and $\tan \beta \in[2,40]$. As shown in figure 12 our results for the $1 \sigma$ HPD intervals for the Constrained MSSM softbreaking parameters are well within these intervals. The $1 \sigma$ HPD results of the sparticle masses are shown in figure 13. Furthermore we find that for about $70 \%$ of the data points of the MCMC analysis, the lightest MSSM sparticle is a neutralino, while for the others it is the lightest charged slepton. ${ }^{8}$ The HPD interval for the SUSY scale is obtained as $Q_{\mathrm{HPD}}=[2048,5108] \mathrm{GeV}$.

\subsection{Comments and discussion}

We would like to emphasize that the results described above have been obtained under specific assumptions for the input parameters at the GUT scale. In the following, we discuss these assumptions, how they may be obtained and/or generalized in fully worked out models, and also some limitations and uncertainties of our analysis.

To start with, we have chosen the specific GUT scale predictions $\frac{y_{\tau}}{y_{b}}=-\frac{3}{2}, \frac{y_{\mu}}{y_{s}}=6$ and $\frac{y_{e}}{y_{d}}=-\frac{1}{2}$. This is indeed only one of the possible predictions that can arise from GUTs. Other possibilities can be found, e.g., in [11-13]. We have chosen the above set of GUT predictions since they are among the ones recently used successfully in GUT model building (see e.g. [34, 35, 43, 44]). In the future, it will of course be interesting to also test other combinations of promising Clebsch factors, and compare the predictions/constraints on the SUSY spectra. Of course, one can also construct GUT models which do not predict the quark-lepton Yukawa ratios. For such GUT models the constraints discussed here would not apply.

Furthermore, for our study we have assumed CMSSM boundary conditions for the soft breaking parameters, which is quite a strong assumption that will probably often be relaxed in realistic models. On the other hand, universal boundary conditions may also be a result of a specific SUSY breaking mechanism. Apart from the SUSY breaking

\footnotetext{
${ }^{7} \mathrm{An} 1 \sigma$ HPD interval is the interval $\left[\theta_{L}, \theta_{H}\right]$ such that $\int_{\theta_{L}}^{\theta_{H}} p(\theta) d \theta=0.6826 \ldots$ and the posterior probability density $p(\theta)$ inside the interval is higher than for any $\theta$ outside of the interval [73].

${ }^{8}$ Note that since in the latter case the LSP may be the gravitino, we do not exclude those points.
} 
mechanism, GUTs themselves "unify" the soft breaking parameters since they unify the different types of SM fermions in GUT representations. In SU(5) GUTs, for example, one is left with only two soft breaking mass matrices at the GUT scale per family, one for the sfermions in the five-dimensional matter representation and one for the sfermions in the ten-dimensional representation. In $\mathrm{SO}(10)$ GUTs, there is only one unified sfermion mass matrix. In addition, the symmetries of GUT flavour models like [34, 35, 43, 44] include various (non-Abelian) "family symmetries", which lead to hierarchical Yukawa matrices and impose (partially) universal soft breaking mass matrices among different generations. The combination of these effects can indeed lead to GUT scale boundary conditions close to the CMSSM. Finally, we like to note that the absence of deviations from the SM in flavour physics processes leads to constraints on flavour non-universalities in the SUSY spectrum (if the sparticles are not too heavy) and can be seen as an experimental hint that, if SUSY exists at a comparably low scale, it should indeed be close to flavour-universal. In any case, it will be interesting to see how the constraints on the SUSY spectrum change when the assumption of an exact CMSSM at the GUT scale is relaxed.

On the other hand, although we have analyzed here a specific example only, some of the key effects that lead to a predicted sparticle spectrum seem rather general, as long as the quark-lepton Yukawa ratios are predicted at the GUT scale together with (close-to) universal soft-breaking parameters (which we assume for the remainder of this discussion):

- The main reason for the predictions/constraints on the SUSY spectrum is the fact that (to our knowledge) all the possible sets of GUT predictions for the quark-lepton Yukawa ratios require a certain amount of SUSY threshold corrections for each generation. ${ }^{9}$ In general, to obtain the required size of the threshold corrections, one cannot have a sparticle spectrum which is too "split" (as e.g. in $[74,75]$ ), since otherwise the loop functions (cf. section 3) get too suppressed. More specifically, the required threshold corrections constrain the ratios of trilinear couplings, gaugino masses, $\mu$ and sfermion masses. In a CMSSM-like scenario, this means the ratios between $m_{0}, m_{1 / 2}$ and $A_{0}$ are constrained. Furthermore, since the most relevant threshold corrections are the ones which are $\tan \beta$-enhanced, $\tan \beta$ cannot bee too small.

- Finally, with the ratios between $m_{0}, m_{1 / 2}$ and $A_{0}$ constrained and a moderate to large value of $\tan \beta$, the measured value of the mass $m_{h}$ of the SM-like Higgs boson allows to constrain the SUSY scale. We note that this is an important ingredient, since the threshold corrections themselves depend only on the ratios of trilinear couplings, gaugino masses, $\mu$ and sfermion masses, and do not constrain the overall scale of the soft breaking parameters. The combination of the two effects leads to the result of a predicted sparticle spectrum from the assumed GUT boundary conditions.

Since $m_{h}$ plays an important role, we would like to comment that it would be highly desirable to have a more precise computation of the Higgs mass available for the "large stopmixing" regime. As discussed above, we use a theoretical uncertainty of $\pm 3 \mathrm{GeV}$, which

\footnotetext{
${ }^{9}$ One comment is in order here: in the CMSSM the SUSY threshold corrections are very similar for the first two families, and therefore the argument remains valid even if the quark-lepton Yukawa ratios are predicted for only two of the families, for the third family and either the second or the first family.
} 
is dominating the $1 \sigma$ interval for $m_{h}$ entering our fit. This theoretical uncertainty should, strictly speaking, not be treated on the same footing as a pure statistical uncertainty (but the same of course also holds true for systematic experimental uncertainties). Furthermore, there are indications that the theoretical uncertainty in the $m_{h}$ calculation in the most relevant regions of parameter space of our analysis may be larger, as recently discussed e.g. in [76], however there is no full agreement on this aspect. For our analysis we have used the external software FeynHiggs 2.11.3, the current version when our numerical analysis was performed, and the most commonly assumed estimate $\pm 3 \mathrm{GeV}$ for the theoretical uncertainty.

One may also ask why we did not emphasize the aspect of gauge coupling unification. From a bottom-up perspective, one could indeed conclude that in order to make the gauge couplings meet at high energy, the SUSY scale cannot be too high. However, in realistic GUTs, such statements are strongly affected by GUT threshold corrections. They can be implemented easily with REAP once the specific GUT model is known, however they are very model-dependent. In particular, for more general GUT-Higgs potentials it is known that gauge coupling unification is not resulting in a relevant constraint on the SUSY scale. For our analysis, we have therefore simply set the GUT scale to $2 \times 10^{16} \mathrm{GeV}$. In an explicit model, where the GUT threshold corrections can be computed and in particular if they significantly shift the GUT scale, we expect that the predictions for the SUSY spectrum will also be modified correspondingly, e.g. due to the increased amount of RG evolution.

Let us also comment on the fact that SusyTC is matching the MSSM to the SM at one common "SUSY scale". This is certainly a limitation of the current version of SusyTC. On the other hand, as discussed above, in the scenarios where SUSY threshold corrections play an important role, the sparticle spectrum cannot be too "split". This means that for the main applications of SusyTC, like the example presented in this section, one-step matching is sufficiently accurate.

\section{Summary and conclusion}

In this work we discussed how predictions for the sparticle spectrum can arise from GUTs, which feature predictions for the ratios of quark and lepton Yukawa couplings at high energy. To test them by comparing with the experimental data, the RG running between high and low energy has to be performed with sufficient accuracy, including threshold corrections. In SUSY theories, the one-loop threshold corrections when matching the SUSY model to the SM are of particular importance, since they can be enhanced by $\tan \beta$ or large trilinear couplings, and thus have the potential to strongly affect the quark-lepton mass relations. Since the SUSY threshold corrections depend on the SUSY parameters, they link a given GUT flavour model to the SUSY model. In other words, via the SUSY threshold corrections, GUT models can predict properties of the sparticle spectrum from the pattern of quark-lepton mass ratios at the GUT scale.

To accurately study such predictions, we extend and generalize various formulas in the literature which are needed for a precision analysis of SUSY flavour GUT models: for example, we extend the RGEs for the MSSM soft breaking parameters at two-loop by the 
additional terms in the seesaw type-I extension (cf. appendix A). We generalize the one-loop calculation of $\mu$ and pole mass calculation of $m_{A}$ and $m_{H^{+}}$to include inter-generational mixing in the self energies (cf. appendix B). Furthermore, we calculate the full one-loop SUSY threshold corrections for the down-type quark, up-type quark and charged lepton Yukawa coupling matrices in the electroweak unbroken phase (cf. section 3).

We introduce the new software tool SusyTC, a major extension to the Mathematica package REAP, where these formulas are implemented. In addition, SusyTC calculates the $\overline{\mathrm{DR}}$ sparticle spectrum and the SUSY scale $Q$, and can provide output in SLHA "Les Houches" files which are the necessary input for external software, e.g. for performing a twoloop Higgs mass calculation. REAP extended by SusyTC accepts general GUT scale Yukawa, trilinear and soft breaking mass matrices as well as non-universal gaugino masses as input, performs the RG evolution (integrating out the right-handed neutrinos at their mass thresholds in the type I seesaw extension of the MSSM) and automatically matches the MSSM to the SM, making it a convenient tool for top-down analyses of SUSY flavour GUT models.

We applied SusyTC to study the predictions for the parameters of the Constrained MSSM SUSY scenario from the set of GUT-scale Yukawa relations $\frac{y_{e}}{y_{d}}=-\frac{1}{2}, \frac{y_{\mu}}{y_{s}}=6$, and $\frac{y_{\tau}}{y_{b}}=-\frac{3}{2}$, which has been proposed recently in the context of GUT flavour models. With a Markov Chain Monte Carlo analysis we find a "best-fit" benchmark point as well as the $1 \sigma$ Bayesian confidence intervals for the sparticle masses. Without applying any constraints from LHC SUSY searches or dark matter, we find that the considered GUT scenario predicts a sparticle spectrum above past LHC sensitivities, but partly within reach of (a high-luminosity upgrade of) the LHC, and possibly fully testable at a future $\mathrm{O}(100 \mathrm{TeV})$ pp collider like the FCC-hh [80] or the SPPC [81].

\section{Acknowledgments}

We would like to thank Vinzenz Maurer for help with code optimisation and Christian Hohl for testing. We also thank Eros Cazzato, Thomas Hahn, Vinzenz Maurer, Stefano Orani and Sebastian Paßehr for useful discussions. This work has been supported by the Swiss National Science Foundation.

\section{A The $\beta$-functions in the seesaw type-I extension of the MSSM}

In this appendix we list the $\beta$-functions of the SUSY soft-breaking parameters in the MSSM extended by the additional terms in the seesaw type-I extension (obtained using the general formulas of [56]). Our conventions for $W$ and $\mathcal{L}_{\text {soft }}$ are given in (3.1) and (3.2).

\section{A.1 One-loop $\beta$-functions}

$$
\begin{aligned}
16 \pi^{2} \beta_{M_{1}} & =\frac{66}{5} g_{1}^{2} M_{1}, \\
16 \pi^{2} \beta_{M_{2}} & =2 g_{2}^{2} M_{2}, \\
16 \pi^{2} \beta_{M_{3}} & =-6 g_{3}^{2} M_{3},
\end{aligned}
$$




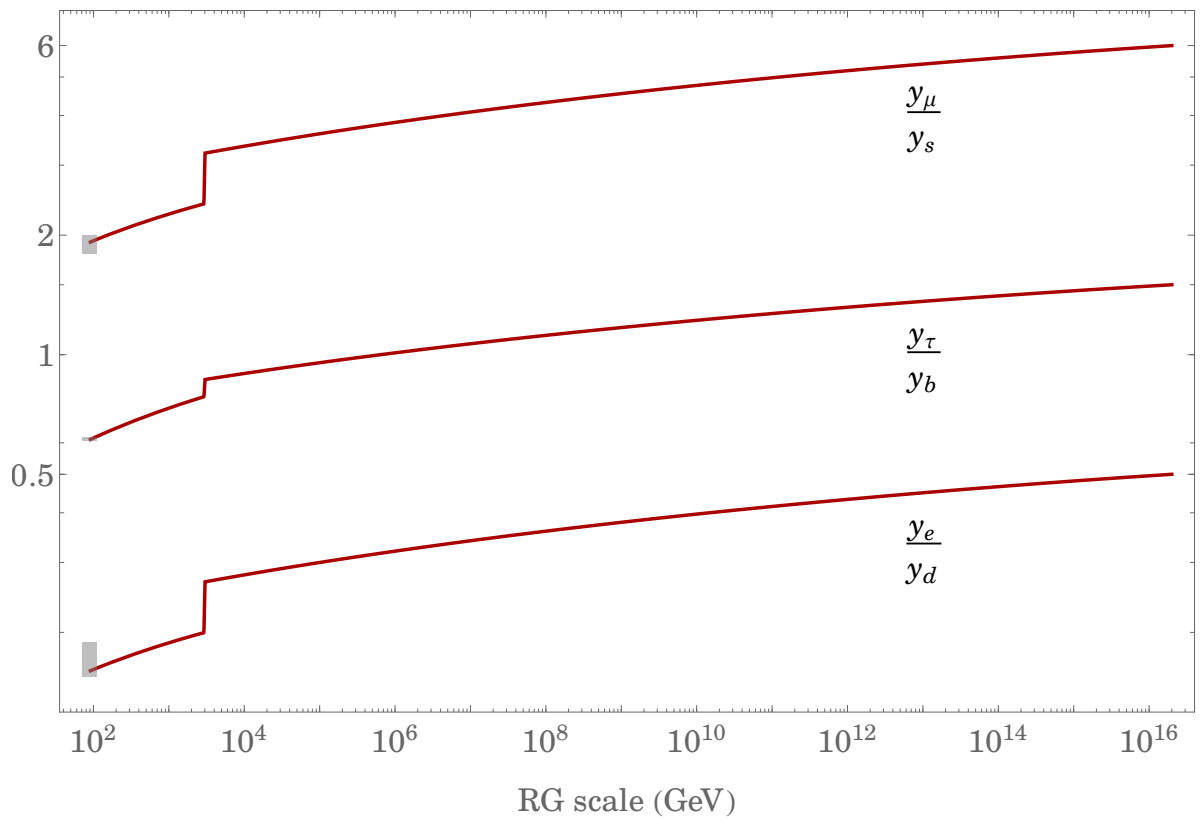

Figure 9. RG evolution of the Yukawa coupling ratios of the first, second and third family from the GUT-scale to the mass scale of the Z-boson. The GUT scale parameters correspond to our benchmark point from section 5 . The effects of the threshold corrections are clearly visible at the SUSY scale $Q=3015 \mathrm{GeV}$. The light gray areas indicate the experimental Yukawa coupling ratios at $M_{Z}$, taken from [36].

$$
\begin{aligned}
16 \pi^{2} \beta_{T_{u}}= & Y_{u}\left(2 Y_{d}^{\dagger} T_{d}+4 Y_{u}^{\dagger} T_{u}\right)+T_{u}\left(Y_{d}^{\dagger} Y_{d}+5 Y_{u}^{\dagger} Y_{u}\right) \\
& +Y_{u}\left(6 \operatorname{Tr}\left(Y_{u}^{\dagger} T_{u}\right)+2 \operatorname{Tr}\left(Y_{\nu}^{\dagger} T_{\nu}\right)+\frac{26}{15} g_{1}^{2} M_{1}+6 g_{2}^{2} M_{2}+\frac{32}{3} g_{3}^{2} M_{3}\right) \\
& +T_{u}\left(3 \operatorname{Tr}\left(Y_{u}^{\dagger} Y_{u}\right)+\operatorname{Tr}\left(Y_{\nu}^{\dagger} Y_{\nu}\right)-\frac{13}{15} g_{1}^{2}-3 g_{2}^{2}-\frac{16}{3} g_{3}^{2}\right), \\
16 \pi^{2} \beta_{T_{d}}= & Y_{d}\left(4 Y_{d}^{\dagger} T_{d}+2 Y_{u}^{\dagger} T_{u}\right)+T_{d}\left(5 Y_{d}^{\dagger} Y_{d}+Y_{u}^{\dagger} Y_{u}\right) \\
& +Y_{d}\left(6 \operatorname{Tr}\left(Y_{d}^{\dagger} T_{d}\right)+2 \operatorname{Tr}\left(Y_{e}^{\dagger} T_{e}\right)+\frac{14}{15} g_{1}^{2} M_{1}+6 g_{2}^{2} M_{2}+\frac{32}{3} g_{3}^{2} M_{3}\right) \\
& +T_{d}\left(3 \operatorname{Tr}\left(Y_{d}^{\dagger} Y_{d}\right)+\operatorname{Tr}\left(Y_{e}^{\dagger} Y_{e}\right)-\frac{7}{15} g_{1}^{2}-3 g_{2}^{2}-\frac{16}{3} g_{3}^{2}\right) \\
16 \pi^{2} \beta_{T_{e}}= & Y_{e}\left(4 Y_{e}^{\dagger} T_{e}+2 Y_{\nu}^{\dagger} T_{\nu}\right)+T_{e}\left(5 Y_{e}^{\dagger} Y_{e}+Y_{\nu}^{\dagger} Y_{\nu}\right) \\
& +Y_{e}\left(6 \operatorname{Tr}\left(Y_{d}^{\dagger} T_{d}\right)+2 \operatorname{Tr}\left(Y_{e}^{\dagger} T_{e}\right)+\frac{18}{5} g_{1}^{2} M_{1}+6 g_{2}^{2} M_{2}\right) \\
& +T_{e}\left(3 \operatorname{Tr}\left(Y_{d}^{\dagger} Y_{d}\right)+\operatorname{Tr}\left(Y_{e}^{\dagger} Y_{e}\right)-\frac{9}{5} g_{1}^{2}-3 g_{2}^{2}\right),
\end{aligned}
$$



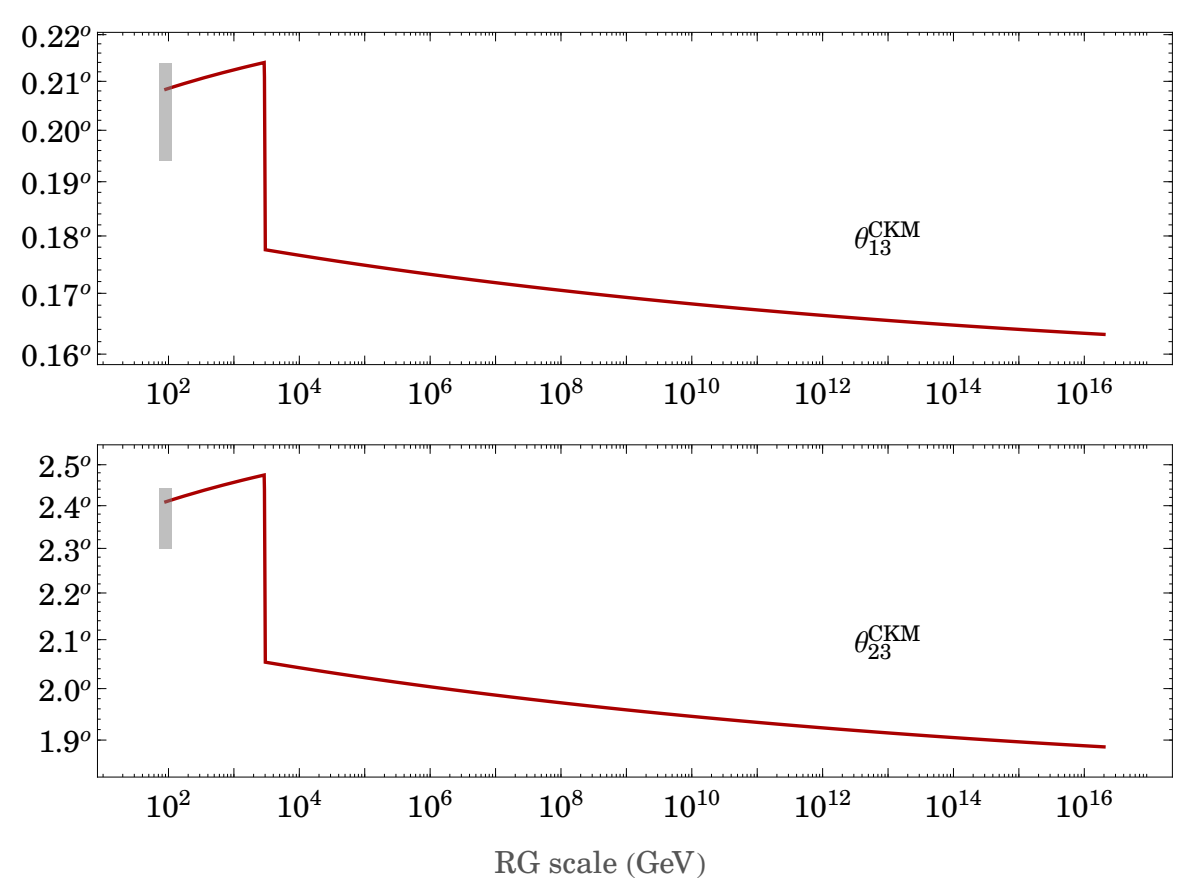

Figure 10. RG evolution of the CKM mixing angles $\theta_{13}^{\mathrm{CKM}}$ and $\theta_{23}^{\mathrm{CKM}}$ from the GUT-scale to the mass scale of the Z-boson. The GUT scale parameters correspond to our benchmark point from section 5. The effects of the threshold corrections are clearly visible at the SUSY scale $Q=3015 \mathrm{GeV}$. The light gray areas indicate the experimental values at $M_{Z}$.

$$
\begin{aligned}
16 \pi^{2} \beta_{T_{\nu}}= & Y_{\nu}\left(4 Y_{\nu}^{\dagger} T_{\nu}+2 Y_{e}^{\dagger} T_{e}\right)+T_{\nu}\left(5 Y_{\nu}^{\dagger} Y_{\nu}+Y_{e}^{\dagger} Y_{e}\right) \\
& +Y_{\nu}\left(6 \operatorname{Tr}\left(Y_{u}^{\dagger} T_{u}\right)+2 \operatorname{Tr}\left(Y_{\nu}^{\dagger} T_{\nu}\right)+\frac{6}{5} g_{1}^{2} M_{1}+6 g_{2}^{2} M_{2}\right) \\
& +T_{\nu}\left(3 \operatorname{Tr}\left(Y_{u}^{\dagger} Y_{u}\right)+\operatorname{Tr}\left(Y_{\nu}^{\dagger} Y_{\nu}\right)-\frac{3}{5} g_{1}^{2}-3 g_{2}^{2}\right), \\
16 \pi^{2} \beta_{m_{\tilde{L}}^{2}}= & 2 Y_{e}^{\dagger} m_{\tilde{e}}^{2} Y_{e}+2 Y_{\nu}^{\dagger} m_{\tilde{\nu}}^{2} Y_{\nu} \\
& +m_{\tilde{L}}^{2}\left(Y_{e}^{\dagger} Y_{e}+Y_{\nu}^{\dagger} Y_{\nu}\right)+\left(Y_{e}^{\dagger} Y_{e}+Y_{\nu}^{\dagger} Y_{\nu}\right) m_{\tilde{L}}^{2} \\
& +2 m_{H_{u}}^{2} Y_{\nu}^{\dagger} Y_{\nu}+2 m_{H_{d}}^{2} Y_{e}^{\dagger} Y_{e}+2 T_{e}^{\dagger} T_{e}+2 T_{\nu}^{\dagger} T_{\nu} \\
& -\frac{6}{5} g_{1}^{2}\left|M_{1}\right|^{2} \mathbf{1}_{3}-6 g_{2}^{2}|M 2|^{2} \mathbf{1}_{3}-\frac{3}{5} g_{1}^{2} S \mathbf{1}_{3}, \\
16 \pi^{2} \beta_{m_{\tilde{Q}}^{2}}= & 2 Y_{u}^{\dagger} m_{\tilde{u}}^{2} Y_{u}+2 Y_{d}^{\dagger} m_{\tilde{d}}^{2} Y_{d} \\
& +m_{\tilde{Q}}^{2}\left(Y_{u}^{\dagger} Y_{u}+Y_{d}^{\dagger} Y_{d}\right)+\left(Y_{u}^{\dagger} Y_{u}+Y_{d}^{\dagger} Y_{d}\right) m_{\tilde{Q}}^{2} \\
& +2 m_{H_{u}}^{2} Y_{u}^{\dagger} Y_{u}+2 m_{H_{d}}^{2} Y_{d}^{\dagger} Y_{d}+2 T_{u}^{\dagger} T_{u}+2 T_{d}^{\dagger} T_{d} \\
& -\frac{2}{15} g_{1}^{2}\left|M_{1}\right|^{2} \mathbf{1}_{3}-6 g_{2}^{2}|M 2|^{2} \mathbf{1}_{3}-\frac{32}{3} g_{3}^{2}\left|M_{3}\right|^{2} \mathbf{1}_{3}+\frac{1}{5} g_{1}^{2} S \mathbf{1}_{3},
\end{aligned}
$$




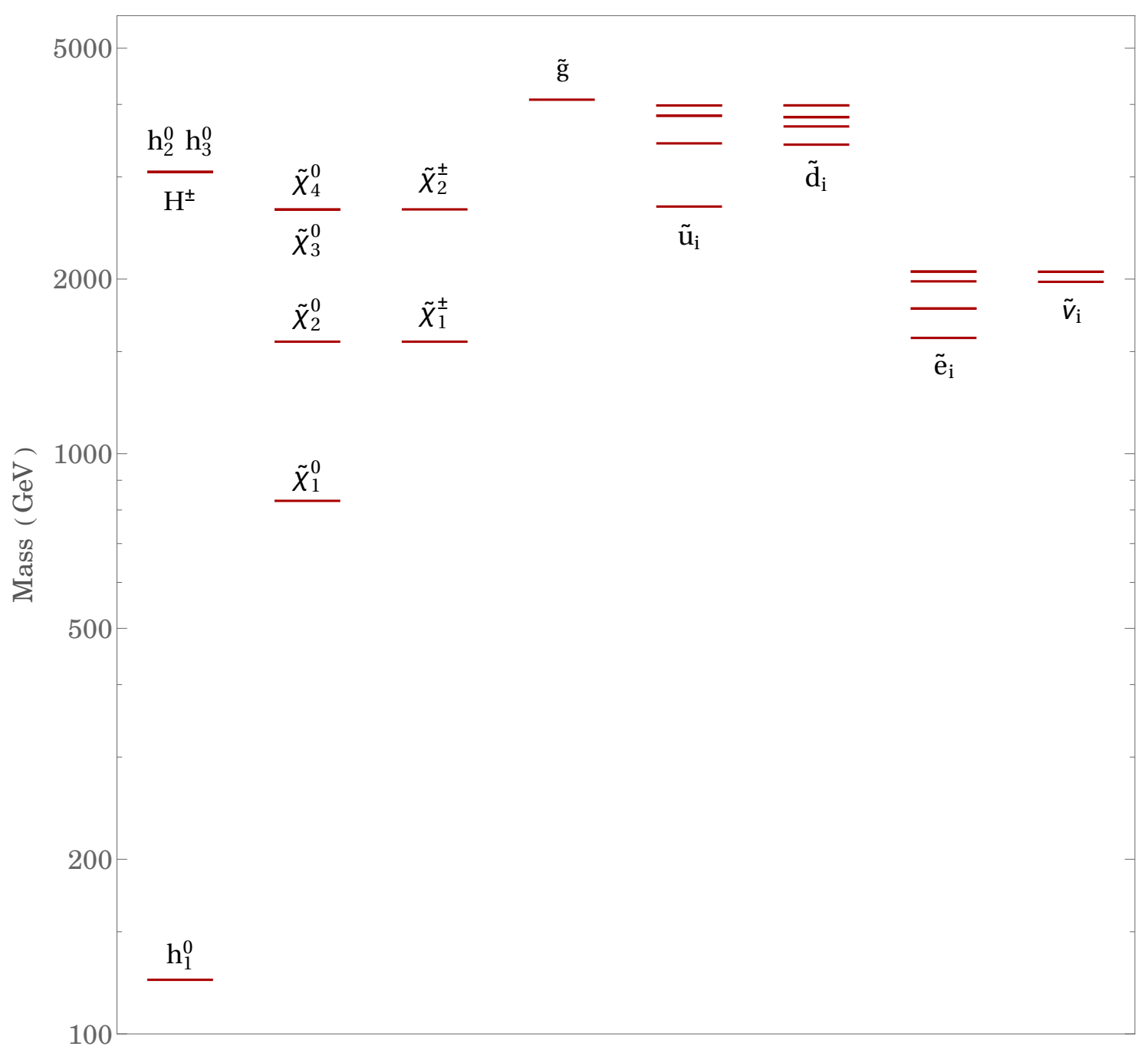

Figure 11. SUSY spectrum with SU(5) GUT scale boundary conditions $\frac{y_{e}}{y_{d}}=-\frac{1}{2}, \frac{y_{\mu}}{y_{s}}=6$, and $\frac{y_{\tau}}{y_{b}}=-\frac{3}{2}$, corresponding to our benchmark point from section 5 .

$$
\begin{aligned}
16 \pi^{2} \beta_{m_{\tilde{u}}^{2}}= & 4 Y_{u} m_{\tilde{Q}}^{2} Y_{u}^{\dagger}+2 Y_{u} Y_{u}^{\dagger} m_{\tilde{u}}^{2}+2 m_{\tilde{u}}^{2} Y_{u} Y_{u}^{\dagger} \\
& +4 m_{H_{u}}^{2} Y_{u} Y_{u}^{\dagger}+4 T_{u} T_{u}^{\dagger} \\
& -\frac{32}{15} g_{1}^{2}\left|M_{1}\right|^{2} \mathbf{1}_{3}-\frac{32}{3} g_{3}^{2}\left|M_{3}\right|^{2} \mathbf{1}_{3}-\frac{4}{5} g_{1}^{2} S \mathbf{1}_{3}, \\
16 \pi^{2} \beta_{m_{\tilde{d}}^{2}}= & 4 Y_{d} m_{\tilde{Q}}^{2} Y_{d}^{\dagger}+2 Y_{d} Y_{d}^{\dagger} m_{\tilde{d}}^{2}+2 m_{\tilde{d}}^{2} Y_{d} Y_{d}^{\dagger} \\
& +4 m_{H_{d}}^{2} Y_{d} Y_{d}^{\dagger}+4 T_{d} T_{d}^{\dagger} \\
& -\frac{8}{15} g_{1}^{2}\left|M_{1}\right|^{2} \mathbf{1}_{3}-\frac{32}{3} g_{3}^{2}\left|M_{3}\right|^{2} \mathbf{1}_{3}+\frac{2}{5} g_{1}^{2} S \mathbf{1}_{3},
\end{aligned}
$$




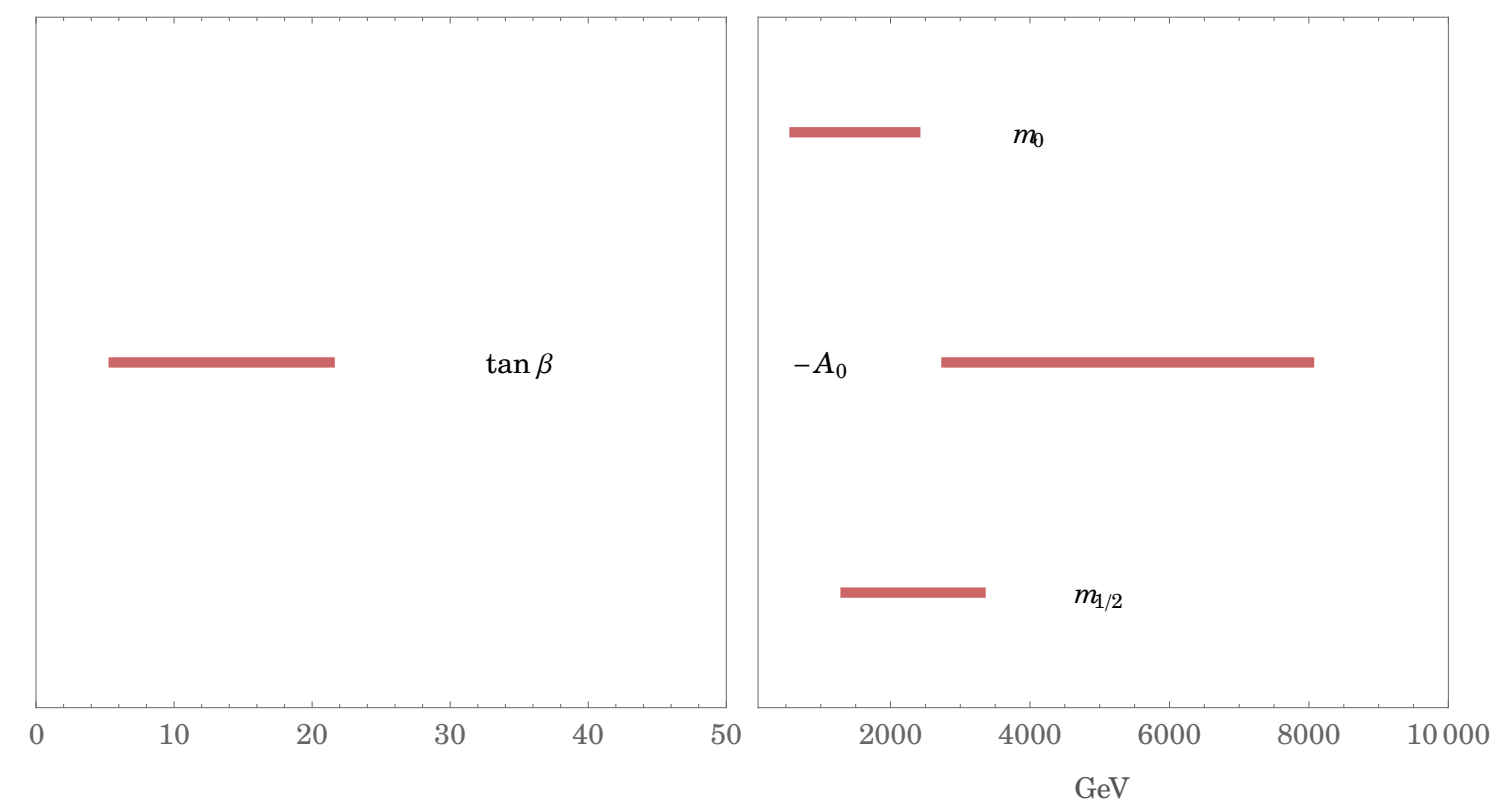

Figure 12. $1 \sigma$ HPD intervals for the Constrained MSSM soft-breaking parameters.

$$
\begin{aligned}
& 16 \pi^{2} \beta_{m_{\tilde{e}}^{2}=} 4 Y_{e} m_{\tilde{L}}^{2} Y_{e}^{\dagger}+2 Y_{e} Y_{e}^{\dagger} m_{\tilde{e}}^{2}+2 m_{\tilde{e}}^{2} Y_{e} Y_{e}^{\dagger} \\
&+4 m_{H_{d}}^{2} Y_{e} Y_{e}^{\dagger}+4 T_{e} T_{e}^{\dagger} \\
&-\frac{24}{5} g_{1}^{2}\left|M_{1}\right|^{2} \mathbf{1}_{3}+\frac{6}{5} g_{1}^{2} S \mathbf{1}_{3}, \\
& 16 \pi^{2} \beta_{m_{\tilde{\nu}}^{2}=} 4 Y_{\nu} m_{\tilde{L}}^{2} Y_{\nu}^{\dagger}+2 Y_{\nu} Y_{\nu}^{\dagger} m_{\tilde{\nu}}^{2}+2 m_{\tilde{\nu}}^{2} Y_{\nu} Y_{\nu}^{\dagger} \\
&+4 m_{H_{u}}^{2} Y_{\nu} Y_{\nu}^{\dagger}+4 T_{\nu} T_{\nu}^{\dagger}, \\
& 16 \pi^{2} \beta_{m_{H_{d}}^{2}}= 6 \operatorname{Tr}\left(Y_{d} m_{\tilde{Q}}^{2} Y_{d}^{\dagger}+Y_{d}^{\dagger} m_{\tilde{d}}^{2} Y d\right) \\
&+2 \operatorname{Tr}\left(Y_{e} m_{\tilde{L}}^{2} Y_{e}^{\dagger}+Y_{e}^{\dagger} m_{\tilde{e}}^{2} Y_{e}\right) \\
&+6 m_{H_{d}}^{2} \operatorname{Tr}\left(Y_{d}^{\dagger} Y_{d}\right)+2 m_{H_{d}}^{2} \operatorname{Tr}\left(Y_{e}^{\dagger} Y_{e}\right) \\
&+6 \operatorname{Tr}\left(T_{d}^{\dagger} T_{d}\right)+2 \operatorname{Tr}\left(T_{e}^{\dagger} T_{e}\right) \\
&-\frac{6}{5} g_{1}^{2}\left|M_{1}\right|^{2}-6 g_{2}^{2}\left|M_{2}\right|^{2}-\frac{3}{5} g_{1}^{2} S, \\
&= 6 \operatorname{Tr}\left(Y_{u} m_{\tilde{Q}}^{2} Y_{u}^{\dagger}+Y_{u}^{\dagger} m_{\tilde{u}}^{2} Y u\right) \\
&+2 \operatorname{Tr}\left(Y_{\nu} m_{\tilde{L}}^{2} Y_{\nu}^{\dagger}+Y_{\nu}^{\dagger} m_{\tilde{\nu}}^{2} Y_{\nu}\right) \\
&+6 m_{H_{u}}^{2} \operatorname{Tr}\left(Y_{u}^{\dagger} Y_{u}\right)+2 m_{H_{u}}^{2} \operatorname{Tr}\left(Y_{\nu}^{\dagger} Y_{\nu}\right) \\
&+6 \operatorname{Tr}\left(T_{u}^{\dagger} T_{u}\right)+2 \operatorname{Tr}\left(T_{\nu}^{\dagger} T_{\nu}\right) \\
&-\frac{6}{5} g_{1}^{2}\left|M_{1}\right|^{2}-6 g_{2}^{2}\left|M_{2}\right|^{2}+\frac{3}{5} g_{1}^{2} S, \\
& \beta_{m_{H_{u}}^{2}}
\end{aligned}
$$

with

$$
S=m_{H_{u}}^{2}-m_{H_{d}}^{2}+\operatorname{Tr}\left(m_{\tilde{Q}}^{2}-m_{\tilde{L}}^{2}+m_{\tilde{d}}^{2}+m_{\tilde{e}}^{2}-2 m_{\tilde{u}}^{2}\right)
$$




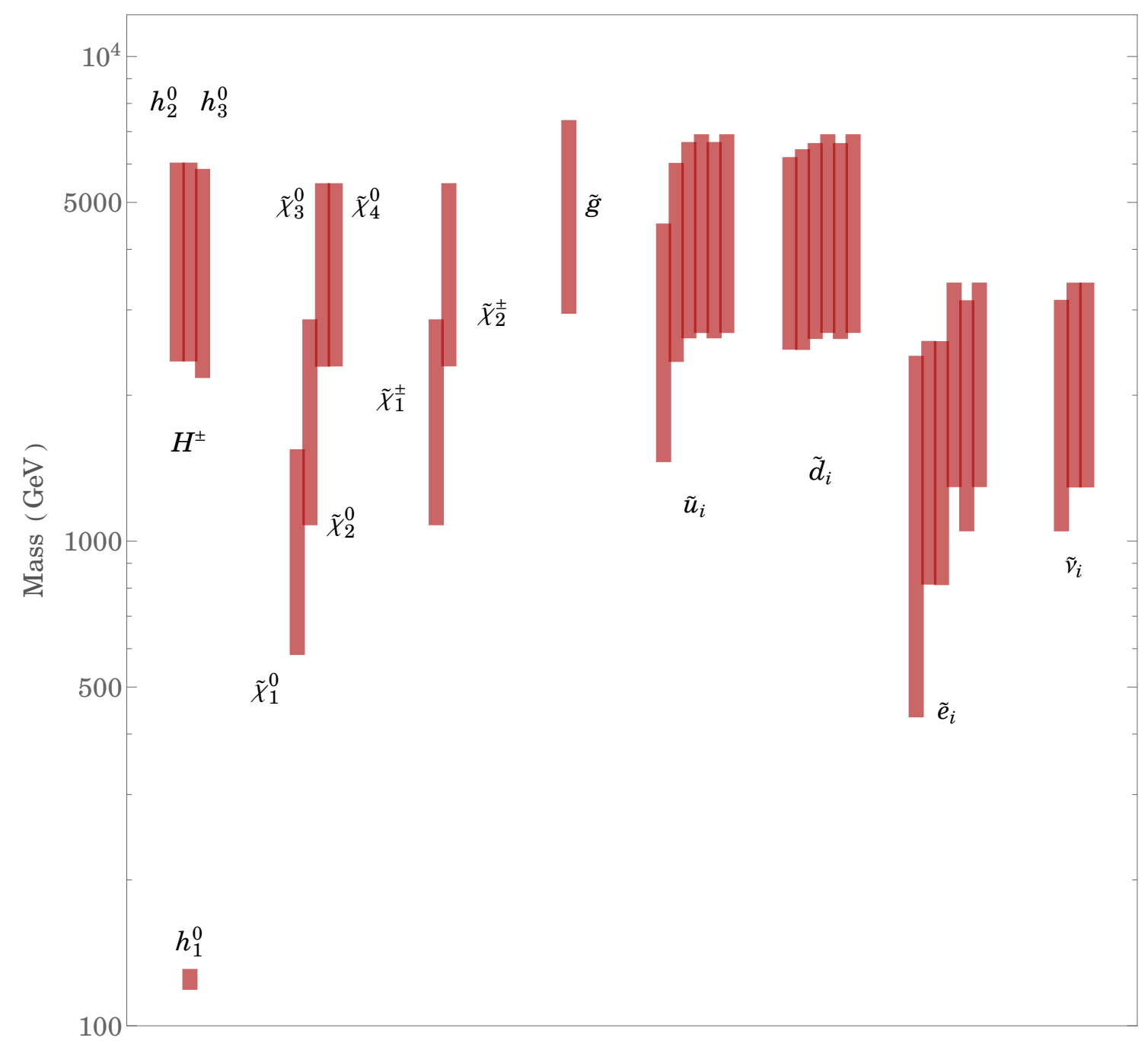

Figure 13. $1 \sigma$ HPD intervals for the sparticle spectrum and Higgs boson masses with SU(5) GUT scale boundary conditions $\frac{y_{e}}{y_{d}}=-\frac{1}{2}, \frac{y_{\mu}}{y_{s}}=6$, and $\frac{y_{\tau}}{y_{b}}=-\frac{3}{2}$. For about $70 \%$ of the data points, the LSP is the lightest neutralino $\tilde{\chi}_{1}^{0}$.

\section{A.2 Two-loop $\beta$-functions}

$$
\begin{aligned}
\left(16 \pi^{2}\right)^{2} \beta_{M_{1}}^{(2)}= & \frac{12}{5} g_{1}^{2} \operatorname{Tr}\left(T_{\nu} Y_{\nu}^{\dagger}\right)+\frac{28}{5} g_{1}^{2} \operatorname{Tr}\left(T_{d} Y_{d}^{\dagger}\right)+\frac{36}{5} g_{1}^{2} \operatorname{Tr}\left(T_{e} Y_{e}^{\dagger}\right)+\frac{52}{5} g_{1}^{2} \operatorname{Tr}\left(T_{u} Y_{u}^{\dagger}\right) \\
& -\frac{12}{5} g_{1}^{2} M_{1} \operatorname{Tr}\left(Y_{\nu} Y_{\nu}^{\dagger}\right)-\frac{28}{5} g_{1}^{2} M_{1} \operatorname{Tr}\left(Y_{d} Y_{d}^{\dagger}\right) \\
& -\frac{36}{5} g_{1}^{2} M_{1} \operatorname{Tr}\left(Y_{e} Y_{e}^{\dagger}\right)-\frac{52}{5} g_{1}^{2} M_{1} \operatorname{Tr}\left(Y_{u} Y_{u}^{\dagger}\right) \\
& +\frac{176}{5} g_{1}^{2} g_{3}^{2} M_{1}+\frac{176}{5} g_{1}^{2} g_{3}^{2} M_{3}+\frac{54}{5} g_{1}^{2} g_{2}^{2} M_{1}+\frac{54}{5} g_{1}^{2} g_{2}^{2} M_{2}+\frac{796}{25} g_{1}^{4} M_{1}, \\
\left(16 \pi^{2}\right)^{2} \beta_{M_{2}}^{(2)}= & 4 g_{2}^{2} \operatorname{Tr}\left(T_{\nu} Y_{\nu}^{\dagger}\right)+4 g_{2}^{2} \operatorname{Tr}\left(T_{e} Y_{e}^{\dagger}\right)+12 g_{2}^{2} \operatorname{Tr}\left(T_{d} Y_{d}^{\dagger}\right)+12 g_{2}^{2} \operatorname{Tr}\left(T_{u} Y_{u}^{\dagger}\right) \\
& -4 g_{2}^{2} M_{2} \operatorname{Tr}\left(Y_{\nu} Y_{\nu}^{\dagger}\right)-4 g_{2}^{2} M_{2} \operatorname{Tr}\left(Y_{e} Y_{e}^{\dagger}\right) \\
& -12 g_{2}^{2} M_{2} \operatorname{Tr}\left(Y_{u} Y_{u}^{\dagger}\right)-12 g_{2}^{2} M_{2} \operatorname{Tr}\left(Y_{d} Y_{d}^{\dagger}\right)
\end{aligned}
$$




$$
\begin{aligned}
& +\frac{18}{5} g_{1}^{2} g_{2}^{2} M_{1}+\frac{18}{5} g_{1}^{2} g_{3}^{2} M_{2}+48 g_{2}^{2} g_{3}^{2} M_{2}+48 g_{2}^{2} g_{3}^{2} M_{3}+100 g_{2}^{4} M_{2}, \\
& \left(16 \pi^{2}\right)^{2} \beta_{M_{3}}^{(2)}=8 g_{3}^{2} \operatorname{Tr}\left(T_{d} Y_{d}^{\dagger}\right)+8 g_{3}^{2} \operatorname{Tr}\left(T_{u} Y_{u}^{\dagger}\right)-8 g_{3}^{2} M_{3} \operatorname{Tr}\left(Y_{d} Y_{d}^{\dagger}\right)-8 g_{3}^{2} M_{3} \operatorname{Tr}\left(Y_{u} Y_{u}^{\dagger}\right) \\
& +\frac{22}{5} g_{1}^{2} g_{3}^{2} M_{1}+\frac{22}{5} g_{1}^{2} g_{3}^{2} M_{3}+18 g_{2}^{2} g_{3}^{2} M_{2}+18 g_{2}^{2} g_{3}^{2} M_{3}+56 g_{3}^{4} M_{3} \text {, } \\
& \left(16 \pi^{2}\right)^{2} \beta_{T_{u}}^{(2)}=-2 T_{u}\left(Y_{d}^{\dagger} Y_{d} Y_{d}^{\dagger} Y_{d}+2 Y_{d}^{\dagger} Y_{d} Y_{u}^{\dagger} Y_{u}+3 Y_{u}^{\dagger} Y_{u} Y_{u}^{\dagger} Y_{u}\right) \\
& -2 Y_{u}\left(2 Y_{d}^{\dagger} T_{d} Y_{d}^{\dagger} Y_{d}+2 Y_{d}^{\dagger} T_{d} Y_{u}^{\dagger} Y_{u}+2 Y_{d}^{\dagger} Y_{d} Y_{d}^{\dagger} T_{d}\right. \\
& \left.+Y_{d}^{\dagger} Y_{d} Y_{u}^{\dagger} T_{u}+4 Y_{u}^{\dagger} T_{u} Y_{u}^{\dagger} Y_{u}+3 Y_{u}^{\dagger} Y_{u} Y_{u}^{\dagger} T_{u}\right) \\
& -T_{u}\left(Y_{d}^{\dagger} Y_{d} \operatorname{Tr}\left(Y_{e} Y_{e}^{\dagger}+3 Y_{d} Y_{d}^{\dagger}\right)+5 Y_{u}^{\dagger} Y_{u} \operatorname{Tr}\left(Y_{\nu} Y_{\nu}^{\dagger}+3 Y_{u} Y_{u}^{\dagger}\right)\right) \\
& -2 Y_{u}\left(Y_{d}^{\dagger} T_{d} \operatorname{Tr}\left(Y_{e} Y_{e}^{\dagger}+3 Y_{d} Y_{d}^{\dagger}\right)+Y_{d}^{\dagger} Y_{d} \operatorname{Tr}\left(T_{e} Y_{e}^{\dagger}+3 T_{d} Y_{d}^{\dagger}\right)\right. \\
& \left.+2 Y_{u}^{\dagger} T_{u} \operatorname{Tr}\left(Y_{\nu} Y_{\nu}^{\dagger}+3 Y_{u} Y_{u}^{\dagger}\right)+3 Y_{u}^{\dagger} Y_{u} \operatorname{Tr}\left(T_{\nu} Y_{\nu}^{\dagger}+3 T_{u} Y_{u}^{\dagger}\right)\right) \\
& -T_{u}\left(\operatorname{Tr}\left(3 Y_{\nu} Y_{\nu}^{\dagger} Y_{\nu} Y_{\nu}^{\dagger}+Y_{\nu} Y_{e}^{\dagger} Y_{e} Y_{\nu}^{\dagger}\right)+3 \operatorname{Tr}\left(Y_{u} Y_{d}^{\dagger} Y_{d} Y_{u}^{\dagger}+3 Y_{u} Y_{u}^{\dagger} Y_{u} Y_{u}^{\dagger}\right)\right) \\
& -2 Y_{u}\left(\operatorname{Tr}\left(6 T_{\nu} Y_{\nu}^{\dagger} Y_{\nu} Y_{\nu}^{\dagger}+T_{\nu} Y_{e}^{\dagger} Y_{e} Y_{\nu}^{\dagger}+Y_{\nu} Y_{e}^{\dagger} T_{e} Y_{\nu}^{\dagger}\right)\right. \\
& \left.+3 \operatorname{Tr}\left(Y_{u} Y_{d}^{\dagger} T_{d} Y_{u}^{\dagger}+T_{u} Y_{d}^{\dagger} Y_{d} Y_{u}^{\dagger}+6 T_{u} Y_{u}^{\dagger} Y_{u} Y_{u}^{\dagger}\right)\right) \\
& +\frac{2}{5} g_{1}^{2}\left(T_{u} Y_{d}^{\dagger} Y_{d}+2 Y_{u} Y_{d}^{\dagger} T_{d}+3 Y_{u} Y_{u}^{\dagger} T_{u}-2 M_{1}\left(Y_{u} Y_{d}^{\dagger} Y_{d}+Y_{u} Y_{u}^{\dagger} Y_{u}\right)\right) \\
& +6 g_{2}^{2}\left(Y_{u} Y_{u}^{\dagger} T_{u}+2 T_{u} Y_{u}^{\dagger} Y_{u}-2 M_{2} Y_{u} Y_{u}^{\dagger} Y_{u}\right) \\
& +\left(16 g_{3}^{2}+\frac{4}{5} g_{1}^{2}\right)\left(2 Y_{u} \operatorname{Tr}\left(Y_{u}^{\dagger} T_{u}\right)+T_{u} \operatorname{Tr}\left(Y_{u}^{\dagger} Y_{u}\right)\right) \\
& +\frac{136}{45} g_{1}^{2} g_{3}^{2} T_{u}+\frac{15}{2} g_{2}^{4} T_{u}-\frac{16}{9} g_{3}^{4} T_{u}+\frac{2743}{450} g_{1}^{4} T_{u}+g_{1}^{2} g_{2}^{2} T_{u}+8 g_{2}^{2} g_{3}^{2} T_{u} \\
& -Y_{u} \operatorname{Tr}\left(Y_{u}^{\dagger} Y_{u}\right)\left(32 g_{3}^{2} M_{3}+\frac{8}{5} g_{1}^{2} M_{1}\right)-\frac{272}{45} g_{1}^{2} g_{3}^{2} Y_{u}\left(M_{1}+M_{3}\right) \\
& -2 g_{1}^{2} g_{2}^{2} Y_{u}\left(M_{1}+M_{2}\right)-16 g_{2}^{2} g_{3}^{2} Y_{u}\left(M_{2}+M_{3}\right) \\
& -30 g_{2}^{4} M_{2} Y_{u}-\frac{5486}{225} g_{1}^{4} M_{1} Y_{u}+\frac{64}{9} g_{3}^{4} M_{3} Y_{u}, \\
& \left(16 \pi^{2}\right)^{2} \beta_{T_{d}}^{(2)}=-2 T_{d}\left(3 Y_{d}^{\dagger} Y_{d} Y_{d}^{\dagger} Y_{d}+2 Y_{u}^{\dagger} Y_{u} Y_{d}^{\dagger} Y_{d}+Y_{u}^{\dagger} Y_{u} Y_{u}^{\dagger} Y_{u}\right) \\
& -2 Y_{d}\left(4 Y_{d}^{\dagger} T_{d} Y_{d}^{\dagger} Y_{d}+3 Y_{d}^{\dagger} Y_{d} Y_{d}^{\dagger} T_{d}+2 Y_{u}^{\dagger} T_{u} Y_{d}^{\dagger} Y_{d}\right. \\
& \left.+2 Y_{u}^{\dagger} T_{u} Y_{u}^{\dagger} Y_{u}+Y_{u}^{\dagger} Y_{u} Y_{d}^{\dagger} T_{d}+2 Y_{u}^{\dagger} Y_{u} Y_{u}^{\dagger} T_{u}\right) \\
& -T_{d}\left(5 Y_{d}^{\dagger} Y_{d} \operatorname{Tr}\left(Y_{e} Y_{e}^{\dagger}+3 Y_{d} Y_{d}^{\dagger}\right)+Y_{u}^{\dagger} Y_{u} \operatorname{Tr}\left(Y_{\nu} Y_{\nu}^{\dagger}+3 Y_{u} Y_{u}^{\dagger}\right)\right) \\
& -2 Y_{d}\left(2 Y_{d}^{\dagger} T_{d} \operatorname{Tr}\left(Y_{e} Y_{e}^{\dagger}+3 Y_{d} Y_{d}^{\dagger}\right)+3 Y_{d}^{\dagger} Y_{d} \operatorname{Tr}\left(T_{e} Y_{e}^{\dagger}+3 T_{d} Y_{d}^{\dagger}\right)\right. \\
& \left.+Y_{u}^{\dagger} T_{u} \operatorname{Tr}\left(Y_{\nu} Y_{\nu}^{\dagger}+3 Y_{u} Y_{u}^{\dagger}\right)+Y_{u}^{\dagger} Y_{u} \operatorname{Tr}\left(T_{\nu} Y_{\nu}^{\dagger}+3 T_{u} Y_{u}^{\dagger}\right)\right) \\
& -T_{d}\left(\operatorname{Tr}\left(Y_{e} Y_{\nu}^{\dagger} Y_{\nu} Y_{e}^{\dagger}+3 Y_{e} Y_{e}^{\dagger} Y_{e} Y_{e}^{\dagger}\right)+3 \operatorname{Tr}\left(Y_{u} Y_{d}^{\dagger} Y_{d} Y_{u}^{\dagger}+3 Y_{d} Y_{d}^{\dagger} Y_{d} Y_{d}^{\dagger}\right)\right) \\
& -2 Y_{d}\left(\operatorname{Tr}\left(Y_{e} Y_{\nu}^{\dagger} T_{\nu} Y_{e}^{\dagger}+T_{e} Y_{\nu}^{\dagger} Y_{\nu} Y_{e}^{\dagger}+6 T_{e} Y_{e}^{\dagger} Y_{e} Y_{e}^{\dagger}\right)\right. \\
& \left.+3 \operatorname{Tr}\left(T_{d} Y_{u}^{\dagger} Y_{u} Y_{d}^{\dagger}+T_{u} Y_{d}^{\dagger} Y_{d} Y_{u}^{\dagger}+6 T_{d} Y_{d}^{\dagger} Y_{d} Y_{d}^{\dagger}\right)\right)
\end{aligned}
$$




$$
\begin{aligned}
& +\frac{2}{5} g_{1}^{2}\left(2 T_{d} Y_{u}^{\dagger} Y_{u}+3 T_{d} Y_{d}^{\dagger} Y_{d}+3 Y_{d} Y_{d}^{\dagger} T_{d}+4 Y_{d} Y_{u}^{\dagger} T_{u}-4 M_{1}\left(Y_{d} Y_{d}^{\dagger} Y_{d}+Y_{d} Y_{u}^{\dagger} Y_{u}\right)\right) \\
& +6 g_{2}^{2}\left(Y_{d} Y_{d}^{\dagger} T_{d}+2 T_{d} Y_{d}^{\dagger} Y_{d}-2 M_{2} Y_{d} Y_{d}^{\dagger} Y_{d}\right) \\
& +\left(16 g_{3}^{2}-\frac{2}{5} g_{1}^{2}\right)\left(2 Y_{d} \operatorname{Tr}\left(Y_{d}^{\dagger} T_{d}\right)+T_{d} \operatorname{Tr}\left(Y_{d}^{\dagger} Y_{d}\right)\right) \\
& +\frac{6}{5} g_{1}^{2}\left(2 Y_{d} \operatorname{Tr}\left(Y_{e}^{\dagger} T_{e}\right)+T_{d} \operatorname{Tr}\left(Y_{e}^{\dagger} Y_{e}\right)\right) \\
& +\frac{8}{9} g_{1}^{2} g_{3}^{2} T_{d}+\frac{15}{2} g_{2}^{4} T_{d}-\frac{16}{9} g_{3}^{4} T_{d}+\frac{287}{90} g_{1}^{4} T_{d}+g_{1}^{2} g_{2}^{2} T_{d}+8 g_{2}^{2} g_{3}^{2} T_{d} \\
& -Y_{d} \operatorname{Tr}\left(Y_{d}^{\dagger} Y_{d}\right)\left(32 g_{3}^{2} M_{3}-\frac{4}{5} g_{1}^{2} M_{1}\right)-\frac{12}{5} g_{1}^{2} M_{1} Y_{d} \operatorname{Tr}\left(Y_{e}^{\dagger} Y_{e}\right) \\
& -\frac{16}{9} g_{1}^{2} g_{3}^{2} Y_{d}\left(M_{1}+M_{3}\right)-2 g_{1}^{2} g_{2}^{2} Y_{d}\left(M_{1}+M_{2}\right)-16 g_{2}^{2} g_{3}^{2} Y_{d}\left(M_{2}+M_{3}\right) \\
& \text { - } 30 g_{2}^{4} M_{2} Y_{d}-\frac{574}{45} g_{1}^{4} M_{1} Y_{d}+\frac{64}{9} g_{3}^{4} M_{3} Y_{d} \\
& \left(16 \pi^{2}\right)^{2} \beta_{T_{e}}^{(2)}=-2 T_{e}\left(3 Y_{e}^{\dagger} Y_{e} Y_{e}^{\dagger} Y_{e}+2 Y_{\nu}^{\dagger} Y_{\nu} Y_{e}^{\dagger} Y_{e}+Y_{\nu}^{\dagger} Y_{\nu} Y_{\nu}^{\dagger} Y_{\nu}\right) \\
& -2 Y_{e}\left(4 Y_{e}^{\dagger} T_{e} Y_{e}^{\dagger} Y_{e}+3 Y_{e}^{\dagger} Y_{e} Y_{e}^{\dagger} T_{e}+2 Y_{\nu}^{\dagger} T_{\nu} Y_{e}^{\dagger} Y_{e}\right. \\
& \left.+2 Y_{\nu}^{\dagger} T_{\nu} Y_{\nu}^{\dagger} Y_{\nu}+Y_{\nu}^{\dagger} Y_{\nu} Y_{e}^{\dagger} T_{e}+2 Y_{\nu}^{\dagger} Y_{\nu} Y_{\nu}^{\dagger} T_{\nu}\right) \\
& -T_{e}\left(5 Y_{e}^{\dagger} Y_{e} \operatorname{Tr}\left(Y_{e} Y_{e}^{\dagger}+3 Y_{d} Y_{d}^{\dagger}\right)+Y_{\nu}^{\dagger} Y_{\nu} \operatorname{Tr}\left(Y_{\nu} Y_{\nu}^{\dagger}+3 Y_{u} Y_{u}^{\dagger}\right)\right) \\
& -2 Y_{e}\left(2 Y_{e}^{\dagger} T_{e} \operatorname{Tr}\left(Y_{e} Y_{e}^{\dagger}+3 Y_{d} Y_{d}^{\dagger}\right)+3 Y_{e}^{\dagger} Y_{e} \operatorname{Tr}\left(T_{e} Y_{e}^{\dagger}+3 T_{d} Y_{d}^{\dagger}\right)\right. \\
& \left.+Y_{\nu}^{\dagger} T_{\nu} \operatorname{Tr}\left(Y_{\nu} Y_{\nu}^{\dagger}+3 Y_{u} Y_{u}^{\dagger}\right)+Y_{\nu}^{\dagger} Y_{\nu} \operatorname{Tr}\left(T_{\nu} Y_{\nu}^{\dagger}+3 T_{u} Y_{u}^{\dagger}\right)\right) \\
& -T_{e}\left(\operatorname{Tr}\left(Y_{e} Y_{\nu}^{\dagger} Y_{\nu} Y_{e}^{\dagger}+3 Y_{e} Y_{e}^{\dagger} Y_{e} Y_{e}^{\dagger}\right)+3 \operatorname{Tr}\left(Y_{d} Y_{u}^{\dagger} Y_{u} Y_{d}^{\dagger}+3 Y_{d} Y_{d}^{\dagger} Y_{d} Y_{d}^{\dagger}\right)\right) \\
& -2 Y_{e}\left(\operatorname{Tr}\left(Y_{e} Y_{\nu}^{\dagger} T_{\nu} Y_{e}^{\dagger}+T_{e} Y_{\nu}^{\dagger} Y_{\nu} Y_{e}^{\dagger}+6 T_{e} Y_{e}^{\dagger} Y_{e} Y_{e}^{\dagger}\right)\right. \\
& \left.+3 \operatorname{Tr}\left(T_{d} Y_{u}^{\dagger} Y_{u} Y_{d}^{\dagger}+T_{u} Y_{d}^{\dagger} Y_{d} Y_{u}^{\dagger}+6 T_{d} Y_{d}^{\dagger} Y_{d} Y_{d}^{\dagger}\right)\right) \\
& +\frac{6}{5} g_{1}^{2}\left(Y_{e} Y_{e}^{\dagger} T_{e}-T_{e} Y_{e}^{\dagger} Y_{e}\right) \\
& +6 g_{2}^{2}\left(Y_{e} Y_{e}^{\dagger} T_{e}+2 T_{e} Y_{e}^{\dagger} Y_{e}-2 M_{2} Y_{e} Y_{e}^{\dagger} Y_{e}\right) \\
& +\left(16 g_{3}^{2}-\frac{2}{5} g_{1}^{2}\right)\left(2 Y_{e} \operatorname{Tr}\left(Y_{d}^{\dagger} T_{d}\right)+T_{e} \operatorname{Tr}\left(Y_{d}^{\dagger} Y_{d}\right)\right) \\
& +\frac{6}{5} g_{1}^{2}\left(2 Y_{e} \operatorname{Tr}\left(Y_{e}^{\dagger} T_{e}\right)+T_{e} \operatorname{Tr}\left(Y_{e}^{\dagger} Y_{e}\right)\right) \\
& +\frac{15}{2} g_{2}^{4} T_{e}+\frac{27}{2} g_{1}^{4} T_{e}+\frac{9}{5} g_{1}^{2} g_{2}^{2} T_{e} \\
& -Y_{e} \operatorname{Tr}\left(Y_{d}^{\dagger} Y_{d}\right)\left(32 g_{3}^{2} M_{3}-\frac{4}{5} g_{1}^{2} M_{1}\right)-\frac{12}{5} g_{1}^{2} M_{1} Y_{e} \operatorname{Tr}\left(Y_{e}^{\dagger} Y_{e}\right) \\
& -\frac{18}{5} g_{1}^{2} g_{2}^{2} Y_{d}\left(M_{1}+M_{2}\right)-30 g_{2}^{4} M_{2} Y_{e}-54 g_{1}^{4} M_{1} Y_{e} \text {, } \\
& \left(16 \pi^{2}\right)^{2} \beta_{T_{\nu}}^{(2)}=-2 T_{\nu}\left(Y_{e}^{\dagger} Y_{e} Y_{e}^{\dagger} Y_{e}+2 Y_{e}^{\dagger} Y_{e} Y_{\nu}^{\dagger} Y_{\nu}+3 Y_{\nu}^{\dagger} Y_{\nu} Y_{\nu}^{\dagger} Y_{\nu}\right) \\
& -2 Y_{\nu}\left(2 Y_{e}^{\dagger} T_{e} Y_{e}^{\dagger} Y_{e}+2 Y_{e}^{\dagger} T_{e} Y_{\nu}^{\dagger} Y_{\nu}+2 Y_{e}^{\dagger} Y_{e} Y_{e}^{\dagger} T_{e}\right. \\
& \left.+Y_{e}^{\dagger} Y_{e} Y_{\nu}^{\dagger} T_{\nu}+4 Y_{\nu}^{\dagger} T_{\nu} Y_{\nu}^{\dagger} Y_{\nu}+3 Y_{\nu}^{\dagger} Y_{\nu} Y_{\nu}^{\dagger} T_{\nu}\right)
\end{aligned}
$$




$$
\begin{aligned}
& -T_{\nu}\left(Y_{e}^{\dagger} Y_{e} \operatorname{Tr}\left(Y_{e} Y_{e}^{\dagger}+3 Y_{d} Y_{d}^{\dagger}\right)+5 Y_{\nu}^{\dagger} Y_{\nu} \operatorname{Tr}\left(Y_{\nu} Y_{\nu}^{\dagger}+3 Y_{u} Y_{u}^{\dagger}\right)\right) \\
& -2 Y_{\nu}\left(Y_{e}^{\dagger} T_{e} \operatorname{Tr}\left(Y_{e} Y_{e}^{\dagger}+3 Y_{d} Y_{d}^{\dagger}\right)+Y_{e}^{\dagger} Y_{e} \operatorname{Tr}\left(T_{e} Y_{e}^{\dagger}+3 T_{d} Y_{d}^{\dagger}\right)\right. \\
& \left.+2 Y_{\nu}^{\dagger} T_{\nu} \operatorname{Tr}\left(Y_{\nu} Y_{\nu}^{\dagger}+3 Y_{u} Y_{u}^{\dagger}\right)+3 Y_{\nu}^{\dagger} Y_{\nu} \operatorname{Tr}\left(T_{\nu} Y_{\nu}^{\dagger}+3 T_{u} Y_{u}^{\dagger}\right)\right) \\
& -T_{\nu}\left(\operatorname{Tr}\left(3 Y_{\nu} Y_{\nu}^{\dagger} Y_{\nu} Y_{\nu}^{\dagger}+Y_{\nu} Y_{e}^{\dagger} Y_{e} Y_{\nu}^{\dagger}\right)+3 \operatorname{Tr}\left(Y_{u} Y_{d}^{\dagger} Y_{d} Y_{u}^{\dagger}+3 Y_{u} Y_{u}^{\dagger} Y_{u} Y_{u}^{\dagger}\right)\right) \\
& -2 Y_{\nu}\left(\operatorname{Tr}\left(T_{\nu} Y_{e}^{\dagger} Y_{e} Y_{\nu}^{\dagger}+Y_{\nu} Y_{e}^{\dagger} T_{e} Y_{\nu}^{\dagger}+6 T_{\nu} Y_{\nu}^{\dagger} Y_{\nu} Y_{\nu}^{\dagger}\right)\right. \\
& \left.+3 \operatorname{Tr}\left(T_{d} Y_{u}^{\dagger} Y_{u} Y_{d}^{\dagger}+T_{u} Y_{d}^{\dagger} Y_{d} Y_{u}^{\dagger}+6 T_{u} Y_{u}^{\dagger} Y_{u} Y_{u}^{\dagger}\right)\right) \\
& +\frac{6}{5} g_{1}^{2}\left(2 T_{\nu} Y_{\nu}^{\dagger} Y_{\nu}+2 Y_{\nu} Y_{e}^{\dagger} T_{e}+Y_{\nu} Y_{\nu}^{\dagger} T_{\nu}+T_{\nu} Y_{e}^{\dagger} Y_{e}-2 M_{1}\left(Y_{\nu} Y_{\nu}^{\dagger} Y_{\nu}+Y_{\nu} Y_{e}^{\dagger} Y_{e}\right)\right) \\
& +6 g_{2}^{2}\left(Y_{\nu} Y_{\nu}^{\dagger} T_{\nu}+2 T_{\nu} Y_{\nu}^{\dagger} Y_{\nu}-2 M_{2} Y_{\nu} Y_{\nu}^{\dagger} Y_{\nu}\right) \\
& +\left(16 g_{3}^{2}+\frac{4}{5} g_{1}^{2}\right)\left(2 Y_{\nu} \operatorname{Tr}\left(Y_{u}^{\dagger} T_{u}\right)+T_{\nu} \operatorname{Tr}\left(Y_{u}^{\dagger} Y_{u}\right)\right) \\
& +\frac{15}{2} g_{2}^{4} T_{\nu}+\frac{207}{50} g_{1}^{4} T_{\nu}+\frac{9}{5} g_{1}^{2} g_{2}^{2} T_{\nu} \\
& -Y_{\nu} \operatorname{Tr}\left(Y_{u}^{\dagger} Y_{u}\right)\left(32 g_{3}^{2} M_{3}+\frac{8}{5} g_{1}^{2} M_{1}\right) \\
& -\frac{18}{5} g_{1}^{2} g_{2}^{2} Y_{\nu}\left(M_{1}+M_{2}\right)-30 g_{2}^{4} M_{2} Y_{\nu}-\frac{414}{25} g_{1}^{4} M_{1} Y_{\nu}, \\
& \left(16 \pi^{2}\right)^{2} \beta_{m_{\tilde{L}}^{2}}^{(2)}=-4 T_{e}^{\dagger}\left(T_{e} Y_{e}^{\dagger} Y_{e}+Y_{e} Y_{e}^{\dagger} T_{e}\right)-4 T_{\nu}^{\dagger}\left(T_{\nu} Y_{\nu}^{\dagger} Y_{\nu}+Y_{\nu} Y_{\nu}^{\dagger} T_{\nu}\right) \\
& -2 Y_{e}^{\dagger}\left(2 m_{\tilde{e}}^{2} Y_{e} Y_{e}^{\dagger} Y_{e}+2 T_{e} T_{e}^{\dagger} Y_{e}+2 Y_{e} T_{e}^{\dagger} T_{e}\right. \\
& \left.+2 Y_{e} Y_{e}^{\dagger} m_{\tilde{e}}^{2} Y_{e}+Y_{e} Y_{e}^{\dagger} Y_{e} m_{\tilde{L}}^{2}+2 Y_{e} m_{\tilde{L}}^{2} Y_{e}^{\dagger} Y_{e}\right) \\
& -2 Y_{\nu}^{\dagger}\left(2 m_{\tilde{\nu}}^{2} Y_{\nu} Y_{\nu}^{\dagger} Y_{\nu}+2 T_{\nu} T_{\nu}^{\dagger} Y_{\nu}+2 Y_{\nu} T_{\nu}^{\dagger} T_{\nu}\right. \\
& \left.+2 Y_{\nu} Y_{\nu}^{\dagger} m_{\tilde{\nu}}^{2} Y_{\nu}+Y_{\nu} Y_{\nu}^{\dagger} Y_{\nu} m_{\tilde{L}}^{2}+2 Y_{\nu} m_{\tilde{L}}^{2} Y_{\nu}^{\dagger} Y_{\nu}\right) \\
& -2\left(4 m_{H_{d}}^{2}+m_{\tilde{L}}^{2}\right) Y_{e}^{\dagger} Y_{e} Y_{e}^{\dagger} Y_{e}-2\left(4 m_{H_{u}}^{2}+m_{\tilde{L}}^{2}\right) Y_{\nu}^{\dagger} Y_{\nu} Y_{\nu}^{\dagger} Y_{\nu} \\
& -2 T_{e}^{\dagger}\left(T_{e} \operatorname{Tr}\left(Y_{e} Y_{e}^{\dagger}+3 Y_{d} Y_{d}^{\dagger}\right)+Y_{e} \operatorname{Tr}\left(T_{e} Y_{e}^{\dagger}+3 T_{d} Y_{d}^{\dagger}\right)\right) \\
& -2 T_{\nu}^{\dagger}\left(T_{\nu} \operatorname{Tr}\left(Y_{\nu} Y_{\nu}^{\dagger}+3 Y_{u} Y_{u}^{\dagger}\right)+Y_{\nu} \operatorname{Tr}\left(T_{\nu} Y_{\nu}^{\dagger}+3 T_{u} Y_{u}^{\dagger}\right)\right) \\
& -Y_{e}^{\dagger}\left(2 m_{\tilde{e}}^{2} Y_{e} \operatorname{Tr}\left(Y_{e} Y_{e}^{\dagger}+3 Y_{d} Y_{d}^{\dagger}\right)+2 T_{e} \operatorname{Tr}\left(Y_{e} T_{e}^{\dagger}+3 Y_{d} T_{d}^{\dagger}\right)\right. \\
& \left.+2 Y_{e} \operatorname{Tr}\left(T_{e} T_{e}^{\dagger}+3 T_{d} T_{d}^{\dagger}\right)+Y_{e} m_{\tilde{L}}^{2} \operatorname{Tr}\left(Y_{e} Y_{e}^{\dagger}+3 Y_{d} Y_{d}^{\dagger}\right)\right) \\
& -Y_{\nu}^{\dagger}\left(2 m_{\tilde{\nu}}^{2} Y_{\nu} \operatorname{Tr}\left(Y_{\nu} Y_{\nu}^{\dagger}+3 Y_{u} Y_{u}^{\dagger}\right)+2 T_{\nu} \operatorname{Tr}\left(Y_{\nu} T_{\nu}^{\dagger}+3 Y_{u} T_{u}^{\dagger}\right)\right. \\
& \left.+2 Y_{\nu} \operatorname{Tr}\left(T_{\nu} T_{\nu}^{\dagger}+3 T_{u} T_{u}^{\dagger}\right)+Y_{\nu} m_{\tilde{L}}^{2} \operatorname{Tr}\left(Y_{\nu} Y_{\nu}^{\dagger}+3 Y_{u} Y_{u}^{\dagger}\right)\right) \\
& \text { - }\left(4 m_{H_{d}}^{2}+m_{\tilde{L}}^{2}\right) Y_{e}^{\dagger} Y_{e} \operatorname{Tr}\left(Y_{e} Y_{e}^{\dagger}+3 Y_{d} Y_{d}^{\dagger}\right) \\
& \text { - }\left(4 m_{H_{u}}^{2}+m_{\tilde{L}}^{2}\right) Y_{\nu}^{\dagger} Y_{\nu} \operatorname{Tr}\left(Y_{\nu} Y_{\nu}^{\dagger}+3 Y_{u} Y_{u}^{\dagger}\right) \\
& -2 Y_{e}^{\dagger} Y_{e} \operatorname{Tr}\left(Y_{e} m_{\tilde{L}}^{2} Y_{e}^{\dagger}+Y_{e}^{\dagger} m_{\tilde{e}}^{2} Y_{e}+3 Y_{d} m_{\tilde{Q}}^{2} Y_{d}^{\dagger}+3 Y_{d}^{\dagger} m_{\tilde{d}}^{2} Y_{d}\right) \\
& -2 Y_{\nu}^{\dagger} Y_{\nu} \operatorname{Tr}\left(Y_{\nu} m_{\tilde{L}}^{2} Y_{\nu}^{\dagger}+Y_{\nu}^{\dagger} m_{\tilde{\nu}}^{2} Y_{\nu}+3 Y_{u} m_{\tilde{Q}}^{2} Y_{u}^{\dagger}+3 Y_{u}^{\dagger} m_{\tilde{u}}^{2} Y_{u}\right) \\
& +\frac{6}{5} g_{1}^{2}\left(2 m_{H_{d}}^{2} Y_{e}^{\dagger} Y_{e}+m_{\tilde{L}}^{2} Y_{e}^{\dagger} Y_{e}+Y_{e}^{\dagger} Y_{e} m_{\tilde{L}}^{2}+2 Y_{e}^{\dagger} m_{\tilde{e}}^{2} Y_{e}+2 T_{e}^{\dagger} T_{e}\right)
\end{aligned}
$$




$$
\begin{aligned}
& -\frac{12}{5} g_{1}^{2}\left(M_{1}^{*} Y_{e}^{\dagger} T_{e}-2\left|M_{1}\right|^{2} Y_{e}^{\dagger} Y_{e}+M_{1} T_{e}^{\dagger} Y_{e}\right) \\
& +\frac{621}{25} g_{1}^{4}\left|M_{1}\right|^{2} \mathbf{1}_{3}+\frac{18}{5} g_{1}^{2} g_{2}^{2}\left(\left|M_{1}\right|^{2}+\left|M_{2}\right|^{2}\right) \mathbf{1}_{3} \\
& +\frac{18}{5} g_{1}^{2} g_{2}^{2} \operatorname{Re}\left(M_{1} M_{2}^{*}\right) \mathbf{1}_{3}+33 g_{2}^{4}\left|M_{2}\right|^{2} \mathbf{1}_{3} \\
& +\frac{3}{5} g_{1}^{2} \sigma_{1} \mathbf{1}_{3}+3 g_{2}^{2} \sigma_{2} \mathbf{1}_{3}-\frac{6}{5} g_{1}^{2} S^{\prime} \mathbf{1}_{3}, \\
& \left(16 \pi^{2}\right)^{2} \beta_{m_{\mathscr{Q}}^{2}}^{(2)}=-4 T_{d}^{\dagger}\left(T_{d} Y_{d}^{\dagger} Y_{d}+Y_{d} Y_{d}^{\dagger} T_{d}\right)-4 T_{u}^{\dagger}\left(T_{u} Y_{u}^{\dagger} Y_{u}+Y_{u} Y_{u}^{\dagger} T_{u}\right) \\
& -2 Y_{d}^{\dagger}\left(2 m_{\tilde{d}}^{2} Y_{d} Y_{d}^{\dagger} Y_{d}+2 T_{d} T_{d}^{\dagger} Y_{d}+2 Y_{d} T_{d}^{\dagger} T_{d}\right. \\
& \left.+2 Y_{d} Y_{d}^{\dagger} m_{\tilde{d}}^{2} Y_{d}+Y_{d} Y_{d}^{\dagger} Y_{d} m_{\tilde{Q}}^{2}+2 Y_{d} m_{\tilde{Q}}^{2} Y_{d}^{\dagger} Y_{d}\right) \\
& -2 Y_{u}^{\dagger}\left(2 m_{\tilde{u}}^{2} Y_{u} Y_{u}^{\dagger} Y_{u}+2 T_{u} T_{u}^{\dagger} Y_{u}+2 Y_{u} T_{u}^{\dagger} T_{u}\right. \\
& \left.+2 Y_{u} Y_{u}^{\dagger} m_{\tilde{u}}^{2} Y_{u}+Y_{u} Y_{u}^{\dagger} Y_{u} m_{\tilde{Q}}^{2}+2 Y_{u} m_{\tilde{Q}}^{2} Y_{u}^{\dagger} Y_{u}\right) \\
& -2\left(4 m_{H_{d}}^{2}+m_{\tilde{Q}}^{2}\right) Y_{d}^{\dagger} Y_{d} Y_{d}^{\dagger} Y_{d}-2\left(4 m_{H_{u}}^{2}+m_{\tilde{Q}}^{2}\right) Y_{u}^{\dagger} Y_{u} Y_{u}^{\dagger} Y_{u} \\
& -2 T_{d}^{\dagger}\left(T_{d} \operatorname{Tr}\left(Y_{e} Y_{e}^{\dagger}+3 Y_{d} Y_{d}^{\dagger}\right)+Y_{d} \operatorname{Tr}\left(T_{e} Y_{e}^{\dagger}+3 T_{d} Y_{d}^{\dagger}\right)\right) \\
& -2 T_{u}^{\dagger}\left(T_{u} \operatorname{Tr}\left(Y_{\nu} Y_{\nu}^{\dagger}+3 Y_{u} Y_{u}^{\dagger}\right)+Y_{u} \operatorname{Tr}\left(T_{\nu} Y_{\nu}^{\dagger}+3 T_{u} Y_{u}^{\dagger}\right)\right) \\
& -Y_{d}^{\dagger}\left(2 m_{\tilde{d}}^{2} Y_{d} \operatorname{Tr}\left(Y_{e} Y_{e}^{\dagger}+3 Y_{d} Y_{d}^{\dagger}\right)+2 T_{d} \operatorname{Tr}\left(Y_{e} T_{e}^{\dagger}+3 Y_{d} T_{d}^{\dagger}\right)\right. \\
& \left.+2 Y_{d} \operatorname{Tr}\left(T_{e} T_{e}^{\dagger}+3 T_{d} T_{d}^{\dagger}\right)+Y_{d} m_{\tilde{Q}}^{2} \operatorname{Tr}\left(Y_{e} Y_{e}^{\dagger}+3 Y_{d} Y_{d}^{\dagger}\right)\right) \\
& -Y_{u}^{\dagger}\left(2 m_{\tilde{u}}^{2} Y_{u} \operatorname{Tr}\left(Y_{\nu} Y_{\nu}^{\dagger}+3 Y_{u} Y_{u}^{\dagger}\right)+2 T_{u} \operatorname{Tr}\left(Y_{\nu} T_{\nu}^{\dagger}+3 Y_{u} T_{u}^{\dagger}\right)\right. \\
& \left.+2 Y_{u} \operatorname{Tr}\left(T_{\nu} T_{\nu}^{\dagger}+3 T_{u} T_{u}^{\dagger}\right)+Y_{u} m_{\tilde{Q}}^{2} \operatorname{Tr}\left(Y_{\nu} Y_{\nu}^{\dagger}+3 Y_{u} Y_{u}^{\dagger}\right)\right) \\
& -\left(4 m_{H_{d}}^{2}+m_{\tilde{Q}}^{2}\right) Y_{d}^{\dagger} Y_{d} \operatorname{Tr}\left(Y_{e} Y_{e}^{\dagger}+3 Y_{d} Y_{d}^{\dagger}\right) \\
& \text { - }\left(4 m_{H_{u}}^{2}+m_{\tilde{Q}}^{2}\right) Y_{u}^{\dagger} Y_{u} \operatorname{Tr}\left(Y_{\nu} Y_{\nu}^{\dagger}+3 Y_{u} Y_{u}^{\dagger}\right) \\
& -2 Y_{d}^{\dagger} Y_{d} \operatorname{Tr}\left(Y_{e} m_{\tilde{L}}^{2} Y_{e}^{\dagger}+Y_{e}^{\dagger} m_{\tilde{e}}^{2} Y_{e}+3 Y_{d} m_{\tilde{Q}}^{2} Y_{d}^{\dagger}+3 Y_{d}^{\dagger} m_{\tilde{d}}^{2} Y_{d}\right) \\
& -2 Y_{u}^{\dagger} Y_{u} \operatorname{Tr}\left(Y_{\nu} m_{\tilde{L}}^{2} Y_{\nu}^{\dagger}+Y_{\nu}^{\dagger} m_{\tilde{\nu}}^{2} Y_{\nu}+3 Y_{u} m_{\tilde{Q}}^{2} Y_{u}^{\dagger}+3 Y_{u}^{\dagger} m_{\tilde{u}}^{2} Y_{u}\right) \\
& +\frac{2}{5} g_{1}^{2}\left(2 m_{H_{d}}^{2} Y_{d}^{\dagger} Y_{d}+4 m_{H_{u}}^{2} Y_{u}^{\dagger} Y_{u}+m_{\tilde{Q}}^{2} Y_{d}^{\dagger} Y_{d}+Y_{d}^{\dagger} Y_{d} m_{\tilde{Q}}^{2}+2 Y_{d}^{\dagger} m_{\tilde{d}}^{2} Y_{d}\right. \\
& \left.+2 m_{\tilde{Q}}^{2} Y_{u}^{\dagger} Y_{u}+2 Y_{u}^{\dagger} Y_{u} m_{\tilde{Q}}^{2}+4 Y_{u}^{\dagger} m_{\tilde{u}}^{2} Y_{u}+2 T_{d}^{\dagger} T_{d}+4 T_{u}^{\dagger} T_{u}\right) \\
& -\frac{4}{5} g_{1}^{2}\left(M_{1}^{*} Y_{d}^{\dagger} T_{d}+2 M_{1}^{*} Y_{u}^{\dagger} T_{u}-4\left|M_{1}\right|^{2} Y_{u}^{\dagger} Y_{u}\right. \\
& \left.+M_{1} T_{d}^{\dagger} Y_{d}+2 M_{1} T_{u}^{\dagger} Y_{u}-2\left|M_{1}\right|^{2} Y_{d}^{\dagger} Y_{d}\right) \\
& -\frac{128}{3} g_{3}^{4}\left|M_{3}\right|^{2} \mathbf{1}_{3}+\frac{2}{5} g_{1}^{2} g_{2}^{2} \operatorname{Re}\left(M_{1} M_{2}^{*}\right) \mathbf{1}_{3}+\frac{32}{45} g_{1}^{2} g_{3}^{2} \operatorname{Re}\left(M_{1} M_{3}^{*}\right) \mathbf{1}_{3} \\
& +\frac{199}{75} g_{1}^{4}\left|M_{1}\right|^{2} \mathbf{1}_{3}+\frac{2}{5} g_{1}^{2} g_{2}^{2}\left(\left|M_{1}\right|^{2}+\left|M_{2}\right|^{2}\right) \mathbf{1}_{3} \\
& +32 g_{2}^{2} g_{3}^{2}\left(\left|M_{2}\right|^{2}+\operatorname{Re}\left(M_{2} M_{3}^{*}\right)+\left|M_{3}\right|^{2}\right) \mathbf{1}_{3} \\
& +\frac{32}{45} g_{1}^{2} g_{3}^{2}\left(\left|M_{1}\right|^{2}+\left|M_{3}\right|^{2}\right) \mathbf{1}_{3}+33 g_{2}^{4}\left|M_{2}\right|^{2} \mathbf{1}_{3}
\end{aligned}
$$




$$
\begin{aligned}
& +\frac{1}{15} g_{1}^{2} \sigma_{1} \mathbf{1}_{3}+3 g_{2}^{2} \sigma_{2} \mathbf{1}_{3}+\frac{16}{3} g_{3}^{2} \sigma_{3} \mathbf{1}_{3}+\frac{2}{5} g_{1}^{2} S^{\prime} \mathbf{1}_{3}, \\
& \left(16 \pi^{2}\right)^{2} \beta_{m_{\tilde{u}}^{2}}^{(2)}=-2\left(2 m_{H_{d}}^{2}+2 m_{H_{u}}^{2}+m_{\tilde{u}}^{2}\right) Y_{u} Y_{d}^{\dagger} Y_{d} Y_{u}^{\dagger}-2\left(4 m_{H_{u}}^{2}+m_{\tilde{u}}^{2}\right) Y_{u} Y_{u}^{\dagger} Y_{u} Y_{u}^{\dagger} \\
& -4 T_{u}\left(T_{d}^{\dagger} Y_{d} Y_{u}^{\dagger}+T_{u}^{\dagger} Y_{u} Y_{u}^{\dagger}+Y_{d}^{\dagger} Y_{d} T_{u}^{\dagger}+Y_{u}^{\dagger} Y_{u} T_{u}^{\dagger}\right) \\
& -4 Y_{u}\left(T_{d}^{\dagger} T_{d} Y_{u}^{\dagger}+T_{u}^{\dagger} T_{u} Y_{u}^{\dagger}+Y_{d}^{\dagger} T_{d} T_{u}^{\dagger}+Y_{u}^{\dagger} T_{u} T_{u}^{\dagger}\right) \\
& -2 Y_{u}\left(2 Y_{d}^{\dagger} m_{\tilde{d}}^{2} Y_{d} Y_{u}^{\dagger}+Y_{d}^{\dagger} Y_{d} Y_{u}^{\dagger} m_{\tilde{u}}^{2}+2 Y_{d}^{\dagger} Y_{d} m_{\tilde{Q}}^{2} Y_{u}^{\dagger}+2 Y_{u}^{\dagger} m_{\tilde{u}}^{2} Y_{u} Y_{u}^{\dagger}\right. \\
& \left.+Y_{u}^{\dagger} Y_{u} Y_{u}^{\dagger} m_{\tilde{u}}^{2}+2 Y_{u}^{\dagger} Y_{u} m_{\tilde{Q}}^{2} Y_{u}^{\dagger}+2 m_{\tilde{Q}}^{2} Y_{d}^{\dagger} Y_{d} Y_{u}^{\dagger}+2 m_{\tilde{Q}}^{2} Y_{u}^{\dagger} Y_{u} Y_{u}^{\dagger}\right) \\
& -2\left(4 m_{H_{u}}^{2}+m_{\tilde{u}}^{2}\right) Y_{u} Y_{u}^{\dagger} \operatorname{Tr}\left(Y_{\nu} Y_{\nu}^{\dagger}+3 Y_{u} Y_{u}^{\dagger}\right) \\
& -4 T_{u}\left(T_{u}^{\dagger} \operatorname{Tr}\left(Y_{\nu} Y_{\nu}^{\dagger}+3 Y_{u} Y_{u}^{\dagger}\right)+Y_{u}^{\dagger} \operatorname{Tr}\left(Y_{\nu} T_{\nu}^{\dagger}+3 Y_{u} T_{u}^{\dagger}\right)\right) \\
& -4 Y_{u}\left(T_{u}^{\dagger} \operatorname{Tr}\left(T_{\nu} Y_{\nu}^{\dagger}+3 T_{u} Y_{u}^{\dagger}\right)+Y_{u}^{\dagger} \operatorname{Tr}\left(T_{\nu} T_{\nu}^{\dagger}+3 T_{u} T_{u}^{\dagger}\right)\right) \\
& -2 Y_{u}^{\dagger}\left(Y_{u}^{\dagger} m_{\tilde{u}}^{2} \operatorname{Tr}\left(Y_{\nu} Y_{\nu}^{\dagger}+3 Y_{u} Y_{u}^{\dagger}\right)+2 m_{\tilde{Q}}^{2} Y_{u}^{\dagger} \operatorname{Tr}\left(Y_{\nu} Y_{\nu}^{\dagger}+3 Y_{u} Y_{u}^{\dagger}\right)\right. \\
& \left.+2 Y_{u}^{\dagger} \operatorname{Tr}\left(Y_{\nu} m_{\tilde{L}}^{2} Y_{\nu}^{\dagger}+Y_{\nu}^{\dagger} m_{\tilde{\nu}}^{2} Y_{\nu}+3 Y_{u} m_{\tilde{Q}}^{2} Y_{u}^{\dagger}+3 Y_{u}^{\dagger} m_{\tilde{u}}^{2} Y_{u}\right)\right) \\
& +\left(6 g_{2}^{2}-\frac{2}{5} g_{1}^{2}\right)\left(m_{\tilde{u}}^{2} Y_{u} Y_{u}^{\dagger}+Y_{u} Y_{u}^{\dagger} m_{\tilde{u}}^{2}+2 m_{H_{u}}^{2} Y_{u} Y_{u}^{\dagger}+2 Y_{u} m_{\tilde{Q}}^{2} Y_{u}^{\dagger}+2 T_{u} T_{u}^{\dagger}\right) \\
& -12 g_{2}^{2}\left(M_{2}^{*} T_{u} Y_{u}^{\dagger}-2\left|M_{2}\right|^{2} Y_{u} Y_{u}^{\dagger}+M_{2} Y_{u} T_{u}^{\dagger}\right) \\
& +\frac{4}{5} g_{1}^{2}\left(M_{1}^{*} T_{u} Y_{u}^{\dagger}-2\left|M_{1}\right|^{2} Y_{u} Y_{u}^{\dagger}+M_{1} Y_{u} T_{u}^{\dagger}\right) \\
& -\frac{128}{3} g_{3}^{4}\left|M_{3}\right|^{2} \mathbf{1}_{3}+\frac{512}{45} g_{1}^{2} g_{3}^{2}\left(\left|M_{1}\right|^{2}+\operatorname{Re}\left(M_{1} M_{3}^{*}\right)+\left|M_{3}\right|^{2}\right) \mathbf{1}_{3} \\
& +\frac{3424}{75} g_{1}^{4}\left|M_{1}\right|^{2} \mathbf{1}_{3}+\frac{16}{15} g_{1}^{2} \sigma_{1} \mathbf{1}_{3}+\frac{16}{3} g_{3}^{2} \sigma_{3} \mathbf{1}_{3}-\frac{8}{5} g_{1}^{2} S^{\prime} \mathbf{1}_{3}, \\
& \left(16 \pi^{2}\right)^{2} \beta_{m_{\tilde{d}}^{2}}^{(2)}=-2\left(2 m_{H_{d}}^{2}+2 m_{H_{u}}^{2}+m_{\tilde{d}}^{2}\right) Y_{d} Y_{u}^{\dagger} Y_{u} Y_{d}^{\dagger}-2\left(4 m_{H_{d}}^{2}+m_{\tilde{d}}^{2}\right) Y_{d} Y_{d}^{\dagger} Y_{d} Y_{d}^{\dagger} \\
& -4 T_{d}\left(T_{d}^{\dagger} Y_{d} Y_{d}^{\dagger}+T_{u}^{\dagger} Y_{u} Y_{d}^{\dagger}+Y_{d}^{\dagger} Y_{d} T_{d}^{\dagger}+Y_{u}^{\dagger} Y_{u} T_{d}^{\dagger}\right) \\
& -4 Y_{d}\left(T_{d}^{\dagger} T_{d} Y_{d}^{\dagger}+T_{u}^{\dagger} T_{u} Y_{d}^{\dagger}+Y_{d}^{\dagger} T_{d} T_{d}^{\dagger}+Y_{u}^{\dagger} T_{u} T_{d}^{\dagger}\right) \\
& -2 Y_{d}\left(2 Y_{d}^{\dagger} m_{\tilde{d}}^{2} Y_{d} Y_{d}^{\dagger}+Y_{d}^{\dagger} Y_{d} Y_{d}^{\dagger} m_{\tilde{d}}^{2}+2 Y_{d}^{\dagger} Y_{d} m_{\tilde{Q}}^{2} Y_{d}^{\dagger}+2 Y_{u}^{\dagger} m_{\tilde{u}}^{2} Y_{u} Y_{d}^{\dagger}\right. \\
& \left.+Y_{u}^{\dagger} Y_{u} Y_{d}^{\dagger} m_{\tilde{d}}^{2}+2 Y_{u}^{\dagger} Y_{u} m_{\tilde{Q}}^{2} Y_{d}^{\dagger}+2 m_{\tilde{Q}}^{2} Y_{d}^{\dagger} Y_{d} Y_{d}^{\dagger}+2 m_{\tilde{Q}}^{2} Y_{u}^{\dagger} Y_{u} Y_{d}^{\dagger}\right) \\
& -2\left(4 m_{H_{d}}^{2}+m_{\tilde{d}}^{2}\right) Y_{d} Y_{d}^{\dagger} \operatorname{Tr}\left(Y_{e} Y_{e}^{\dagger}+3 Y_{d} Y_{d}^{\dagger}\right) \\
& -4 T_{d}\left(T_{d}^{\dagger} \operatorname{Tr}\left(Y_{e} Y_{e}^{\dagger}+3 Y_{d} Y_{d}^{\dagger}\right)+Y_{d}^{\dagger} \operatorname{Tr}\left(Y_{e} T_{e}^{\dagger}+3 Y_{d} T_{d}^{\dagger}\right)\right) \\
& -4 Y_{d}\left(T_{d}^{\dagger} \operatorname{Tr}\left(T_{e} Y_{e}^{\dagger}+3 T_{d} Y_{d}^{\dagger}\right)+Y_{d}^{\dagger} \operatorname{Tr}\left(T_{e} T_{e}^{\dagger}+3 T_{d} T_{d}^{\dagger}\right)\right) \\
& -2 Y_{d}^{\dagger}\left(Y_{d}^{\dagger} m_{\tilde{d}}^{2} \operatorname{Tr}\left(Y_{e} Y_{e}^{\dagger}+3 Y_{d} Y_{d}^{\dagger}\right)+2 m_{\tilde{Q}}^{2} Y_{d}^{\dagger} \operatorname{Tr}\left(Y_{e} Y_{e}^{\dagger}+3 Y_{d} Y_{d}^{\dagger}\right)\right. \\
& \left.+2 Y_{d}^{\dagger} \operatorname{Tr}\left(Y_{e} m_{\tilde{L}}^{2} Y_{e}^{\dagger}+Y_{e}^{\dagger} m_{\tilde{e}}^{2} Y_{e}+3 Y_{d} m_{\tilde{Q}}^{2} Y_{d}^{\dagger}+3 Y_{d}^{\dagger} m_{\tilde{d}}^{2} Y_{d}\right)\right) \\
& +\left(6 g_{2}^{2}+\frac{2}{5} g_{1}^{2}\right)\left(m_{\tilde{d}}^{2} Y_{d} Y_{d}^{\dagger}+Y_{d} Y_{d}^{\dagger} m_{\tilde{d}}^{2}+2 m_{H_{d}}^{2} Y_{d} Y_{d}^{\dagger}+2 Y_{d} m_{\tilde{Q}}^{2} Y_{d}^{\dagger}+2 T_{d} T_{d}^{\dagger}\right) \\
& -12 g_{2}^{2}\left(M_{2}^{*} T_{d} Y_{d}^{\dagger}-2\left|M_{2}\right|^{2} Y_{d} Y_{d}^{\dagger}+M_{2} Y_{d} T_{d}^{\dagger}\right)
\end{aligned}
$$




$$
\begin{aligned}
& -\frac{4}{5} g_{1}^{2}\left(M_{1}^{*} T_{d} Y_{d}^{\dagger}-2\left|M_{1}\right|^{2} Y_{d} Y_{d}^{\dagger}+M_{1} Y_{d} T_{d}^{\dagger}\right) \\
& -\frac{128}{3} g_{3}^{4}\left|M_{3}\right|^{2} \mathbf{1}_{3}+\frac{128}{45} g_{1}^{2} g_{3}^{2}\left(\left|M_{1}\right|^{2}+\operatorname{Re}\left(M_{1} M_{3}^{*}\right)+\left|M_{3}\right|^{2}\right) \mathbf{1}_{3} \\
& +\frac{808}{75} g_{1}^{4}\left|M_{1}\right|^{2} \mathbf{1}_{3}+\frac{4}{15} g_{1}^{2} \sigma_{1} \mathbf{1}_{3}+\frac{16}{3} g_{3}^{2} \sigma_{3} \mathbf{1}_{3}+\frac{4}{5} g_{1}^{2} S^{\prime} \mathbf{1}_{3}, \\
& \left(16 \pi^{2}\right)^{2} \beta_{m_{\tilde{e}}^{2}}^{(2)}=-2\left(2 m_{H_{d}}^{2}+2 m_{H_{u}}^{2}+m_{\tilde{e}}^{2}\right) Y_{e} Y_{\nu}^{\dagger} Y_{\nu} Y_{e}^{\dagger}-2\left(4 m_{H_{d}}^{2}+m_{\tilde{e}}^{2}\right) Y_{e} Y_{e}^{\dagger} Y_{e} Y_{e}^{\dagger} \\
& -4 T_{e}\left(T_{e}^{\dagger} Y_{e} Y_{e}^{\dagger}+T_{\nu}^{\dagger} Y_{\nu} Y_{e}^{\dagger}+Y_{e}^{\dagger} Y_{e} T_{e}^{\dagger}+Y_{\nu}^{\dagger} Y_{\nu} T_{e}^{\dagger}\right) \\
& -4 Y_{e}\left(T_{e}^{\dagger} T_{e} Y_{e}^{\dagger}+T_{\nu}^{\dagger} T_{\nu} Y_{e}^{\dagger}+Y_{e}^{\dagger} T_{e} T_{e}^{\dagger}+Y_{\nu}^{\dagger} T_{\nu} T_{e}^{\dagger}\right) \\
& -2 Y_{e}\left(2 Y_{e}^{\dagger} m_{\tilde{e}}^{2} Y_{e} Y_{e}^{\dagger}+Y_{e}^{\dagger} Y_{e} Y_{e}^{\dagger} m_{\tilde{e}}^{2}+2 Y_{e}^{\dagger} Y_{e} m_{\tilde{L}}^{2} Y_{e}^{\dagger}+2 Y_{\nu}^{\dagger} m_{\tilde{\nu}}^{2} Y_{\nu} Y_{e}^{\dagger}\right. \\
& \left.+Y_{\nu}^{\dagger} Y_{\nu} Y_{e}^{\dagger} m_{\tilde{e}}^{2}+2 Y_{\nu}^{\dagger} Y_{\nu} m_{\tilde{L}}^{2} Y_{e}^{\dagger}+2 m_{\tilde{L}}^{2} Y_{e}^{\dagger} Y_{e} Y_{e}^{\dagger}+2 m_{\tilde{L}}^{2} Y_{\nu}^{\dagger} Y_{\nu} Y_{e}^{\dagger}\right) \\
& -2\left(4 m_{H_{d}}^{2}+m_{\tilde{e}}^{2}\right) Y_{e} Y_{e}^{\dagger} \operatorname{Tr}\left(Y_{e} Y_{e}^{\dagger}+3 Y_{d} Y_{d}^{\dagger}\right) \\
& -4 T_{e}\left(T_{e}^{\dagger} \operatorname{Tr}\left(Y_{e} Y_{e}^{\dagger}+3 Y_{d} Y_{d}^{\dagger}\right)+Y_{e}^{\dagger} \operatorname{Tr}\left(Y_{e} T_{e}^{\dagger}+3 Y_{d} T_{d}^{\dagger}\right)\right) \\
& -4 Y_{e}\left(T_{e}^{\dagger} \operatorname{Tr}\left(T_{e} Y_{e}^{\dagger}+3 T_{d} Y_{d}^{\dagger}\right)+Y_{e}^{\dagger} \operatorname{Tr}\left(T_{e} T_{e}^{\dagger}+3 T_{d} T_{d}^{\dagger}\right)\right) \\
& -2 Y_{e}^{\dagger}\left(Y_{e}^{\dagger} m_{\tilde{e}}^{2} \operatorname{Tr}\left(Y_{e} Y_{e}^{\dagger}+3 Y_{d} Y_{d}^{\dagger}\right)+2 m_{\tilde{L}}^{2} Y_{e}^{\dagger} \operatorname{Tr}\left(Y_{e} Y_{e}^{\dagger}+3 Y_{d} Y_{d}^{\dagger}\right)\right. \\
& \left.+2 Y_{e}^{\dagger} \operatorname{Tr}\left(Y_{e} m_{\tilde{L}}^{2} Y_{e}^{\dagger}+Y_{e}^{\dagger} m_{\tilde{e}}^{2} Y_{e}+3 Y_{d} m_{\tilde{Q}}^{2} Y_{d}^{\dagger}+3 Y_{d}^{\dagger} m_{\tilde{d}}^{2} Y_{d}\right)\right) \\
& +\left(6 g_{2}^{2}-\frac{6}{5} g_{1}^{2}\right)\left(m_{\tilde{e}}^{2} Y_{e} Y_{e}^{\dagger}+Y_{e} Y_{e}^{\dagger} m_{\tilde{e}}^{2}+2 m_{H_{d}}^{2} Y_{e} Y_{e}^{\dagger}+2 Y_{e} m_{\tilde{L}}^{2} Y_{e}^{\dagger}+2 T_{e} T_{e}^{\dagger}\right) \\
& -12 g_{2}^{2}\left(M_{2}^{*} T_{e} Y_{e}^{\dagger}-2\left|M_{2}\right|^{2} Y_{e} Y_{e}^{\dagger}+M_{2} Y_{e} T_{e}^{\dagger}\right) \\
& +\frac{12}{5} g_{1}^{2}\left(M_{1}^{*} T_{e} Y_{e}^{\dagger}-2\left|M_{1}\right|^{2} Y_{e} Y_{e}^{\dagger}+M_{1} Y_{e} T_{e}^{\dagger}\right) \\
& +\frac{2808}{25} g_{1}^{4}\left|M_{1}\right|^{2} \mathbf{1}_{3}+\frac{12}{5} g_{1}^{2} \sigma_{1} \mathbf{1}_{3}+\frac{12}{5} g_{1}^{2} S^{\prime} \mathbf{1}_{3}, \\
& \left(16 \pi^{2}\right)^{2} \beta_{m_{\tilde{\nu}}^{2}}^{(2)}=-2\left(2 m_{H_{d}}^{2}+2 m_{H_{u}}^{2}+m_{\tilde{\nu}}^{2}\right) Y_{\nu} Y_{e}^{\dagger} Y_{e} Y_{\nu}^{\dagger}-2\left(4 m_{H_{u}}^{2}+m_{\tilde{\nu}}^{2}\right) Y_{\nu} Y_{\nu}^{\dagger} Y_{\nu} Y_{\nu}^{\dagger} \\
& -4 T_{\nu}\left(T_{e}^{\dagger} Y_{e} Y_{\nu}^{\dagger}+T_{\nu}^{\dagger} Y_{\nu} Y_{\nu}^{\dagger}+Y_{e}^{\dagger} Y_{e} T_{\nu}^{\dagger}+Y_{\nu}^{\dagger} Y_{\nu} T_{\nu}^{\dagger}\right) \\
& -4 Y_{\nu}\left(T_{e}^{\dagger} T_{e} Y_{\nu}^{\dagger}+T_{\nu}^{\dagger} T_{\nu} Y_{\nu}^{\dagger}+Y_{e}^{\dagger} T_{e} T_{\nu}^{\dagger}+Y_{\nu}^{\dagger} T_{\nu} T_{\nu}^{\dagger}\right) \\
& -2 Y_{\nu}\left(2 Y_{e}^{\dagger} m_{\tilde{e}}^{2} Y_{e} Y_{\nu}^{\dagger}+Y_{e}^{\dagger} Y_{e} Y_{\nu}^{\dagger} m_{\tilde{\nu}}^{2}+2 Y_{e}^{\dagger} Y_{e} m_{\tilde{L}}^{2} Y_{\nu}^{\dagger}+2 Y_{\nu}^{\dagger} m_{\tilde{\nu}}^{2} Y_{\nu} Y_{\nu}^{\dagger}\right. \\
& \left.+Y_{\nu}^{\dagger} Y_{\nu} Y_{\nu}^{\dagger} m_{\tilde{\nu}}^{2}+2 Y_{\nu}^{\dagger} Y_{\nu} m_{\tilde{L}}^{2} Y_{\nu}^{\dagger}+2 m_{\tilde{L}}^{2} Y_{e}^{\dagger} Y_{e} Y_{\nu}^{\dagger}+2 m_{\tilde{L}}^{2} Y_{\nu}^{\dagger} Y_{\nu} Y_{\nu}^{\dagger}\right) \\
& -2\left(4 m_{H_{u}}^{2}+m_{\tilde{\nu}}^{2}\right) Y_{\nu} Y_{\nu}^{\dagger} \operatorname{Tr}\left(Y_{\nu} Y_{\nu}^{\dagger}+3 Y_{u} Y_{u}^{\dagger}\right) \\
& -4 T_{\nu}\left(T_{\nu}^{\dagger} \operatorname{Tr}\left(Y_{\nu} Y_{\nu}^{\dagger}+3 Y_{u} Y_{u}^{\dagger}\right)+Y_{\nu}^{\dagger} \operatorname{Tr}\left(Y_{\nu} T_{\nu}^{\dagger}+3 Y_{u} T_{u}^{\dagger}\right)\right) \\
& -4 Y_{\nu}\left(T_{\nu}^{\dagger} \operatorname{Tr}\left(T_{\nu} Y_{\nu}^{\dagger}+3 T_{u} Y_{u}^{\dagger}\right)+Y_{\nu}^{\dagger} \operatorname{Tr}\left(T_{\nu} T_{\nu}^{\dagger}+3 T_{u} T_{u}^{\dagger}\right)\right) \\
& -2 Y_{\nu}^{\dagger}\left(Y_{\nu}^{\dagger} m_{\tilde{\nu}}^{2} \operatorname{Tr}\left(Y_{\nu} Y_{\nu}^{\dagger}+3 Y_{u} Y_{u}^{\dagger}\right)+2 m_{\tilde{L}}^{2} Y_{\nu}^{\dagger} \operatorname{Tr}\left(Y_{\nu} Y_{\nu}^{\dagger}+3 Y_{u} Y_{u}^{\dagger}\right)\right. \\
& \left.+2 Y_{\nu}^{\dagger} \operatorname{Tr}\left(Y_{\nu} m_{\tilde{L}}^{2} Y_{\nu}^{\dagger}+Y_{\nu}^{\dagger} m_{\tilde{\nu}}^{2} Y_{\nu}+3 Y_{u} m_{\tilde{Q}}^{2} Y_{u}^{\dagger}+3 Y_{u}^{\dagger} m_{\tilde{u}}^{2} Y_{u}\right)\right) \\
& +\left(6 g_{2}^{2}+\frac{6}{5} g_{1}^{2}\right)\left(m_{\tilde{\nu}}^{2} Y_{\nu} Y_{\nu}^{\dagger}+Y_{\nu} Y_{\nu}^{\dagger} m_{\tilde{\nu}}^{2}+2 m_{H_{u}}^{2} Y_{\nu} Y_{\nu}^{\dagger}+2 Y_{\nu} m_{\tilde{L}}^{2} Y_{\nu}^{\dagger}+2 T_{\nu} T_{\nu}^{\dagger}\right)
\end{aligned}
$$




$$
\begin{aligned}
& -12 g_{2}^{2}\left(M_{2}^{*} T_{\nu} Y_{\nu}^{\dagger}-2\left|M_{2}\right|^{2} Y_{\nu} Y_{\nu}^{\dagger}+M_{2} Y_{\nu} T_{\nu}^{\dagger}\right) \\
& -\frac{12}{5} g_{1}^{2}\left(M_{1}^{*} T_{\nu} Y_{\nu}^{\dagger}-2\left|M_{1}\right|^{2} Y_{\nu} Y_{\nu}^{\dagger}+M_{1} Y_{\nu} T_{\nu}^{\dagger}\right), \\
& \left(16 \pi^{2}\right)^{2} \beta_{m_{H_{d}}^{2}}^{(2)}=-2\left(m_{H_{d}}^{2}+m_{H_{u}}^{2}\right) \operatorname{Tr}\left(Y_{e} Y_{\nu}^{\dagger} Y_{\nu} Y_{e}^{\dagger}+3 Y_{d} Y_{u}^{\dagger} Y_{u} Y_{d}^{\dagger}\right) \\
& -12 m_{H_{d}}^{2} \operatorname{Tr}\left(Y_{e} Y_{e}^{\dagger} Y_{e} Y_{e}^{\dagger}+3 Y_{d} Y_{d}^{\dagger} Y_{d} Y_{d}^{\dagger}\right) \\
& -12 \operatorname{Tr}\left(T_{e}^{\dagger} T_{e} Y_{e}^{\dagger} Y_{e}+3 T_{d}^{\dagger} T_{d} Y_{d}^{\dagger} Y_{d}+T_{e}^{\dagger} Y_{e} Y_{e}^{\dagger} T_{e}+3 T_{d}^{\dagger} Y_{d} Y_{d}^{\dagger} T_{d}\right) \\
& -2 \operatorname{Tr}\left(T_{e} T_{\nu}^{\dagger} Y_{\nu} Y_{e}^{\dagger}+3 T_{d} T_{u}^{\dagger} Y_{u} Y_{d}^{\dagger}+T_{e} Y_{\nu}^{\dagger} Y_{\nu} T_{e}^{\dagger}+3 T_{d} Y_{u}^{\dagger} Y_{u} T_{d}^{\dagger}\right) \\
& -2 \operatorname{Tr}\left(Y_{e} T_{\nu}^{\dagger} T_{\nu} Y_{e}^{\dagger}+3 Y_{d} T_{u}^{\dagger} T_{u} Y_{d}^{\dagger}+Y_{e} Y_{\nu}^{\dagger} T_{\nu} T_{e}^{\dagger}+3 Y_{d} Y_{u}^{\dagger} T_{u} T_{d}^{\dagger}\right) \\
& -36 \operatorname{Tr}\left(Y_{d} Y_{d}^{\dagger} m_{\tilde{d}}^{2} Y_{d} Y_{d}^{\dagger}+Y_{d}^{\dagger} Y_{d} m_{\tilde{Q}}^{2} Y_{d}^{\dagger} Y_{d}\right) \\
& -12 \operatorname{Tr}\left(Y_{e} Y_{e}^{\dagger} m_{\tilde{e}}^{2} Y_{e} Y_{e}^{\dagger}+Y_{e}^{\dagger} Y_{e} m_{\tilde{L}}^{2} Y_{e}^{\dagger} Y_{e}\right) \\
& -6 \operatorname{Tr}\left(Y_{u} Y_{d}^{\dagger} m_{\tilde{d}}^{2} Y_{d} Y_{u}^{\dagger}+Y_{d} Y_{u}^{\dagger} m_{\tilde{u}}^{2} Y_{u} Y_{d}^{\dagger}+Y_{u}^{\dagger} Y_{u} m_{\tilde{Q}}^{2} Y_{d}^{\dagger} Y_{d}+Y_{d}^{\dagger} Y_{d} m_{\tilde{Q}}^{2} Y_{u}^{\dagger} Y_{u}\right) \\
& -2 \operatorname{Tr}\left(Y_{\nu} Y_{e}^{\dagger} m_{\tilde{e}}^{2} Y_{e} Y_{\nu}^{\dagger}+Y_{e} Y_{\nu}^{\dagger} m_{\tilde{\nu}}^{2} Y_{\nu} Y_{e}^{\dagger}+Y_{\nu}^{\dagger} Y_{\nu} m_{\tilde{L}}^{2} Y_{e}^{\dagger} Y_{e}+Y_{e}^{\dagger} Y_{e} m_{\tilde{L}}^{2} Y_{\nu}^{\dagger} Y_{\nu}\right) \\
& +\left(32 g_{3}^{2}-\frac{4}{5} g_{1}^{2}\right) \operatorname{Tr}\left(T_{d}^{\dagger} T_{d}+m_{H_{d}}^{2} Y_{d}^{\dagger} Y_{d}+Y_{d} m_{\tilde{Q}}^{2} Y_{d}^{\dagger}+Y_{d}^{\dagger} m_{\tilde{d}}^{2} Y_{d}\right) \\
& +\frac{12}{5} g_{1}^{2} \operatorname{Tr}\left(T_{e}^{\dagger} T_{e}+m_{H_{d}}^{2} Y_{e}^{\dagger} Y_{e}+Y_{e} m_{\tilde{L}}^{2} Y_{e}^{\dagger}+Y_{e}^{\dagger} m_{\tilde{e}}^{2} Y_{e}\right) \\
& -\frac{12}{5} g_{1}^{2} \operatorname{Tr}\left(M_{1}^{*} Y_{e}^{\dagger} T_{e}+M_{1} T_{e}^{\dagger} Y_{e}-2\left|M_{1}\right|^{2} Y_{e}^{\dagger} Y_{e}\right) \\
& +\frac{4}{5} g_{1}^{2} \operatorname{Tr}\left(M_{1}^{*} Y_{d}^{\dagger} T_{d}+M_{1} T_{d}^{\dagger} Y_{d}-2\left|M_{1}\right|^{2} Y_{d}^{\dagger} Y_{d}\right) \\
& -32 g_{3}^{2} \operatorname{Tr}\left(M_{3}^{*} Y_{d}^{\dagger} T_{d}+M_{3} T_{d}^{\dagger} Y_{d}-2\left|M_{3}\right|^{2} Y_{d}^{\dagger} Y_{d}\right) \\
& +\frac{18}{5} g_{1}^{2} g_{2}^{2}\left(\left|M_{1}\right|^{2}+\operatorname{Re}\left(M_{1} M_{2}^{*}\right)+\left|M_{2}\right|^{2}\right) \\
& +\frac{621}{25} g_{1}^{4}\left|M_{1}\right|^{2}+33 g_{2}^{4}\left|M_{2}\right|^{2}+\frac{3}{5} g_{1}^{2} \sigma_{1}+3 g_{2}^{2} \sigma_{2}-\frac{6}{5} g_{1}^{2} S^{\prime}, \\
& \left(16 \pi^{2}\right)^{2} \beta_{m_{H_{u}}^{2}}^{(2)}=-2\left(m_{H_{d}}^{2}+m_{H_{u}}^{2}\right) \operatorname{Tr}\left(Y_{e} Y_{\nu}^{\dagger} Y_{\nu} Y_{e}^{\dagger}+3 Y_{d} Y_{u}^{\dagger} Y_{u} Y_{d}^{\dagger}\right) \\
& -12 m_{H_{u}}^{2} \operatorname{Tr}\left(Y_{\nu} Y_{\nu}^{\dagger} Y_{\nu} Y_{\nu}^{\dagger}+3 Y_{u} Y_{u}^{\dagger} Y_{u} Y_{u}^{\dagger}\right) \\
& -12 \operatorname{Tr}\left(T_{\nu}^{\dagger} T_{\nu} Y_{\nu}^{\dagger} Y_{\nu}+3 T_{u}^{\dagger} T_{u} Y_{u}^{\dagger} Y_{u}+T_{\nu}^{\dagger} Y_{\nu} Y_{\nu}^{\dagger} T_{\nu}+3 T_{u}^{\dagger} Y_{u} Y_{u}^{\dagger} T_{u}\right) \\
& -2 \operatorname{Tr}\left(T_{e} T_{\nu}^{\dagger} Y_{\nu} Y_{e}^{\dagger}+3 T_{d} T_{u}^{\dagger} Y_{u} Y_{d}^{\dagger}+T_{e} Y_{\nu}^{\dagger} Y_{\nu} T_{e}^{\dagger}+3 T_{d} Y_{u}^{\dagger} Y_{u} T_{d}^{\dagger}\right) \\
& -2 \operatorname{Tr}\left(Y_{e} T_{\nu}^{\dagger} T_{\nu} Y_{e}^{\dagger}+3 Y_{d} T_{u}^{\dagger} T_{u} Y_{d}^{\dagger}+Y_{e} Y_{\nu}^{\dagger} T_{\nu} T_{e}^{\dagger}+3 Y_{d} Y_{u}^{\dagger} T_{u} T_{d}^{\dagger}\right) \\
& -36 \operatorname{Tr}\left(Y_{u} Y_{u}^{\dagger} m_{\tilde{u}}^{2} Y_{u} Y_{u}^{\dagger}+Y_{u}^{\dagger} Y_{u} m_{\tilde{Q}}^{2} Y_{u}^{\dagger} Y_{u}\right) \\
& -12 \operatorname{Tr}\left(Y_{\nu} Y_{\nu}^{\dagger} m_{\tilde{\nu}}^{2} Y_{\nu} Y_{\nu}^{\dagger}+Y_{\nu}^{\dagger} Y_{\nu} m_{\tilde{L}}^{2} Y_{\nu}^{\dagger} Y_{\nu}\right) \\
& -6 \operatorname{Tr}\left(Y_{u} Y_{d}^{\dagger} m_{\tilde{d}}^{2} Y_{d} Y_{u}^{\dagger}+Y_{d} Y_{u}^{\dagger} m_{\tilde{u}}^{2} Y_{u} Y_{d}^{\dagger}+Y_{u}^{\dagger} Y_{u} m_{\tilde{Q}}^{2} Y_{d}^{\dagger} Y_{d}+Y_{d}^{\dagger} Y_{d} m_{\tilde{Q}}^{2} Y_{u}^{\dagger} Y_{u}\right) \\
& -2 \operatorname{Tr}\left(Y_{\nu} Y_{e}^{\dagger} m_{\tilde{e}}^{2} Y_{e} Y_{\nu}^{\dagger}+Y_{e} Y_{\nu}^{\dagger} m_{\tilde{\nu}}^{2} Y_{\nu} Y_{e}^{\dagger}+Y_{\nu}^{\dagger} Y_{\nu} m_{\tilde{L}}^{2} Y_{e}^{\dagger} Y_{e}+Y_{e}^{\dagger} Y_{e} m_{\tilde{L}}^{2} Y_{\nu}^{\dagger} Y_{\nu}\right) \\
& +\left(32 g_{3}^{2}+\frac{8}{5} g_{1}^{2}\right) \operatorname{Tr}\left(T_{u}^{\dagger} T_{u}+m_{H_{u}}^{2} Y_{u}^{\dagger} Y_{u}+Y_{u} m_{\tilde{Q}}^{2} Y_{u}^{\dagger}+Y_{u}^{\dagger} m_{\tilde{u}}^{2} Y_{u}\right) \\
& -\frac{8}{5} g_{1}^{2} \operatorname{Tr}\left(M_{1}^{*} Y_{u}^{\dagger} T_{u}+M_{1} T_{u}^{\dagger} Y_{u}-2\left|M_{1}\right|^{2} Y_{u}^{\dagger} Y_{u}\right) \\
& -32 g_{3}^{2} \operatorname{Tr}\left(M_{3}^{*} Y_{u}^{\dagger} T_{u}+M_{3} T_{u}^{\dagger} Y_{u}-2\left|M_{3}\right|^{2} Y_{u}^{\dagger} Y_{u}\right)
\end{aligned}
$$




$$
\begin{aligned}
& +\frac{18}{5} g_{1}^{2} g_{2}^{2}\left(\left|M_{1}\right|^{2}+\operatorname{Re}\left(M_{1} M_{2}^{*}\right)+\left|M_{2}\right|^{2}\right) \\
& +\frac{621}{25} g_{1}^{4}\left|M_{1}\right|^{2}+33 g_{2}^{4}\left|M_{2}\right|^{2}+\frac{3}{5} g_{1}^{2} \sigma_{1}+3 g_{2}^{2} \sigma_{2}+\frac{6}{5} g_{1}^{2} S^{\prime},
\end{aligned}
$$

with

$$
\begin{aligned}
\sigma_{1} & =\frac{1}{5} g_{1}^{2}\left(3 m_{H_{u}}^{2}+3 m_{H_{d}}^{2}+\operatorname{Tr}\left(m_{\tilde{Q}}^{2}+3 m_{\tilde{L}}^{2}+2 m_{\tilde{d}}^{2}+6 m_{\tilde{e}}^{2}+8 m_{\tilde{u}}^{2}\right)\right) \\
\sigma_{2}^{2} & =g_{2}^{2}\left(m_{H_{u}}^{2}+m_{H_{d}}^{2}+\operatorname{Tr}\left(3 m_{\tilde{Q}}^{2}+m_{\tilde{L}}^{2}\right)\right) \\
\sigma_{3} & =g_{3}^{2} \operatorname{Tr}\left(2 m_{\tilde{Q}}^{2}+m_{\tilde{d}}^{2}+m_{\tilde{u}}^{2}\right)
\end{aligned}
$$

and

$$
\begin{aligned}
S^{\prime}= & m_{H_{d}}^{2} \operatorname{Tr}\left(Y_{e} Y_{e}^{\dagger}+3 Y_{d} Y_{d}^{\dagger}\right)-m_{H_{u}}^{2} \operatorname{Tr}\left(Y_{\nu} Y_{\nu}^{\dagger}+3 Y_{u} Y_{u}^{\dagger}\right) \\
& +\operatorname{Tr}\left(m_{\tilde{L}}^{2} Y_{e}^{\dagger} Y_{e}+m_{\tilde{L}}^{2} Y_{\nu}^{\dagger} Y_{\nu}\right)-\operatorname{Tr}\left(m_{\tilde{Q}}^{2} Y_{d}^{\dagger} Y_{d}+m_{\tilde{Q}}^{2} Y_{u}^{\dagger} Y_{u}\right) \\
& -2 \operatorname{Tr}\left(Y_{d} Y_{d}^{\dagger} m_{\tilde{d}}^{2}+Y_{e} Y_{e}^{\dagger} m_{\tilde{e}}^{2}-2 Y_{u} Y_{u}^{\dagger} m_{\tilde{u}}^{2}\right) \\
& +\frac{1}{30} g_{1}^{2}\left(9 m_{H_{u}}^{2}-9 m_{H_{d}}^{2}+\operatorname{Tr}\left(m_{\tilde{Q}}^{2}-9 m_{\tilde{L}}^{2}+4 m_{\tilde{d}}^{2}+36 m_{\tilde{e}}^{2}-32 m_{\tilde{u}}^{2}\right)\right) \\
& +\frac{3}{2} g_{2}^{2}\left(m_{H_{u}}^{2}-m_{H_{d}}^{2}+\operatorname{Tr}\left(m_{\tilde{Q}}^{2}-m_{\tilde{L}}^{2}\right)\right) \\
& +\frac{8}{3} g_{3}^{2} \operatorname{Tr}\left(m_{\tilde{Q}}^{2}+m_{\tilde{d}}^{2}-2 m_{\tilde{u}}^{2}\right) .
\end{aligned}
$$

\section{B Self-energies and one-loop tadpoles including inter-generational mix- ing}

Here we present the used formulas for the self-energies $\Pi_{Z Z}^{T}, \Pi_{H^{+} H^{-}}, \Pi_{A A}$, and the one-loop tadpoles $t_{u}, t_{d}$, which are based on [59] but generalized to include inter-generational mixing. In this appendix we employ SLHA 2 conventions [55] in the Super-CKM and Super-PMNS basis, to agree with the convention of [59]. The soft-breaking mass matrices in the SuperCKM/Super-PMNS basis are obtained from our flavour basis conventions (3.1) and (3.2) by

$$
\begin{aligned}
y_{f} & \equiv Y_{f}^{\text {diag }}=U_{f}^{\dagger} Y_{f} V_{f}, \\
\hat{T}_{f} & \equiv U_{f}^{\dagger} T_{f} V_{f}, \\
\hat{m}_{\tilde{Q}}^{2} & \equiv V_{d}^{\dagger} m_{\tilde{Q}}^{2} V_{d}, \\
\hat{m}_{\tilde{L}}^{2} & \equiv V_{e}^{\dagger} m_{\tilde{L}}^{2} V_{e}, \\
\hat{m}_{\tilde{u}}^{2} & \equiv U_{u}^{\dagger} m_{\tilde{u}}^{2} U_{u}, \\
\hat{m}_{\tilde{d}}^{2} & \equiv U_{d}^{\dagger} m_{\tilde{d}}^{2} U_{d}, \\
\hat{m}_{\tilde{e}}^{2} & \equiv U_{e}^{\dagger} m_{\tilde{e}}^{2} U_{e} .
\end{aligned}
$$

Let us briefly review our generalization to the sfermion mass matrices of [59]: we define the sfermion mixing matrices by ${ }^{10}$

$$
M_{\tilde{f}}^{2}=W_{\tilde{f}} M_{\tilde{f}}^{2 \operatorname{diag}} W_{\tilde{f}}^{\dagger},
$$

\footnotetext{
${ }^{10}$ The SLHA 2 convention sfermion mixing matrices $R_{\tilde{f}}$ can be obtained via $R_{\tilde{f}}=W_{\tilde{f}}^{\dagger}$.
} 
with the sfermion mass matrices in the Super-CKM/Super-PMNS basis

$$
\begin{aligned}
& M_{\tilde{u}}^{2}=\left(\begin{array}{cc}
V_{\mathrm{CKM}} \hat{m}_{\tilde{Q}}^{2} V_{\mathrm{CKM}}^{\dagger}+\frac{v^{2}}{2} y_{u}^{2} \sin ^{2} \beta+D_{u, L} & \frac{v}{\sqrt{2}} \hat{T}_{u}^{\dagger} \sin \beta-\mu \frac{v}{\sqrt{2}} y_{u} \cos \beta \\
\frac{v}{\sqrt{2}} \hat{T}_{u} \sin \beta-\mu^{*} \frac{v}{\sqrt{2}} y_{u} \cos \beta & \hat{m}_{\tilde{u}}^{2}+\frac{v^{2}}{2} y_{u}^{2} \sin ^{2} \beta+D_{u, R}
\end{array}\right), \\
& M_{\tilde{d}}^{2}=\left(\begin{array}{cc}
\hat{m}_{\tilde{Q}}^{2}+\frac{v^{2}}{2} y_{d}^{2} \cos ^{2} \beta+D_{d, L} & \frac{v}{\sqrt{2}} \hat{T}_{d}^{\dagger} \cos \beta-\mu \frac{v}{\sqrt{2}} y_{d} \sin \beta \\
\frac{v}{\sqrt{2}} \hat{T}_{d} \cos \beta-\mu^{*} \frac{v}{\sqrt{2}} y_{d} \sin \beta & \hat{m}_{\tilde{d}}^{2}+\frac{v^{2}}{2} y_{d}^{2} \cos ^{2} \beta+D_{d, R}
\end{array}\right), \\
& M_{\tilde{e}}^{2}=\left(\begin{array}{ll}
\hat{m}_{\tilde{L}}^{2}+\frac{v^{2}}{2} y_{e}^{2} \cos ^{2} \beta+D_{e, L} & \frac{v}{\sqrt{2}} \hat{T}_{e}^{\dagger} \cos \beta-\mu \frac{v}{\sqrt{2}} y_{e} \sin \beta \\
\frac{v}{\sqrt{2}} \hat{T}_{e} \cos \beta-\mu^{*} \frac{v}{\sqrt{2}} y_{e} \sin \beta & \hat{m}_{\tilde{e}}^{2}+\frac{v^{2}}{2} y_{e}^{2} \cos ^{2} \beta+D_{e, R}
\end{array}\right), \\
& M_{\tilde{\nu}}^{2}=V_{\mathrm{PMNS}}^{\dagger} \hat{m}_{\tilde{L}}^{2} V_{\mathrm{PMNS}}+D_{\nu, L} .
\end{aligned}
$$

The D-terms are given by

$$
\begin{aligned}
D_{f, L} & =\hat{M}_{Z}^{2}\left(I_{3}-Q_{e} \sin ^{2} \theta_{W}\right) \cos (2 \beta) \mathbf{1}_{3}, \\
D_{f, R} & =\hat{M}_{Z}^{2} Q_{e} \cos (2 \beta) \sin ^{2} \theta_{W} \mathbf{1}_{3},
\end{aligned}
$$

where $I_{3}$ denotes the $\mathrm{SU}(2)_{L}$ isospin and $Q_{e}$ the electric charge of the flavour $f$, and $\theta_{W}$ denotes the weak mixing angle. Note that our convention for $\mu$ differs by a sign from the convention in [59].

For the sake of completeness we also list the conventions for neutralino and chargino mass matrices and mixing matrices: the neutralino mixing matrix is defined by

$$
M_{\psi^{0}}=N^{T} M_{\psi^{0}}^{\operatorname{diag}} N
$$

with

$$
M_{\psi^{0}}=\left(\begin{array}{cccc}
M_{1} & 0 & -M_{Z} \cos \beta \sin \theta_{W} & M_{Z} \sin \beta \sin \theta_{W} \\
0 & M_{2} & M_{Z} \cos \beta \cos \theta_{W} & -M_{Z} \sin \beta \cos \theta_{W} \\
-M_{Z} \cos \beta \sin \theta_{W} & M_{Z} \cos \beta \cos \theta_{W} & 0 & -\mu \\
M_{Z} \sin \beta \sin \theta_{W} & -M_{Z} \sin \beta \cos \theta_{W} & -\mu & 0
\end{array}\right) .
$$

The chargino mixing matrix is defined by

$$
M_{\psi^{+}}=U^{T} M_{\psi^{+}}^{\text {diag }} V
$$

with

$$
M_{\psi^{+}}=\left(\begin{array}{cc}
M_{2} & \sqrt{2} M_{W} \sin \beta \\
\sqrt{2} M_{W} \cos \beta & \mu
\end{array}\right) .
$$

We now present the generalization of $\Pi_{Z Z}^{T}, \Pi_{H^{+} H^{-}}, \Pi_{A A}, t_{u}$, and $t_{d}$ of [59] to include inter-generational mixing. For all we have checked that our equations reduce to the corresponding equations in [59] when

$$
W_{\tilde{f}_{i i}}=W_{\tilde{f}_{i+3 i+3}}=\cos \theta_{\tilde{f}_{i}}
$$




$$
W_{\tilde{f}_{i i+3}}=-W_{\tilde{f}_{i+3 i}}=-\sin \theta_{\tilde{f}_{i}},
$$

where $i=1 \ldots 3$.

We keep the abbreviations of [59]:

$$
\begin{aligned}
s_{\alpha \beta} & \equiv \sin (\alpha-\beta), \\
c_{\alpha \beta} & \equiv \cos (\alpha-\beta), \\
g_{f_{L}} & \equiv I_{3}-Q_{e} \sin ^{2} \theta_{W}, \\
g_{f_{R}} & \equiv Q_{e} \sin ^{2} \theta_{W}, \\
e & \equiv g_{2} \sin \theta_{W}, \\
N_{C} & \equiv\left\{\begin{array}{ll}
3 & \text { for (s)quarks } \\
1 & \text { for (s)fermions }
\end{array} .\right.
\end{aligned}
$$

The conventions for the one-loop scalar functions $A_{0}, B_{22}, \tilde{B}_{22}, H, G$, and $F[77,78]$ are adopted from appendix B of [59]. Summations $\sum_{f}$ are over all fermions, whereas summations $\sum_{f_{u}}, \sum_{f_{d}}$ are restricted to up-type and down-type fermions, respectively. Summations $\sum_{Q}, \sum_{\tilde{Q}}$ are over $\mathrm{SU}(2)$ (s)quark doublets, and analogously for (s)leptons. In summations over sfermions the indices $i, j, s$, and $t$ run from 1 to 6 for $\tilde{u}, \tilde{d}$, and $\tilde{e}$ and from 1 to 3 for $\tilde{\nu}$. In summations of neutralinos (charginos) the indices $i, j$ run from 1 to 4 (2). The summations $\sum_{h^{0}}$ runs over all neutral Higgs- and Goldstone bosons, the summation $\sum_{h^{+}}$over the charged ones.

$$
\begin{aligned}
16 \pi^{2} \frac{\cos ^{2} \theta_{W}}{g_{2}^{2}} \Pi_{Z Z}^{T}\left(p^{2}\right)= & \left.-s_{\alpha \beta}^{2}\left(\tilde{B}_{22}\left(m_{A}, m_{H}\right)+\tilde{B}_{22}\left(M_{Z}, m_{h}\right)-M_{Z}^{2} B_{0}\left(M_{Z}, m_{h}\right)\right) \quad \text { B.1 }\right) \\
& -c_{\alpha \beta}^{2}\left(\tilde{B}_{22}\left(M_{Z}, m_{H}\right)+\tilde{B}_{22}\left(m_{A}, m_{h}\right)-M_{Z}^{2} B_{0}\left(M_{Z}, m_{h}\right)\right) \\
& -2 \cos ^{4} \theta_{W}\left(2 p^{2}+M_{W}^{2}-M_{Z}^{2} \frac{\sin ^{4} \theta_{W}}{\cos ^{2} \theta_{W}}\right) B_{0}\left(M_{W}, M_{W}\right) \\
& -\left(8 \cos ^{4} \theta_{W}+\cos ^{2}\left(2 \theta_{W}\right)\right) \tilde{B}_{22}\left(M_{W}, M_{W}\right) \\
& -\cos ^{2}\left(2 \theta_{W}\right) \tilde{B}_{22}\left(m_{H^{+}}, m_{H^{+}}\right) \\
& -\sum_{\tilde{f}} N_{C} \sum_{s, t}\left|I_{3} \sum_{i} W_{\tilde{f}_{i s}}^{*} W_{\tilde{f}_{i t}}-Q_{e} \sin ^{2} \theta_{W} \delta_{s t}\right|^{2} 4 B_{22}\left(m_{\tilde{f}_{s}}, m_{\tilde{f}_{t}}\right) \\
& +\frac{1}{2} \sum_{\tilde{f}} N_{C} \sum_{s}\left(\left(1-8 I_{3} Q_{e} \sin ^{2} \theta_{W}\right) \sum_{i} W_{\tilde{f}_{i s}}^{*} W_{\tilde{f}_{i s}}\right. \\
& \left.+4 Q_{e}^{2} \sin ^{4} \theta_{W}\right) A_{0}\left(m_{\tilde{f}_{s}}\right) \\
& +\sum_{f} N_{C}\left(\left(g_{f_{L}}^{2}+g_{f_{R}}^{2}\right) H\left(m_{f}, m_{f}\right)-4 g_{f_{L}} g_{f_{R}} m_{f}^{2} B_{0}\left(m_{f}, m_{f}\right)\right)
\end{aligned}
$$




$$
\begin{aligned}
& +\frac{\cos ^{2} \theta_{W}}{2 g_{2}^{2}} \sum_{i, j} f_{i j Z}^{0} H\left(m_{\tilde{\chi}_{i}^{0}}, m_{\tilde{\chi}_{j}^{0}}\right)+2 g_{i j Z}^{0} m_{\tilde{\chi}_{i}^{0}} m_{\tilde{\chi}_{j}^{0}} B_{0}\left(m_{\tilde{\chi}_{i}^{0}}, m_{\tilde{\chi}_{j}^{0}}\right) \\
& +\frac{\cos ^{2} \theta_{W}}{g_{2}^{2}} \sum_{i, j} f_{i j Z}^{+} H\left(m_{\tilde{\chi}_{i}^{+}}, m_{\tilde{\chi}_{j}^{+}}\right)+2 g_{i j Z}^{+} m_{\tilde{\chi}_{i}^{+}} m_{\tilde{\chi}_{j}^{+}} B_{0}\left(m_{\tilde{\chi}_{i}^{+}}, m_{\tilde{\chi}_{j}^{+}}\right) .
\end{aligned}
$$

The couplings $f_{Z}^{0}, f_{Z}^{+}, g_{Z}^{0}$, and $g_{Z}^{+}$are given in eqs. (A.7) and (D.5) of [59].

$$
\begin{aligned}
& 16 \pi^{2} \Pi_{H^{+} H^{-}}\left(p^{2}\right)=\sum_{Q} N_{C}\left(\left(\cos ^{2} \beta y_{u}^{2}+\sin ^{2} \beta y_{d}^{2}\right) G\left(m_{u}, m_{d}\right)\right. \\
& \left.-2 \sin (2 \beta) y_{u} y_{d} m_{u} m_{d} B_{0}\left(m_{u}, m_{d}\right)\right) \\
& +\sum_{L} \sin ^{2} \beta y_{e}^{2} G\left(0, m_{e}\right) \\
& +\sum_{\tilde{Q}} N_{C} \sum_{i, j}\left(\lambda_{H^{+}}\right)_{i j}^{2} B_{0}\left(m_{\tilde{u}_{i}}, m_{\tilde{d}_{j}}\right) \\
& +\sum_{\tilde{L}} \sum_{i, j}\left(\lambda_{H^{+} \tilde{L}}\right)_{i j}^{2} B_{0}\left(m_{\tilde{\nu}_{i}}, m_{\tilde{e}_{j}}\right) \\
& +\sum_{\tilde{f}} \sum_{i}\left(\lambda_{H^{+} H^{-} \tilde{f}}\right)_{i i} A_{0}\left(m_{\tilde{f}_{i}}\right) \\
& +\frac{g_{2}^{2}}{4}\left(s_{\alpha \beta}^{2} F\left(m_{H}, M_{W}\right)+c_{\alpha \beta}^{2} F\left(m_{h}, M_{W}\right)+F\left(m_{A}, M_{W}\right)\right. \\
& \left.+\frac{\cos ^{2}\left(2 \theta_{W}\right)}{\cos ^{2} \theta_{W}} F\left(m_{H^{+}}, M_{Z}\right)\right) \\
& +e^{2} F\left(m_{H^{+}}, 0\right)+2 g_{2}^{2} A_{0}\left(M_{W}\right)+g_{2}^{2} \cos ^{2}\left(2 \theta_{W}\right) A_{0}\left(M_{Z}\right) \\
& +\sum_{h^{+}}\left(\sum_{h^{0}}\left(\lambda_{H^{+} h^{0} h^{-}}\right)^{2} B_{0}\left(m_{h^{0}}, m_{h^{+}}\right)+\lambda_{H^{+} H^{-} h^{+} h^{-}} A_{0}\left(m_{h^{+}}\right)\right) \\
& +g_{2}^{2} \frac{M_{W}^{2}}{4} B_{0}\left(M_{W}, m_{A}\right) \\
& +\frac{1}{2} \sum_{h^{0}} \lambda_{H^{+} H^{-} h^{0} h^{0}} A_{0}\left(m_{h^{0}}\right) \\
& +\sum_{j, i} f_{i j H^{+}} G\left(m_{\tilde{\chi}_{j}^{+}}, m_{\tilde{\chi}_{i}^{0}}\right)-2 m_{\tilde{\chi}_{j}^{+}} m_{\tilde{\chi}_{i}^{0}} g_{i j H^{+}} B_{0}\left(m_{\tilde{\chi}_{j}^{+}}, m_{\tilde{\chi}_{i}^{0}}\right) \text {. }
\end{aligned}
$$

The couplings $f_{H^{+}}$and $g_{H^{+}}$are given in eq. (D.70) and eqs. (D.39)-(D.42) of [59]. The couplings $\lambda_{H^{+} H^{-} h^{-}}, \lambda_{H^{+} H^{-} h^{+} h^{-}}$, and $\lambda_{H^{+} H^{-} h^{0} h^{0}}$ are defined in eqs. (D.63)-(D.65) and eq. (D.67) of [59]. The couplings to sfermions in the case of inter-generational mixing are given by

$$
\begin{aligned}
& \lambda_{H^{+} \tilde{Q}}=W_{\tilde{u}}\left(\begin{array}{cc}
\frac{g 2}{\sqrt{2}} \hat{M}_{W} \sin (2 \beta) \mathbf{1}_{3}-\left(y_{u}^{2}+y_{d}^{2}\right) \cos \beta \frac{v \sin \beta}{\sqrt{2}} & -\hat{T}_{d}^{\dagger} \sin \beta-\mu y_{d} \cos \beta \\
-\hat{T}_{u} \cos \beta-\mu^{*} y_{u} \sin \beta & -y_{u} y_{d} \frac{v}{\sqrt{2}}
\end{array}\right) W_{\tilde{d}}, \\
& \lambda_{H+\tilde{L}}=W_{\tilde{\nu}}\left(\frac{g_{2}}{\sqrt{2}} \hat{M}_{W} \sin (2 \beta) \mathbf{1}_{3}-y_{e}^{2} \cos \beta \frac{v \sin \beta}{\sqrt{2}} \quad-\hat{T}_{e}^{\dagger} \sin \beta-\mu y_{e} \cos \beta\right) W_{\tilde{e}},
\end{aligned}
$$




$$
\begin{aligned}
& \lambda_{H^{+} H^{-} \tilde{f}}=-W_{\tilde{f}}^{\dagger}\left(\begin{array}{ccc}
-\frac{g_{2}^{2}}{2} \cos (2 \beta)\left(\frac{\cos \left(2 \theta_{W}\right)}{\cos ^{2} \theta_{W}} I_{3}+\tan ^{2} \theta_{W} Q_{e}\right) & \mathbf{1}_{3} & 0 \\
0 & \frac{g_{2}^{2}}{2} \cos (2 \beta) \tan ^{2} \theta_{W} Q_{e} \mathbf{1}_{3}
\end{array}\right) W_{\tilde{f}} \\
& +\left\{\begin{array}{l}
W_{\tilde{u}}^{\dagger}\left(\begin{array}{cc}
y_{d}^{2} \sin ^{2} \beta & 0 \\
0 & y_{u}^{2} \cos ^{2} \beta
\end{array}\right) W_{\tilde{u}} \quad \tilde{f}=\tilde{u} \\
W_{\tilde{d}}^{\dagger}\left(\begin{array}{cc}
y_{u}^{2} \cos ^{2} \beta & 0 \\
0 & y_{d}^{2} \sin ^{2} \beta
\end{array}\right) W_{\tilde{d}} \quad \tilde{f}=\tilde{d} \quad \\
W_{\tilde{e}}^{\dagger}\left(\begin{array}{cc}
0 & 0 \\
0 & y_{e}^{2} \sin ^{2} \beta
\end{array}\right) W_{\tilde{e}} \quad \tilde{f}=\tilde{e}
\end{array}\right. \\
& \lambda_{H^{+} H^{-} \tilde{\nu}}=-W_{\tilde{\nu}}^{\dagger}\left(-\frac{g_{2}^{2}}{2} \cos (2 \beta)\left(\frac{\cos \left(2 \theta_{W}\right)}{\cos ^{2} \theta_{W}} I_{3}+\tan ^{2} \theta_{W} Q_{e}\right) \mathbf{1}_{3}-y_{e}^{2} \sin ^{2} \beta\right) W_{\tilde{\nu}} \\
& 16 \pi^{2} \Pi_{A A}\left(p^{2}\right)=\cos ^{2} \beta \sum_{f_{u}} N_{C} y_{f_{u}}^{2}\left(p^{2} B_{0}\left(m_{f_{u}}, m_{f_{u}}\right)-2 A_{0}\left(m_{f_{u}}\right)\right) \\
& +\sin ^{2} \beta \sum_{f_{d}} N_{C} y_{f_{d}}^{2}\left(p^{2} B_{0}\left(m_{f_{d}}, m_{f_{d}}\right)-2 A_{0}\left(m_{f_{d}}\right)\right) \\
& +\sum_{\tilde{f}} \sum_{i} N_{C}\left(\left(\lambda_{A A \tilde{f}}\right)_{i i} A_{0}\left(m_{\tilde{f}_{i}}\right)+\sum_{j}\left(\lambda_{A} \tilde{f}\right)_{i j}^{2} B_{0}\left(m_{\tilde{f}_{i}}, m_{\tilde{f}_{j}}\right)\right) \\
& +\frac{g_{2}^{2}}{4}\left(2 F\left(m_{H^{+}}, M_{W}\right)+\frac{s_{\alpha \beta}^{2}}{\cos ^{2} \theta_{W}} F\left(m_{H}, M_{Z}\right)+\frac{c_{\alpha \beta}^{2}}{\cos ^{2} \theta_{W}} F\left(m_{h}, M_{Z}\right)\right) \\
& +\frac{1}{2} \sum_{h_{n}^{0}}\left(\sum_{h_{m}^{0}} \lambda_{A h_{n}^{0} h_{m}^{0}} B_{0}\left(m_{h_{n}^{0}}, m_{h_{m}^{0}}\right)+\lambda_{A A h_{n}^{0} h_{n}^{0}} A_{0}\left(m_{h_{n}^{0}}\right)\right) \\
& +g_{2}^{2}\left(\frac{M_{W}^{2}}{2} B_{0}\left(M_{W}, m_{H_{p}}\right)+2 A_{0}\left(M_{W}\right)+\frac{1}{\cos ^{2} \theta_{W}} A_{0}\left(M_{Z}\right)\right) \\
& +\sum_{h^{+}} \lambda_{A A h^{+} h^{+}} A_{0}\left(m_{h^{+}}\right) \\
& +\frac{1}{2} \sum_{i, j} f_{i j A}^{0} G\left(m_{\tilde{\chi}_{i}^{0}}, m_{\tilde{\chi}_{j}^{0}}\right)-2 g_{i j A}^{0} m_{\tilde{\chi}_{i}^{0}} m_{\tilde{\chi}_{j}^{0}} B_{0}\left(m_{\tilde{\chi}_{i}^{0}}, m_{\tilde{\chi}_{j}^{0}}\right) \\
& +\sum_{i, j} f_{i j A}^{+} G\left(m_{\tilde{\chi}_{i}^{+}}, m_{\tilde{\chi}_{j}^{+}}\right)-2 g_{i j A}^{+} m_{\tilde{\chi}_{i}^{+}} m_{\tilde{\chi}_{j}^{+}} B_{0}\left(m_{\tilde{\chi}_{i}^{+}}, m_{\tilde{\chi}_{j}^{+}}\right) .
\end{aligned}
$$

The couplings $f_{A}^{0}, g_{A}^{0}, f_{A}^{+}$, and $g_{A}^{+}$are given in eq. (D.70) and eqs. (D.34-D.38) of [59]. The couplings $\lambda_{A h^{0} h^{0}}, \lambda_{A A h^{0} h^{0}}$, and $\lambda_{A A h^{+} h^{+}}$are defined in eqs. (D.63-D.65) and eq. (D.67) of [59]. The couplings to sfermions in the case of inter-generational mixing are given by

$$
\begin{aligned}
\lambda_{A \tilde{u}} & =W_{\tilde{u}}^{\dagger}\left(\begin{array}{cc}
0 & -\frac{1}{\sqrt{2}}\left(\hat{T}_{u}^{\dagger} \cos \beta+\mu y_{u} \sin \beta\right)
\end{array}\right) W_{\tilde{u}}, \\
\lambda_{A \tilde{e}(\tilde{d})} & =W_{\tilde{e}(\tilde{d})}^{\dagger}\left(\begin{array}{c}
\left.\hat{T}_{u} \cos \beta+\mu^{*} y_{u} \sin \beta\right) \\
0
\end{array}\right. \\
\lambda_{A \tilde{\nu}} & =0
\end{aligned}
$$




$$
\begin{aligned}
& \lambda_{A A \tilde{f}}=W_{\tilde{f}}^{\dagger}\left(\begin{array}{cc}
-\frac{g_{2}^{2}}{2} \cos (2 \beta)\left(\frac{1}{\cos ^{2} \theta_{W}} I_{3}-\tan ^{2} \theta_{W} Q_{e}\right) \mathbf{1}_{3} & 0 \\
0 & -\frac{g_{2}^{2}}{2} \cos (2 \beta) \tan ^{2} \theta_{W} Q_{e} \mathbf{1}_{3}
\end{array}\right) W_{\tilde{f}} \\
& +\left\{\begin{array}{cc}
W_{\tilde{u}}^{\dagger}\left(\begin{array}{cc}
y_{u}^{2} \cos ^{2} \beta & 0 \\
0 & y_{u}^{2} \cos ^{2} \beta
\end{array}\right) W_{\tilde{u}} & \tilde{f}=\tilde{u} \\
W_{\tilde{d}}^{\dagger}\left(\begin{array}{cc}
y_{d}^{2} \sin ^{2} \beta & 0 \\
0 & y_{d}^{2} \sin ^{2} \beta
\end{array}\right) W_{\tilde{d}} & \tilde{f}=\tilde{d} \quad, \\
W_{\tilde{e}}^{\dagger}\left(\begin{array}{cc}
y_{e}^{2} \sin ^{2} \beta & 0 \\
0 & y_{e}^{2} \sin ^{2} \beta
\end{array}\right) W_{\tilde{e}} & \tilde{f}=\tilde{e}
\end{array}\right. \\
& \lambda_{A A \tilde{\nu}}=-\frac{g_{2}^{2}}{2} \cos (2 \beta)\left(\frac{1}{\cos ^{2} \theta_{W}} I_{3}-\tan ^{2} \theta_{W} Q_{e}\right) \mathbf{1}_{3} \\
& 16 \pi^{2} t_{d}=-2 \sum_{f_{d}} N_{C} y_{f_{d}}^{2} A_{0}\left(m_{f_{d}}\right) \\
& +\sum_{\tilde{f}} N_{C} \sum_{i} \frac{g_{2}^{2}}{2 M_{W} \cos \beta}\left(\lambda_{d \tilde{f}}\right)_{i i} A_{0}\left(m_{\tilde{f}_{i}}\right) \\
& -g_{2}^{2} \frac{\cos (2 \beta)}{8 \cos ^{2} \theta_{W}}\left(A_{0}\left(m_{A}\right)+2 A_{0}\left(m_{H^{+}}\right)\right)+\frac{g_{2}^{2}}{2} A_{0}\left(m_{H_{p}}\right) \\
& +\frac{g_{2}^{2}}{8 \cos ^{2} \theta_{W}}\left(3 \sin ^{2} \alpha-\cos ^{2} \alpha+\sin (2 \alpha) \tan \beta\right) A_{0}\left(m_{h}\right) \\
& +\frac{g_{2}^{2}}{8 \cos ^{2} \theta_{W}}\left(3 \cos ^{2} \alpha-\sin ^{2} \alpha-\sin (2 \alpha) \tan \beta\right) A_{0}\left(m_{H}\right) \\
& -g_{2}^{2} \sum_{i} \frac{m_{\tilde{\chi}_{i}^{0}}}{M_{W} \cos \beta} \operatorname{Re}\left(N_{i 3}\left(N_{i 2}-N_{i, 1} \tan \theta_{W}\right)\right) A_{0}\left(m_{\tilde{\chi}_{i}^{0}}\right) \\
& -\sqrt{2} g_{2}^{2} \sum_{i} \frac{m_{\tilde{\chi}_{i}^{+}}}{M_{W} \cos \beta} \operatorname{Re}\left(V_{i 1} U_{i 2}\right) A_{0}\left(m_{\tilde{\chi}_{i}^{+}}\right) \\
& +\frac{3}{4} g_{2}^{2}\left(2 A_{0}\left(M_{W}\right)+\frac{A_{0}\left(M_{Z}\right)}{\cos ^{2} \theta_{W}}\right) \\
& +g_{2}^{2} \frac{\cos (2 \beta)}{8 \cos ^{2} \theta_{W}}\left(2 A_{0}\left(M_{W}\right)+A_{0}\left(M_{Z}\right)\right) \text {. }
\end{aligned}
$$

The couplings to sfermion in the case of inter-generational mixing are given by

$$
\begin{aligned}
& \lambda_{d \tilde{u}}=W_{\tilde{u}}^{\dagger}\left(\begin{array}{ccc}
g_{2} \frac{M_{Z}}{\cos \theta_{W}} g_{u_{L}} \cos \beta & \mathbf{1}_{3} & -y_{u} \frac{\mu}{\sqrt{2}} \\
-y_{u} \frac{\mu^{*}}{\sqrt{2}} & & g_{2} \frac{M_{Z}}{\cos \theta_{W}} g_{u_{R}} \cos \beta \mathbf{1}_{3}
\end{array}\right) W_{\tilde{u}}, \\
& \lambda_{d \tilde{f}}=W_{\tilde{f}}^{\dagger}\left(\begin{array}{ccc}
g_{2} \frac{M_{Z}}{\cos \theta_{W}} g_{f_{L}} \cos \beta & \mathbf{1}_{3} & \hat{T}_{f}^{\dagger} \frac{1}{\sqrt{2}} \\
\hat{T}_{f} \frac{1}{\sqrt{2}} & g_{2} \frac{M_{Z}}{\cos \theta_{W}} g_{f_{R}} \cos \beta \mathbf{1}_{3}
\end{array}\right) \\
& +\left\{\begin{array}{cc}
W_{\tilde{d}}^{\dagger}\left(\begin{array}{cc}
Y_{d}^{2} v \cos \beta & 0 \\
0 & Y_{d}^{2} v \cos \beta
\end{array}\right) W_{\tilde{d}} & \tilde{f}=\tilde{d} \\
W_{\tilde{e}}^{\dagger}\left(\begin{array}{cc}
Y_{e}^{2} v \cos \beta & 0 \\
0 & Y_{e}^{2} v \cos \beta
\end{array}\right) W_{\tilde{e}} & \tilde{f}=\tilde{e}
\end{array},\right.
\end{aligned}
$$




$$
\begin{aligned}
\lambda_{d \tilde{\nu}}= & g_{2} \frac{M_{Z}}{\cos \theta_{W}} g_{\nu_{L}} \cos \beta \mathbf{1}_{3} \\
16 \pi^{2} t_{u}= & -2 \sum_{f_{u}} N_{C} y_{f_{u}}^{2} A_{0}\left(m_{f_{u}}\right) \\
& +\sum_{\tilde{f}} N_{C} \sum_{i} \frac{g_{2}^{2}}{2 M_{W} \sin \beta}\left(\lambda_{u \tilde{f}}\right)_{i i} A_{0}\left(m_{\tilde{f}_{i}}\right) \\
& +g_{2}^{2} \frac{\cos (2 \beta)}{8 \cos ^{2} \theta_{W}}\left(A_{0}\left(m_{A}\right)+2 A_{0}\left(m_{H^{+}}\right)\right)+\frac{g_{2}^{2}}{2} A_{0}\left(m_{H_{p}}\right) \\
& +\frac{g_{2}^{2}}{8 \cos ^{2} \theta_{W}}\left(3 \cos ^{2} \alpha-\sin ^{2} \alpha+\sin (2 \alpha) \cot \beta\right) A_{0}\left(m_{h}\right) \\
& +\frac{g_{2}^{2}}{8 \cos ^{2} \theta_{W}}\left(3 \sin ^{2} \alpha-\cos ^{2} \alpha-\sin (2 \alpha) \cot \beta\right) A_{0}\left(m_{H}\right) \\
& -g_{2}^{2} \sum_{i} \frac{m_{\tilde{\chi}_{i}^{0}}}{M_{W} \sin \beta} R e\left(N_{i 4}\left(N_{i 2}-N_{i, 1} \tan \theta_{W}\right)\right) A_{0}\left(m_{\tilde{\chi}_{i}^{0}}\right) \\
& -\sqrt{2} g_{2}^{2} \sum_{i} \frac{m_{\tilde{\chi}_{i}^{+}}}{M_{W} \sin \beta} R e\left(V_{i 2} U_{i 1}\right) A_{0}\left(m_{\tilde{\chi}_{i}^{+}}\right) \\
& +\frac{3}{4} g_{2}^{2}\left(2 A_{0}\left(M_{W}\right)+\frac{A_{0}\left(M_{Z}\right)}{\cos ^{2} \theta_{W}}\right) \\
& -g_{2}^{2} \frac{\cos (2 \beta)}{8 \cos ^{2} \theta_{W}}\left(2 A_{0}\left(M_{W}\right)+A_{0}\left(M_{Z}\right)\right) .
\end{aligned}
$$

The couplings to sfermion in the case of inter-generational mixing are given by

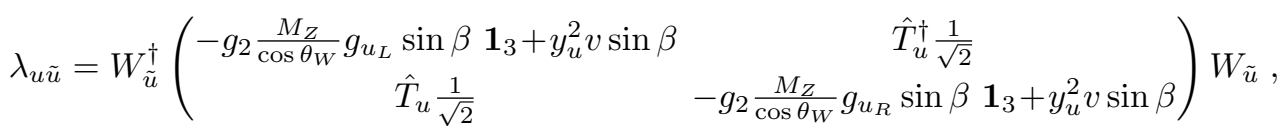

$$
\begin{aligned}
& \lambda_{u \tilde{f}}=W_{\tilde{f}}^{\dagger}\left(\begin{array}{ccc}
-g_{2} \frac{M_{Z}}{\cos \theta_{W}} g_{f_{L}} \sin \beta \mathbf{1}_{3} & -y_{\tilde{f}} \frac{\mu}{\sqrt{2}} \\
-y_{\tilde{f}} \frac{\mu^{*}}{\sqrt{2}} & -g_{2} \frac{M_{Z}}{\cos \theta_{W}} g_{f_{R}} \sin \beta \mathbf{1}_{3}
\end{array}\right) \quad \tilde{f}=\tilde{e}, \tilde{d}, \\
& \lambda_{u \tilde{\nu}}=-g_{2} \frac{M_{Z}}{\cos \theta_{W}} g_{\nu_{L}} \sin \beta \mathbf{1}_{3} \text {. }
\end{aligned}
$$

\section{SusyTC documentation}

Here we present a documentation of the REAP extension SusyTC. To get started, please follow first the steps described in section 4 .

We now describe that additional features of SusyTC: in addition to the features of REAP package RGEMSSMsoftbroken.m (described in the REAP documentation), SusyTC adds the following options to the command RGEAdd:

- $\operatorname{STCsign} \mu$ is the general factor $e^{i \phi_{\mu}}$ in front of $\mu$ in (3.36). (default: +1$)$

- STCcMSSM is a switch to change between the CP-violating (complex) MSSM and CPconserving (real) MSSM. (default: True) 
- STCSusyscale sets the SUSY scale $Q$ (in GeV), where the MSSM is matched to the SM. If set to "Automatic", SusyTC determines $Q$ automatically from the sparticle spectrum. (default: "Automatic")

- STCSusyScaleFromStops is a switch to choose whether SusyTC calculates the SUSY scale $Q$ as geometric mean of the stop masses $Q=\sqrt{m_{\tilde{t}_{1}} m_{\tilde{t}_{2}}}$, where the stop masses are defined by the up-type squark mass eigenstates $\tilde{u}_{i}$ with the largest mixing to $\tilde{t}_{1}$ and $\tilde{t}_{2}$, or as geometric mean of the lightest and heaviest up-type squarks $Q=\sqrt{m_{\tilde{u}_{1}} m_{\tilde{u}_{6}}}$. Without effect if STCSusyScale is not set to "Automatic". (default: True)

- STCSearchSMTransition is a switch to enable or disable the matching to the SM and the calculation of supersymmetric threshold corrections and sparticle spectrum. (default: True)

- STCCCBConstraints is a switch to enable or disable a warning message for potentially dangerous charge and colour breaking vacua, if large trilinear couplings violate the constraints of [79] at the SUSY scale $Q$

$$
\left|T_{i j}\right|^{2} \leq\left(m_{R_{i i}}^{2}+m_{L_{j j}}^{2}+m_{H_{f}}^{2}+|\mu|^{2}\right)\left\{\begin{array}{l}
\left(y_{i}^{2}+y_{j}^{2}\right) \quad i \neq j \\
3 y_{i}^{2} \quad i=j
\end{array},\right.
$$

where $m_{L}, m_{R}$ and $m_{H_{f}}$ denote the soft-breaking mass parameters of the scalar fields associated with the trilinear coupling $T$ in the basis of diagonal Yukawa matrices. (default: True)

- STCUFBConstraints is a switch to enable or disable a warning message for possibly dangerous "unbounded from below" directions in the scalar potential, if the constraints of [69] are violated at the SUSY scale $Q$

$$
\begin{aligned}
m_{H_{u}}^{2}+|\mu|^{2}+m_{\tilde{L}_{i}}^{2}-\frac{\left|m_{3}\right|^{4}}{m_{H_{d}}^{2}+|\mu|^{2}-m_{\tilde{L}_{i}}^{2}}>0 & \text { UFB-2 } \\
m_{H_{u}}^{2}+m_{\tilde{L}_{i}}^{2}>0 & \text { UFB-3 },
\end{aligned}
$$

evaluated in the basis of (3.11). Note that the UFB-I constraint is automatically satisfied, since SusyTC calculates $m_{3}^{2}$ from $m_{H_{u}}^{2}, m_{H_{d}}^{2}, M_{Z}$, and $\tan \beta$ by requiring the existence of electroweak symmetry breaking. (default: True)

- STCTachyonConstraints is a switch to enable or disable the rejection of a parameter point with tachyonic running masses of sfermions at any renormalization scale above the SUSY scale $Q$. Regardless of this switch, tachyonic sfermion masses at $Q$ are always rejected. (default: False)

Thus, a typical call of RGEAdd [] might look like

RGEAdd["MSSMsoftbroken" , RGEtan $\beta->20$, STCcMSSM->False , STCsign $\mu->-1$ ]; 
In addition to the parameters known from the MSSM REAP model, the following softbreaking parameters are available as input for RGESetInitial:

- RGETu, RGETd, RGETe, and RGET $\nu$ are the soft-breaking trilinear coupling matrices. If given, the Constrained MSSM parameter RGEAO for the corresponding trilinear coupling is overwritten. (default: Constrained MSSM)

- RGEM1, RGEM2, and RGEM3 are the soft-breaking gaugino mass parameters. If given, the Constrained MSSM parameter RGEM12 for the corresponding gaugino is overwritten. (default: Constrained MSSM)

- RGEm2Q, RGEm2L, RGEm2u, RGEm2d, RGEm2e, RGEm2 $\nu$ are the soft-breaking squared mass matrices $m_{\tilde{f}}^{2}$ for the sfermions. If given, the Constrained MSSM parameter RGEm0 for the corresponding scalar masses is overwritten. (default: Constrained MSSM)

- RGEm2Hd and RGEm2Hu are the soft-breaking squared masses for $H_{d}$ and $H_{u}$, respectively. If given, the Constrained MSSM parameter RGEm0 for the corresponding scalar mass is overwritten. (default: Constrained MSSM)

- RGEM12 is the Constrained MSSM parameter for gaugino mass parameters in GeV. (default: 750 )

- RGEm0 is the Constrained MSSM parameter for all soft-breaking masses of scalars in GeV. (default: 1500)

- RGEA0 is the Constrained MSSM parameter $A_{0}$ for trilinear couplings, e.g. $T_{f}=A_{0} Y_{f}$. (default: -500)

An example for an input at the GUT scale would be

$$
\begin{aligned}
& \text { RGESetInitial[2·10^16, RGEM1->863, RGEM2->131, } \\
& \text { RGEM3->-392, RGESuggestion->"GUT"]; }
\end{aligned}
$$

The solution at a lower energy scale such as $M_{Z}$ can now be obtained by the REAP command RGESolve:

RGESolve[91.19,2.10^16];

Some parameter points might lead to tachyonic sparticle masses. In such instances the evaluation of SusyTC is stopped and an error message is returned using the Mathematica command Throw. In order to properly catch such error messages, we therefore recommend to use instead

Catch[RGESolve[91.19,2·10^16], TachyonicMass];

In addition to the parameters known from the MSSM REAP model, the following softbreaking parameters are available for RGEGetSolution at all energy scales higher than the SUSY scale $Q$ : 
- RGETu, RGETd, RGETe, and RGET $\nu$ are used to get the soft-breaking trilinear coupling matrices.

- $\operatorname{RawT} \nu$ is used to get the raw (internal representation) of the soft-breaking trilinear matrix for sneutrinos.

- RGEM1, RGEM2, and RGEM3 are used to get the soft-breaking gaugino mass parameters.

- RGEm2Q, RGEm2L, RGEm2u, RGEm2d, RGEm2e, RGEm2 $\nu$ are used to get the soft-breaking squared mass matrices $m_{\tilde{f}}^{2}$ for the sfermions.

- RGEm2Hd and RGEm2Hu are used to get the soft-breaking squared masses for $H_{d}$ and $H_{u}$, respectively.

To obtain the running $\overline{\mathrm{DR}}$ gluino mass at a scale of two TeV for example, one uses

RGEGetSolution[2000, RGEM3];

With SusyTC the $\overline{\mathrm{DR}}$ sparticle spectrum is automatically calculated. The following functions are included in SusyTC:

- STCGetSUSYScale [] returns the SUSY scale $Q$.

- STCGetSUSYSpectrum[] returns a list of replacement rules for the SUSY scale $Q$, the $\overline{\mathrm{DR}}$ tree-level values of $\mu$ and $m_{3}$, and the $\overline{\mathrm{DR}}$ sparticle masses and (tree-level) mixing matrices at the SUSY scale. In detail it contains

- "Q" the SUSY scale $Q$.

- " $\mu$ ", " $m_{3}$ " the values of $\mu$ and $m_{3}$.

- "M1", "M2" , "M3" are the gaugino mass parameters.

- "Mh", "MH" , "MA", "MHp" the (tree-level) masses of the MSSM Higgs bosons. ${ }^{11}$

- "m $\chi 0 "$ a list of the four neutralino masses.

- "m $\chi \mathrm{p} "$ a list of the two chargino masses.

- "msude" a $3 \times 6$ array of the six up-type quarks, down-type squarks and charged slepton masses, respectively.

- "ms $\nu$ " a list of the three light sneutrino masses.

- $" \theta \mathrm{W} "$ the weak mixing angle.

- " $\tan \alpha "$ the mixing angle of the CP-even Higgs bosons.

- "N" the mixing matrix of neutralinos.

- "U", "V" the mixing matrices for charginos.

- "Wude" a list of the three sparticle mixing matrices for up-type squarks, downtype squarks and charged sleptons.

\footnotetext{
${ }^{11}$ Note that there is no CP-violation in the MSSM Higgs sector on tree-level.
} 
- "W $\nu$ " the mixing matrix of the three light sneutrinos.

To obtain for example the SUSY scale and the tree-level masses of the charginos call

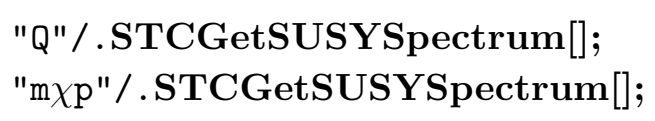

The squark masses and charged slepton masses are contained in a joint list as $\left\{m_{\tilde{u}}, m_{\tilde{d}}, m_{\tilde{e}}\right\}$, and analogously for the sfermion mixing matrices. To obtain for example the up-type squark masses, the charged slepton mixing matrix, and the sneutrino masses type

\section{("msude" /.STCGetSUSYSpectrum[]) [[1]]; \\ ("Wude" /.STCGetSUSYSpectrum[]) [[3] ] ; \\ "ms $\nu "$. .STCGetSUSYSpectrum[];}

- STCGetOneLoopValues [] returns a list of replacement rules containing

- " $\mu$ " , " $m_{3}$ " the one-loop corrected $\overline{\mathrm{DR}} \mu$-parameter and $m_{3}$ as in (3.36) and (3.37) at the SUSY scale $Q$.

- "vev" the one-loop $\overline{\mathrm{DR}}$ vev $\hat{v}$ as in (3.41) at the SUSY scale $Q$.

- "MHp" ("MA") the pole-mass $m_{H^{+}}\left(m_{A}\right)$ of the charged (CP-odd) Higgs boson for STCCMSSM $=$ True (False).

The value of $\mu$ can for example be obtained from

\section{$" \mu " /$.STCGetOneLoopValues[];}

- STCGetSCKMValues [] returns a list of replacement rules with the soft-breaking mass squared and trilinear coupling matrices in the SCKM basis, where sparticles are rotated analogously with their corresponding superpartners. ${ }^{12}$ Since they are used for the self-energies calculation as described in the previous appendix, they are returned in SLHA2 convention [55]! In detail, there are

- "VCKM" the CKM mixing matrix.

- "VPMNS" the PMNS mixing matrix.

- SCKMBasis ["m2Q"], SCKMBasis["m2u"], SCKMBasis["m2d"] the squark softbreaking mass squared matrices in the super CKM basis with SLHA2 convetions.

- SCKMBasis["m2L"], SCKMBasis["m2e"] the slepton soft-breaking mass squared matrices in the super PMNS basis with SLHA2 conventions.

- SCKMBasis["T"] a list of the three trilinear coupling matrices for up-type squarks, down-type squarks and charged sleptons in the SCKM basis with SLHA2 conventions.

\footnotetext{
${ }^{12}$ We use the term "SCKM" for the super CKM and super PMNS basis.
} 
- SCKMBasis ["Y"] a $3 \times 3$ array of the Yukawa coupling singular values for up-type squarks, down-type squarks and charged sleptons.

To obtain the down-type trilinear coupling matrix and the mass squared matrix of the left-handed up-type squarks in the SLHA basis for example, type

\section{(SCKMBasis ["T"]/.STCGetSCKMValues[]) [[2]] ; SCKMBasis ["mQ2u"]/.STCGetSCKMValues[];}

- STCGetInternalValues [] returns everything that is internally used for the calculation of the threshold corrections and sparticle spectrum, i.e. the results from STCGetSCKMValues [] and STCGetSUSYSpectrum [] with the one-loop corrected parameters from STCGetOneLoopValues [] replacing tree-level ones if available. We recommend to the user to use those separate functions instead.

As additional feature, SusyTC optionally supports input and output as SLHA "Les Houches" files. These files follow SLHA conventions [55, 58]:

- STCSLHA2Input [ "Path"] loads an "Les Houches" input file stored in "Path" and executes REAP and SusyTC. If no path is given, the default path is assumed as "SusyTC.in" in the Mathematica Notebook Directory. An important difference to other spectrum calculators is the pure "top-down" approach by SusyTC, i.e. there is no attempt of fitting SM inputs at a low scale or calculating a GUT-scale from gauge couplings unification. Instead, all input is given at a user-defined high energy scale, which is then evolved to lower scales. The input should be given in the flavour basis in SLHA 2 convention [55], with analogous convention for $Y_{\nu}$ and convention for $M_{n}$ as in (3.1). The relation between the SusyTC conventions and the SLHA 2 conventions is given in section 3. In the following, we list all SLHA 2 input blocks, which are available in SusyTC:

- Block MODSEL: the only available switch is:

$5:($ Default $=2)$ CP violation $($ STCcMSSM $)$

- Block SusyTCInput: Switches (1=True, 0=False) are defined for RGEAdd []:

1 : (Default $=1)$ STCSusyScaleFromStops

$2:($ Default $=1)$ STCSearchSMTransition

$3:($ Default $=1)$ STCCCBConstraints

$4:($ Default $=1)$ STCUFBConstraints

5 : (Default $=1)$ Print a Warning in case of Tachyonic masses

$6:($ Default $=1)$ One or Two Loop RGEs

- Block MINPAR: Constrained MSSM parameters as defined in [55, 58]. Note however, that the input value of $\tan \beta$ is interpreted to be given at the SUSY scale.

- Block IMMINPAR: reads the $\sin \phi_{\mu}$ in case of the complex MSSM:

$4: \sin \phi_{\mu}$ 
- Block EXTPAR:

$0 \quad:\left(\right.$ Default $\left.=2 \cdot 10^{16}\right): M_{\text {input }}$ Input scale

Note that with SusyTC an automatic calculation of the GUT scale is not possible.

The remainder of the block works as usual, e.g. optionally one can overwrite common Constrained MSSM gaugino or Higgs soft-breaking parameters:

$1: M_{1}\left(M_{\text {input }}\right)$ bino mass (real part)

$2: M_{2}\left(M_{\text {input }}\right)$ wino mass (real part)

$3: M_{3}\left(M_{\text {input }}\right)$ gluino mass (real part)

$21: m_{H_{d}}^{2}\left(M_{\text {input }}\right)$

$22: m_{H_{u}}^{2}\left(M_{\text {input }}\right)$

Imaginary components for the gaugino masses can be given in Block IMEXTPAR.

- Block IMEXTPAR: as defined in [55].

- Block QEXTPAR: low energy input:

0 : (Default $=91.1876)$ : the low energy scale to which REAP evolves the SM RGEs.

23 : "SUSY scale" $Q$, where the MSSM is matched to the SM. If this entry is set, it overwrites the automatically calculated SUSY scale.

- Block GAUGE: the $\overline{\mathrm{DR}}$ gauge couplings at the input scale

$1: g_{1}\left(M_{\text {input }}\right) \mathrm{U}(1)$ gauge coupling

$2: g_{2}\left(M_{\text {input }}\right) \mathrm{SU}(2)$ gauge coupling

$3: g_{3}\left(M_{\text {input }}\right) \mathrm{SU}(3)$ gauge coupling

- Block YU, Block YD, Block YE, Block YN: the real parts of the Yukawa matrices $Y_{u}, Y_{d}, Y_{e}$, and $Y_{\nu}$ in the flavour basis [55]. They should be given in the FORTRAN format ( $1 \mathrm{x}, \mathrm{I} 2,1 \mathrm{x}, \mathrm{I} 2,3 \mathrm{x}, 1 \mathrm{P}, \mathrm{E} 16.8, \mathrm{OP}, 3 \mathrm{x}$, ' \#' , $1 \mathrm{x}, \mathrm{A})$, where the first two integers correspond to the indices and the double precision number to $\operatorname{Re}\left(Y_{i j}\right)$.

- Block IMYU, Block IMYD, Block IMYE, Block IMYN: the imaginary parts of the Yukawa matrices $Y_{u}, Y_{d}, Y_{e}$, and $Y_{\nu}$ in the flavour basis [55]. They are given in the same format as the real parts.

- Block MN: the real part of the symmetric Majorana mass matrix $M_{n}$ of the righthanded neutrinos in the flavour basis (3.1). Only the "upper-triangle" entries should be given, the input format is as for the Yukawa matrices.

- Block IMMN: the imaginary part of the symmetric Majorana mass matrix $M_{n}$ of the right-handed neutrinos in the flavour basis (3.1). Only the "upper-triangle" entries should be given, the input format is as for the Yukawa matrices.

The remaining blocks can be given optionally to overwrite Constrained MSSM input boundary conditions:

- Block TU, Block TD, Block TE, Block TN: the real parts of the trilinear soft-breaking matrices $T_{u}, T_{d}, T_{e}$, and $T_{\nu}$ in the flavour basis [55]. They should be given in the same format as the Yukawa matrices. 
- Block IMTU, Block IMTD, Block IMTE, Block IMTN: the imaginary parts of the trilinear soft-breaking matrices $T_{u}, T_{d}, T_{e}$, and $T_{\nu}$ in the flavour basis [55]. They should be given in the same format as the Yukawa matrices.

- Block MSQ2, Block MSU2, Block MSD2, Block MSL2, Block MSE2, Block MSN2: the real parts of the soft-breaking mass squared matrices $m_{\tilde{Q}}^{2}, m_{\tilde{u}}^{2}$, $m_{\tilde{d}}^{2}, m_{\tilde{L}}^{2}, m_{\tilde{e}}^{2}$, and $m_{\tilde{\nu}}^{2}$ in the flavour basis [55]. Only the "upper-triangle" entries should be given, the input format is as for the Yukawa matrices.

- Block IMMSQ2, Block IMMSU2, Block IMMSD2,Block IMMSL2, Block IMMSE2, Block IMMSN2: the imaginary parts of the soft-breaking mass squared matrices $m_{\tilde{Q}}^{2}, m_{\tilde{u}}^{2}, m_{\tilde{d}}^{2}, m_{\tilde{L}}^{2}, m_{\tilde{e}}^{2}$, and $m_{\tilde{\nu}}^{2}$ in the flavour basis [55]. Only the "upper-triangle" entries should be given, the input format is as for the Yukawa matrices.

- STCWriteSLHA2Output ["Path"] writes an "Les Houches" [55, 58] output file to "Path". If no path is given, the output is saved in the Mathematica Notebook directory as "SusyTC.out". The output follows SLHA conventions, with the following exceptions:

- Block MASS: the mass spectrum is given as $\overline{\mathrm{DR}}$ masses at the SUSY scale. The only exception is the pole mass $M_{H^{+}}\left(M_{A}\right)$ for CP violation turned on (off).

- Block ALPHA: the tree-level Higgs mixing angle $\alpha_{\text {tree }}$.

- Block HMIX: instead of $M_{A}$ we give $101: m_{3}$

The other blocks follow the SLHA2 output conventions, e.g. $\overline{\mathrm{DR}}$ values at the SUSY scale in the Super-CKM/Super-PMNS basis. To avoid confusion, the blocks Block DSQMIX, Block USQMIX, Block SELMIX, Block SNUMIX and the corresponding blocks for the imaginary entries, return the sfermion mixing matrices $R_{\tilde{f}}$ in SLHA 2 convention.

Open Access. This article is distributed under the terms of the Creative Commons Attribution License (CC-BY 4.0), which permits any use, distribution and reproduction in any medium, provided the original author(s) and source are credited.

\section{References}

[1] S. Antusch, J. Kersten, M. Lindner, M. Ratz and M.A. Schmidt, Running neutrino mass parameters in see-saw scenarios, JHEP 03 (2005) 024 [hep-ph/0501272] [INSPIRE].

[2] R. Hempfling, Yukawa coupling unification with supersymmetric threshold corrections, Phys. Rev. D 49 (1994) 6168 [INSPIRE].

[3] L.J. Hall, R. Rattazzi and U. Sarid, The top quark mass in supersymmetric $\mathrm{SO}(10)$ unification, Phys. Rev. D 50 (1994) 7048 [hep-ph/9306309] [INSPIRE]. 
[4] M. Carena, M. Olechowski, S. Pokorski and C.E.M. Wagner, Electroweak symmetry breaking and bottom-top Yukawa unification, Nucl. Phys. B 426 (1994) 269 [hep-ph/9402253] [INSPIRE].

[5] T. Blazek, S. Raby and S. Pokorski, Finite supersymmetric threshold corrections to CKM matrix elements in the large $\tan \beta$ regime, Phys. Rev. D 52 (1995) 4151 [hep-ph/9504364] [INSPIRE].

[6] S. Antusch and M. Spinrath, Quark and lepton masses at the GUT scale including SUSY threshold corrections, Phys. Rev. D 78 (2008) 075020 [arXiv:0804.0717] [INSPIRE].

[7] L.E. Ibáñez and G.G. Ross, Low-energy predictions in supersymmetric Grand Unified Theories, Phys. Lett. B 105 (1981) 439 [INSPIRE].

[8] M.B. Einhorn and D.R.T. Jones, The weak mixing angle and unification mass in supersymmetric SU(5), Nucl. Phys. B 196 (1982) 475 [INSPIRE].

[9] J.R. Ellis, D.V. Nanopoulos and S. Rudaz, GUTs 3: SUSY GUTs 2, Nucl. Phys. B 202 (1982) 43 [INSPIRE].

[10] S. Dimopoulos and H. Georgi, Softly broken supersymmetry and SU(5), Nucl. Phys. B 193 (1981) 150 [INSPIRE].

[11] H. Georgi and C. Jarlskog, A new lepton-quark mass relation in a unified theory, Phys. Lett. B 86 (1979) 297 [INSPIRE].

[12] S. Antusch and M. Spinrath, New GUT predictions for quark and lepton mass ratios confronted with phenomenology, Phys. Rev. D 79 (2009) 095004 [arXiv:0902.4644] [INSPIRE].

[13] S. Antusch, S.F. King and M. Spinrath, GUT predictions for quark-lepton Yukawa coupling ratios with messenger masses from non-singlets, Phys. Rev. D 89 (2014) 055027 [arXiv: 1311.0877] [INSPIRE].

[14] G. Ross and M. Serna, Unification and fermion mass structure, Phys. Lett. B 664 (2008) 97 [arXiv: 0704.1248] [INSPIRE].

[15] W. Altmannshofer, D. Guadagnoli, S. Raby and D.M. Straub, SUSY GUTs with Yukawa unification: a go/no-go study using FCNC processes, Phys. Lett. B 668 (2008) 385 [arXiv: 0801.4363] [INSPIRE].

[16] I. Gogoladze, R. Khalid, S. Raza and Q. Shafi, $t-b-\tau$ Yukawa unification for $\mu<0$ with a sub-TeV sparticle spectrum, JHEP 12 (2010) 055 [arXiv:1008.2765] [INSPIRE].

[17] S. Antusch, L. Calibbi, V. Maurer and M. Spinrath, From flavour to SUSY flavour models, Nucl. Phys. B 852 (2011) 108 [arXiv:1104.3040] [INSPIRE].

[18] H. Baer, I. Gogoladze, A. Mustafayev, S. Raza and Q. Shafi, Sparticle mass spectra from SU(5) SUSY GUT models with b- $\tau$ Yukawa coupling unification, JHEP 03 (2012) 047 [arXiv: 1201.4412] [INSPIRE].

[19] H. Baer, S. Raza and Q. Shafi, A heavier gluino from t-b- $\tau$ Yukawa-unified SUSY, Phys. Lett. B 712 (2012) 250 [arXiv:1201.5668] [INSPIRE].

[20] A. Anandakrishnan, S. Raby and A. Wingerter, Yukawa unification predictions for the LHC, Phys. Rev. D 87 (2013) 055005 [arXiv: 1212.0542] [inSPIRE].

[21] M. Adeel Ajaib, I. Gogoladze, Q. Shafi and C.S. Un, A predictive Yukawa unified SO(10) model: Higgs and sparticle masses, JHEP 07 (2013) 139 [arXiv:1303.6964] [INSPIRE]. 
[22] N. Okada, S. Raza and Q. Shafi, Particle spectroscopy of supersymmetric SU(5) in light of 125 GeV Higgs and muon g-2 data, Phys. Rev. D 90 (2014) 015020 [arXiv:1307.0461] [INSPIRE].

[23] A. Anandakrishnan, B.C. Bryant, S. Raby and A. Wingerter, LHC phenomenology of $\mathrm{SO}(10)$ models with Yukawa unification, Phys. Rev. D 88 (2013) 075002 [arXiv:1307.7723] [INSPIRE].

[24] B. Bajc, S. Lavignac and T. Mede, Challenging the minimal supersymmetric SU(5) model, AIP Conf. Proc. 1604 (2014) 297 [arXiv: 1310.3093] [INSPIRE].

[25] M.A. Ajaib, I. Gogoladze, Q. Shafi and C.S. Ün, Split sfermion families, Yukawa unification and muon g-2, JHEP 05 (2014) 079 [arXiv: 1402.4918] [INSPIRE].

[26] A. Anandakrishnan, B.C. Bryant and S. Raby, LHC phenomenology of $\mathrm{SO}(10)$ models with Yukawa unification II, Phys. Rev. D 90 (2014) 015030 [arXiv:1404.5628] [INSPIRE].

[27] A. Anandakrishnan, B.C. Bryant and S. Raby, Threshold corrections to the bottom quark mass revisited, JHEP 05 (2015) 088 [arXiv:1411.7035] [INSPIRE].

[28] I. Gogoladze, A. Mustafayev, Q. Shafi and C.S. Un, Yukawa unification and sparticle spectroscopy in gauge mediation models, Phys. Rev. D 91 (2015) 096005 [arXiv:1501.07290] [INSPIRE].

[29] Z. Poh and S. Raby, Yukawa unification in an $\mathrm{SO}(10)$ SUSY GUT: SUSY on the edge, Phys. Rev. D 92 (2015) 015017 [arXiv: 1505.00264] [INSPIRE].

[30] Z. Berezhiani, M. Chianese, G. Miele and S. Morisi, Chances for SUSY-GUT in the LHC epoch, JHEP 08 (2015) 083 [arXiv: 1505.04950] [INSPIRE].

[31] B. Bajc, S. Lavignac and T. Mede, Resurrecting the minimal renormalizable supersymmetric SU(5) model, JHEP 01 (2016) 044 [arXiv: 1509. 06680] [INSPIRE].

[32] ATLAS collaboration, Observation of a new particle in the search for the Standard Model Higgs boson with the ATLAS detector at the LHC, Phys. Lett. B 716 (2012) 1 [arXiv: 1207.7214] [INSPIRE].

[33] CMS collaboration, Observation of a new boson at a mass of $125 \mathrm{GeV}$ with the CMS experiment at the LHC, Phys. Lett. B 716 (2012) 30 [arXiv:1207.7235] [INSPIRE].

[34] S. Antusch, C. Gross, V. Maurer and C. Sluka, A flavour GUT model with $\theta_{13}^{\text {PMNS }} \simeq \theta_{C} / \sqrt{2}$, Nucl. Phys. B 877 (2013) 772 [arXiv:1305.6612] [INSPIRE].

[35] S. Antusch, C. Gross, V. Maurer and C. Sluka, Inverse neutrino mass hierarchy in a flavour GUT model, Nucl. Phys. B 879 (2014) 19 [arXiv:1306.3984] [INSPIRE].

[36] S. Antusch and V. Maurer, Running quark and lepton parameters at various scales, JHEP 11 (2013) 115 [arXiv: 1306.6879] [INSPIRE].

[37] H. Baer, F.E. Paige, S.D. Protopopescu and X. Tata, Simulating supersymmetry with ISAJET 7.0/ISASUSY 1.0, hep-ph/9305342 [INSPIRE].

[38] B.C. Allanach, SOFTSUSY: a program for calculating supersymmetric spectra, Comput. Phys. Commun. 143 (2002) 305 [hep-ph/0104145] [INSPIRE].

[39] A. Djouadi, J.-L. Kneur and G. Moultaka, SuSpect: a fortran code for the supersymmetric and Higgs particle spectrum in the MSSM, Comput. Phys. Commun. 176 (2007) 426 [hep-ph/0211331] [INSPIRE]. 
[40] W. Porod, SPheno, a program for calculating supersymmetric spectra, SUSY particle decays and SUSY particle production at $e^{+} e^{-}$colliders, Comput. Phys. Commun. 153 (2003) 275 [hep-ph/0301101] [INSPIRE].

[41] W. Porod and F. Staub, SPheno 3.1: extensions including flavour, CP-phases and models beyond the MSSM, Comput. Phys. Commun. 183 (2012) 2458 [arXiv:1104.1573] [INSPIRE].

[42] A. Djouadi, SUSY calculation tools, hep-ph/0211357 [INSPIRE].

[43] S. Antusch, I. de Medeiros Varzielas, V. Maurer, C. Sluka and M. Spinrath, Towards predictive flavour models in SUSY SU(5) GUTs with doublet-triplet splitting, JHEP 09 (2014) 141 [arXiv:1405.6962] [INSPIRE].

[44] J. Gehrlein, J.P. Oppermann, D. Schäfer and M. Spinrath, An SU(5) $\times A_{5}$ golden ratio flavour model, Nucl. Phys. B 890 (2014) 539 [arXiv:1410.2057] [InSPIRE].

[45] A. Meroni, S.T. Petcov and M. Spinrath, A SUSY SU(5) $\times T^{\prime}$ unified model of flavour with large $\theta_{13}$, Phys. Rev. D 86 (2012) 113003 [arXiv:1205.5241] [INSPIRE].

[46] S. Antusch, C. Gross, V. Maurer and C. Sluka, $\theta_{13}^{\text {PMNS }}=\theta_{C} / \sqrt{2}$ from GUTs, Nucl. Phys. B 866 (2013) 255 [arXiv:1205.1051] [INSPIRE].

[47] S. Antusch and M. Ratz, Supergraph techniques and two loop $\beta$-functions for renormalizable and nonrenormalizable operators, JHEP 07 (2002) 059 [hep-ph/0203027] [INSPIRE].

[48] P. Minkowski, $\mu \rightarrow$ er at a rate of one out of $10^{9}$ muon decays?, Phys. Lett. B 67 (1977) 421 [inSPIRE].

[49] P. Ramond, The family group in Grand Unified Theories, in Sanibel Talk, CALT-68-709, February 1979 [hep-ph/9809459] [INSPIRE].

[50] M. Gell-Mann, P. Ramond and R. Slansky, Complex spinors and unified theories, in Supergravity, North Holland, Amsterdam The Netherlands (1979) [Conf. Proc. C 790927 (1979) 315] [arXiv: 1306.4669] [INSPIRE].

[51] T. Yanagida, Horizontal symmetry and masses of neutrinos, in Proc. of the Workshop on Unified Theory and Baryon Number of the Universe, KEK Japan (1979) [INSPIRE].

[52] S.L. Glashow, The future of elementary particle physics, in Cargese Lectures, Cargese France (1979) [NATO Sci. Ser. B 61 (1980) 687] [InSPIRE].

[53] R.N. Mohapatra and G. Senjanović, Neutrino mass and spontaneous parity violation, Phys. Rev. Lett. 44 (1980) 912 [INSPIRE].

[54] J. Schechter and J.W.F. Valle, Neutrino decay and spontaneous violation of lepton number, Phys. Rev. D 25 (1982) 774 [inSPIRE].

[55] B.C. Allanach et al., SUSY Les Houches accord 2, Comput. Phys. Commun. 180 (2009) 8 [arXiv: 0801.0045] [INSPIRE].

[56] S.P. Martin and M.T. Vaughn, Two loop renormalization group equations for soft supersymmetry breaking couplings, Phys. Rev. D 50 (1994) 2282 [Erratum ibid. D 78 (2008) 039903] [hep-ph/9311340] [INSPIRE].

[57] S. Antusch and E. Cazzato, One-loop right-handed neutrino threshold corrections for two-loop running in supersymmetric type I seesaw models, JHEP 12 (2015) 066 [arXiv: 1509.05604] [INSPIRE].

[58] P.Z. Skands et al., SUSY Les Houches accord: interfacing SUSY spectrum calculators, decay packages and event generators, JHEP 07 (2004) 036 [hep-ph/0311123] [INSPIRE]. 
[59] D.M. Pierce, J.A. Bagger, K.T. Matchev and R.-J. Zhang, Precision corrections in the minimal supersymmetric standard model, Nucl. Phys. B 491 (1997) 3 [hep-ph/9606211] [INSPIRE].

[60] S. Heinemeyer, W. Hollik and G. Weiglein, FeynHiggs: a program for the calculation of the masses of the neutral CP even Higgs bosons in the MSSM, Comput. Phys. Commun. 124 (2000) 76 [hep-ph/9812320] [INSPIRE].

[61] S. Heinemeyer, W. Hollik and G. Weiglein, The masses of the neutral CP-even Higgs bosons in the MSSM: accurate analysis at the two loop level, Eur. Phys. J. C 9 (1999) 343 [hep-ph/9812472] [INSPIRE].

[62] G. Degrassi, S. Heinemeyer, W. Hollik, P. Slavich and G. Weiglein, Towards high precision predictions for the MSSM Higgs sector, Eur. Phys. J. C 28 (2003) 133 [hep-ph/0212020] [INSPIRE].

[63] M. Frank, T. Hahn, S. Heinemeyer, W. Hollik, H. Rzehak and G. Weiglein, The Higgs boson masses and mixings of the complex MSSM in the Feynman-diagrammatic approach, JHEP 02 (2007) 047 [hep-ph/0611326] [INSPIRE].

[64] T. Hahn, S. Heinemeyer, W. Hollik, H. Rzehak and G. Weiglein, High-precision predictions for the light CP-even Higgs boson mass of the minimal supersymmetric standard model, Phys. Rev. Lett. 112 (2014) 141801 [arXiv:1312.4937] [INSPIRE].

[65] S. Borowka, T. Hahn, S. Heinemeyer, G. Heinrich and W. Hollik, Momentum-dependent two-loop QCD corrections to the neutral Higgs-boson masses in the MSSM, Eur. Phys. J. C 74 (2014) 2994 [arXiv: 1404.7074] [INSPIRE].

[66] W. Hollik and S. Paßehr, Higgs boson masses and mixings in the complex MSSM with two-loop top-Yukawa-coupling corrections, JHEP 10 (2014) 171 [arXiv:1409.1687] [INSPIRE].

[67] W. Hollik and S. Paßehr, Two-loop top-Yukawa-coupling corrections to the charged Higgs-boson mass in the MSSM, Eur. Phys. J. C 75 (2015) 336 [arXiv:1502.02394] [INSPIRE].

[68] ATLAS and CMS collaborations, Combined measurement of the Higgs boson mass in pp collisions at $\sqrt{s}=7$ and 8 TeV with the ATLAS and CMS experiments, Phys. Rev. Lett. 114 (2015) 191803 [arXiv:1503.07589] [INSPIRE].

[69] J.A. Casas, A. Lleyda and C. Muñoz, Strong constraints on the parameter space of the MSSM from charge and color breaking minima, Nucl. Phys. B 471 (1996) 3 [hep-ph/9507294] [INSPIRE].

[70] S.R. Coleman, The fate of the false vacuum. 1. Semiclassical theory, Phys. Rev. D 15 (1977) 2929 [Erratum ibid. D 16 (1977) 1248] [INSPIRE].

[71] C.G. Callan Jr. and S.R. Coleman, The fate of the false vacuum. 2. First quantum corrections, Phys. Rev. D 16 (1977) 1762 [INSPIRE].

[72] U. Sarid, Tools for tunneling, Phys. Rev. D 58 (1998) 085017 [hep-ph/9804308] [InSPIRE].

[73] Particle Data Group collaboration, K.A. Olive et al., Review of particle physics, Chin. Phys. C 38 (2014) 090001 [InSPIRE].

[74] N. Arkani-Hamed and S. Dimopoulos, Supersymmetric unification without low energy supersymmetry and signatures for fine-tuning at the LHC, JHEP 06 (2005) 073 [hep-th/0405159] [INSPIRE]. 
[75] G.F. Giudice and A. Romanino, Split supersymmetry, Nucl. Phys. B 699 (2004) 65 [Erratum ibid. B 706 (2005) 487] [hep-ph/0406088] [INSPIRE].

[76] Workshop "Precision SUSY Higgs Mass Calculation Initiative" webpage, https://sites.google.com/site/kutsmh/home, Heidelberg Germany January 20-22 2016.

[77] G. 't Hooft and M.J.G. Veltman, Scalar one loop integrals, Nucl. Phys. B 153 (1979) 365 [INSPIRE].

[78] G. Passarino and M.J.G. Veltman, One loop corrections for $e^{+} e^{-}$annihilation into $\mu^{+} \mu^{-}$in the Weinberg model, Nucl. Phys. B 160 (1979) 151 [INSPIRE].

[79] J.A. Casas and S. Dimopoulos, Stability bounds on flavor violating trilinear soft terms in the MSSM, Phys. Lett. B 387 (1996) 107 [hep-ph/9606237] [INSPIRE].

[80] T. Golling et al., Physics at a $100 \mathrm{TeV}$ pp collider: beyond the standard model phenomena, arXiv: 1606.00947 [INSPIRE].

[81] CEPC-SPPC Study Group collaboration, CEPC-SPPC preliminary conceptual design report. 2. Accelerator, IHEP-CEPC-DR-2015-01, (2015) [IHEP-TH-2015-01] [HEP-EP-2015-01] [INSPIRE]. 